\title{
ON THE KATO PROBLEM AND EXTENSIONS FOR DEGENERATE ELLIPTIC OPERATORS
}

\author{
DAVID CRUZ-URIBE, OFS, JOSÉ MARÍA MARTELL, AND CRISTIAN RIOS
}

\begin{abstract}
We study the Kato problem for divergence form operators whose ellipticity may be degenerate. The study of the Kato conjecture for degenerate elliptic equations was begun in $[16,17,19]$. In these papers the authors proved that given an operator $L_{w}=-w^{-1} \operatorname{div}(A \nabla)$, where $w$ is in the Muckenhoupt class $A_{2}$ and $A$ is a $w$-degenerate elliptic measure (that is, $A=w B$ with $B(x)$ an $n \times n$ bounded, complex-valued, uniformly elliptic matrix), then $L_{w}$ satisfies the weighted estimate $\left\|\sqrt{L_{w}} f\right\|_{L^{2}(w)} \approx\|\nabla f\|_{L^{2}(w)}$. In the present paper we solve the $L^{2}$-Kato problem for a family of degenerate elliptic operators. We prove that under some additional conditions on the weight $w$, the following unweighted $L^{2}$-Kato estimates hold:

$$
\left\|L_{w}^{1 / 2} f\right\|_{L^{2}\left(\mathbb{R}^{n}\right)} \approx\|\nabla f\|_{L^{2}\left(\mathbb{R}^{n}\right)} .
$$

This extends the celebrated solution to the Kato conjecture by Auscher, Hofmann, Lacey, McIntosh, and Tchamitchian, allowing the differential operator to have some degree of degeneracy in its ellipticity. For example, we consider the family of operators $L_{\gamma}=-|x|^{\gamma} \operatorname{div}\left(|x|^{-\gamma} B(x) \nabla\right)$, where $B$ is any bounded, complex-valued, uniformly elliptic matrix. We prove that there exists $\epsilon>0$, depending only on dimension and the ellipticity constants, such that

$$
\left\|L_{\gamma}^{1 / 2} f\right\|_{L^{2}\left(\mathbb{R}^{n}\right)} \approx\|\nabla f\|_{L^{2}\left(\mathbb{R}^{n}\right)}, \quad-\epsilon<\gamma<\frac{2 n}{n+2} .
$$

The case $\gamma=0$ corresponds to the case of uniformly elliptic matrices. Hence, our result gives a range of $\gamma$ 's for which the classical Kato square root proved in [4] is an interior point.

Our main results are obtained as a consequence of a rich Calderón-Zygmund theory developed for certain operators naturally associated with $L_{w}$. These results, which are of independent interest, establish estimates on $L^{p}(w)$, and also on $L^{p}(v d w)$ with $v \in A_{\infty}(w)$, for the associated semigroup, its gradient, the functional calculus, the Riesz transform, and vertical square functions. As an application, we solve some unweighted $L^{2}$-Dirichlet, Regularity and Neumann boundary value problems for degenerate elliptic operators.
\end{abstract}

Date: October 6, 2016. Revised: June 7, 2017.

2010 Mathematics Subject Classification. 35B45, 35J15, 35J25, 35J70, 42B20, 42B37, 47A07, 47B44, 47D06.

Key words and phrases. Muckenhoupt weights, degenerate elliptic operators, Kato problem, semigroups, holomorphic functional calculus, square functions, square roots of elliptic operators, Riesz transforms, Dirichlet problem, regularity problem, and Neumann problem.

The first author is supported by NSF grant 1362425 and research funds provided by the Dean of Arts \& Sciences at the University of Alabama. While substantial portions of this work was done he was supported by the Stewart-Dorwart faculty development fund at Trinity College. The second authors acknowledges financial support from the Spanish Ministry of Economy and Competitiveness, through the "Severo Ochoa Programme for Centres of Excellence in R\&D" (SEV-2015-0554). He also acknowledges that the research leading to these results has received funding from the European Research Council under the European Union's Seventh Framework Programme (FP7/2007-2013)/ ERC agreement no. 615112 HAPDEGMT. The third author is supported by the Natural Sciences and Engineering Research Council of Canada Discovery Grant RT733901. The authors warmly thank P. Auscher and S. Hofmann for several useful discussions and their many suggestions. 


\section{Contents}

1. Introduction 2

2. Preliminaries $\quad 5$

Weights $\quad 5$

$\begin{array}{ll}\text { Degenerate elliptic operators } & 7\end{array}$

Holomorphic functional calculus $\quad 8$

Gaffney-type estimates $\quad 9$

The Kato estimate $\quad 10$

Off-diagonal estimates $\quad 11$

3. Off-diagonal estimates for the semigroup $e^{-t L_{w}} \quad 13$

4. The functional calculus 16

5. Square function estimates for the semigroup 19

6. Reverse inequalities 24

7. The gradient of the semigroup $\sqrt{t} \nabla e^{-t L_{w}} \quad 29$

8. An upper bound for $\mathcal{K}\left(L_{w}\right) \quad 33$

A reverse Hölder inequality 33

The Hodge projection 34

Boundedness of the Riesz transform 34

Boundedness of the gradient of the semigroup 35

9. Riesz transform estimates 35

10. Square function estimates for the gradient of the semigroup 40

11. Unweighted $L^{2}$ Kato estimates 42

12. Applications to $L^{2}$ boundary value problems 47

References $\quad 49$

\section{InTRODUCTION}

We study the degenerate elliptic operators $L_{w}=-w^{-1} \operatorname{div} A \nabla$, where $w$ is in the Muckenhoupt class $A_{2}$ and $A(x)$ is an $n \times n$ complex-valued matrix that satisfies the degenerate ellipticity condition

$$
\lambda w(x)|\xi|^{2} \leq \operatorname{Re}\langle A(x) \xi, \xi\rangle, \quad|\langle A(x) \xi, \eta\rangle| \leq \Lambda w(x)|\xi||\eta|, \quad \xi, \eta \in \mathbb{C}^{n}, \text { a.e. } x \in \mathbb{R}^{n} .
$$

Equivalently, $A(x)=w(x) B(x)$, where $B$ is an $n \times n$ complex-valued matrix that satisfies the uniform ellipticity conditions

$$
\lambda|\xi|^{2} \leq \operatorname{Re}\langle B(x) \xi, \xi\rangle, \quad|\langle B(x) \xi, \eta\rangle| \leq \Lambda|\xi||\eta|, \quad \xi, \eta \in \mathbb{C}^{n} \text {, a.e. } x \in \mathbb{R}^{n} .
$$

Such operators were first studied (with $A$ a real symmetric matrix) by Fabes, Kenig and Serapioni [21]. When $A$ is complex-valued and uniformly elliptic (i.e. $w \equiv 1$ ), a landmark result was the proof of the Kato conjecture by Auscher, Hofmann, Lacey, McIntosh, and Tchamitchian [4]: that for all $f \in H^{1}$,

$$
\left\|L^{1 / 2} f\right\|_{2} \approx\|\nabla f\|_{2}
$$

The proof of this long-standing conjecture led naturally to the study of the operators associated with $L$ : the semigroup $e^{-t L}$, its gradient $\sqrt{t} \nabla e^{-t L}$, the Riesz transform $\nabla L^{-1 / 2}$, the $H^{\infty}$ functional calculus and square functions: for details and complete references, see Auscher [2]. These estimates are interesting in themselves; moreover, it is well known that $L^{p}$ estimates 
for these operators yield regularity results for boundary value problems for $L$ : for details, see the introduction to [10].

In [19] (see also [16, 17, 9]) the first and third authors solved the Kato problem for degenerate elliptic operators: they showed that if $w \in A_{2}$ and $A$ satisfies the degenerate ellipticity conditions, then for all $f \in H^{1}(w)$,

$$
\left\|L_{w}^{1 / 2} f\right\|_{L^{2}(w)} \approx\|\nabla f\|_{L^{2}(w)} .
$$

In this paper we consider the problem of determining those $A_{2}$ weights such that the classical Kato problem can be solved for $L_{w}$ : that is, finding weights such that $L_{w}$ satisfies the unweighted estimate

$$
\left\|L_{w}^{1 / 2} f\right\|_{L^{2}\left(\mathbb{R}^{n}\right)} \approx\|\nabla f\|_{L^{2}\left(\mathbb{R}^{n}\right)}
$$

for $f$ in a class of nice functions (a posteriori, by standard density arguments, the estimate can be extended to all $\left.f \in H^{1}\left(\mathbb{R}^{n}\right)\right)$. We solve this problem in two steps. The first is to prove weighted $L^{p}$ estimates for some operators associated with $L_{w}$ (the semigroup, its gradient, the Riesz transform, the functional calculus, and square functions.) These results, which are of interest in their own right, are analogous to those obtained in the uniformly elliptic case. However, a significant technical obstruction is that given a weight $w \in A_{2}$, while it is the case that there exists $\epsilon>0$ such that $w \in A_{2-\epsilon}$, it is easy to construct examples to show that $\epsilon$ may be arbitrarily small. Therefore, our bounds in the range $1<p<2$ need to take this into account.

The second step is to find conditions on the weight $w$ so that these operators satisfy unweighted $L^{2}$ estimates. Both steps are carried out simultaneously, and the proofs are intertwined. Our approach is to apply the theory of off-diagonal estimates on balls developed by Auscher and the second author $[5,6,7,8]$. We will in fact prove weighted estimates on $L^{p}(v d w)$, where $v$ satisfies Muckenhoupt and reverse Hölder conditions with respect to the measure $d w=w d x: L^{p}(w)$ estimates are then obtained by taking $v=1$, and unweighted estimates by taking $v=w^{-1}$.

The unweighted $L^{2}$ estimates are delicate, since they require a careful estimate of the constants that appear. Nevertheless, we are able to give useful sufficient conditions: e.g., $w \in A_{1} \cap R H_{\frac{n}{2}+1}$. (For definitions of these classes, see Section 2 below.) For example, we have the following result that is a special case of one of our main results (cf. Theorem 11.11).

Theorem 1.2. Let $L_{w}=-w^{-1} \operatorname{div} A \nabla$ be a degenerate elliptic operator as above. If $w \in$ $A_{1} \cap R H_{\frac{n}{2}+1}$, then the Kato problem can be solved for $L_{w}$ : for every $f \in H^{1}\left(\mathbb{R}^{n}\right)$,

$$
\left\|L_{w}^{1 / 2} f\right\|_{L^{2}\left(\mathbb{R}^{n}\right)} \approx\|\nabla f\|_{L^{2}\left(\mathbb{R}^{n}\right)}
$$

The implicit constants depend only on the dimension, the ellipticity constants, and the $A_{1}$ and $R H_{\frac{n}{2}+1}$ constants of $w$.

Furthermore, if we define $L_{\gamma}=-|x|^{\gamma} \operatorname{div}\left(|x|^{-\gamma} B(x) \nabla\right)$, where $B$ is an $n \times n$ complexvalued matrix that satisfies the uniform ellipticity condition, then there exists $0<\epsilon<\frac{1}{2}$ small enough (depending only on the dimension and the ratio $\Lambda / \lambda$ ) such that

$$
\left\|L_{\gamma}^{1 / 2} f\right\|_{L^{2}\left(\mathbb{R}^{n}\right)} \approx\|\nabla f\|_{L^{2}\left(\mathbb{R}^{n}\right)}, \quad-\epsilon<\gamma<\frac{2 n}{n+2}
$$

Remark 1.3. In Theorem 1.2 the operator $L_{w}^{1 / 2}$ is a priori only defined on $H^{1}(w)$; however this means that it is defined on $C_{0}^{\infty}\left(\mathbb{R}^{n}\right)$ and so by a standard density argument we can 
extend our results to all $f \in H^{1}\left(\mathbb{R}^{n}\right)$. Hereafter we will make this extension without further comment.

We emphasize that in Theorem 1.2, when $\gamma=0$ we are back at the uniformly elliptic case, which is the celebrated solution to the Kato square root problem by Auscher, Hofmann, Lacey, McIntosh, and Tchamitchian in [4]. Here we are able to find a range of $\gamma$ 's for which the same estimates hold and the classical Kato square root problem (i.e., $\gamma=0$ ) is an interior point in that range.

These unweighted $L^{2}$ estimates have important applications to boundary value problems for degenerate elliptic operators. Consider, for example, the following Dirichlet problem on $\mathbb{R}_{+}^{n+1}=\mathbb{R}^{n} \times[0, \infty)$ :

$$
\begin{cases}\partial_{t}^{2} u-L_{w} u=0, & \text { on } \mathbb{R}_{+}^{n+1} \\ u=f & \text { on } \partial \mathbb{R}_{+}^{n+1}=\mathbb{R}^{n}\end{cases}
$$

If $f \in L^{2}\left(\mathbb{R}^{n}\right)$, then $u(x, t)=e^{-t L_{w}^{1 / 2}} f(x)$ is a solution, and if $L_{w}$ has a bounded $H^{\infty}$ functional calculus on $L^{2}$, then $\sup _{t>0}\|u(\cdot, t)\|_{2} \lesssim\|f\|_{2}$. Similar results hold for the corresponding Neumann and Regularity problems.

Our proofs are unavoidably technical, and the results for each operator considered build upon what was proved previously for other operators. We have organized the material as follows. In Section 2 we gather some essential definitions and results about weights, degenerate elliptic operators, and off-diagonal estimates. Central to all of our subsequent work are Theorems 2.35 and 2.39 (which were proved in [5]).

In Sections 3, 4, and 5 we prove estimates for the semigroup $e^{-t L_{w}}, t>0$, the $H^{\infty}$ functional calculus (i.e., operators $\varphi\left(L_{w}\right)$ where $\left.\varphi \in \mathcal{H}^{\infty}\right)$, the vertical square function associated to the semigroup,

$$
g_{L_{w}} f(x)=\left(\int_{0}^{\infty}\left|\left(t L_{w}\right)^{1 / 2} e^{-t L_{w}} f(x)\right|^{2} \frac{d t}{t}\right)^{1 / 2},
$$

and its discrete analog. Here and in subsequent sections we prove both $L^{p}(w)$ estimates and weighted $L^{p}(v d w)$ estimates. In many cases these results are proved simultaneously, with the unweighted results (i.e., in $\left.L^{p}(w)\right)$ following from the weighted results (i.e., in $L^{p}(v d w)$ ) by taking $v=1$.

In Section 6 we prove the so-called reverse inequality, $\left\|L_{w}^{1 / 2}\right\|_{L^{p}(w)} \lesssim\|\nabla f\|_{L^{p}(w)}$, that generalizes the $L^{2}(w)$ estimate in (1.1). We note that while the equivalence in (1.1) follows at once from the reverse inequality for $p=2$ by duality, the two inequalities behave differently when $p \neq 2$.

In Sections 7 and 8 we prove estimates for the gradient of the semigroup, $\sqrt{t} \nabla e^{-t L_{w}}$. The proof that there exists $q_{+}>2$ such that this operator satisfies $L^{p}(w)$ estimates for $2<p<q_{+}$is quite involved as it requires preliminary estimates for the Riesz transform and the Hodge projection. We note that, as opposed to the non-degenerate case, here we cannot use "global" embeddings, nor can we rescale. Also we cannot expect to obtain that the gradient of the semigroup maps globally $L^{2}(w)$ into $L^{p}(w)$ for $p \neq 2$. All these difficulties arise naturally from the lack of isotropy of the natural underlying measure $w(x) d x$ and make the typical arguments used in the uniformly elliptic case (cf. [2, Chapter 4]) unusable. We also note that in some sense our result is the best possible: even in the non-degenerate 
case it is known [2] that given any $p>2$ there exists a matrix $A$ and operator $L$ such that gradient of the semigroup is not bounded on $L^{p}$.

In Section 9 we prove $L^{p}(w)$ estimates for the Riesz transform $\nabla L^{-1 / 2}$, and in Section 10 we prove $L^{p}(w)$ estimates for the square function associated to the gradient of the semigroup,

$$
G_{L_{w}} f(x)=\left(\int_{0}^{\infty}\left|t^{1 / 2} \nabla e^{-t L_{w}} f(x)\right|^{2} \frac{d t}{t}\right)^{1 / 2} .
$$

In Section 11 we prove unweighted $L^{2}$ inequalities for the operators we have considered in previous sections. These are a consequence of the weighted estimates and are obtained by taking $v=w^{-1}$. The main problem is determining conditions on $w$ for these to hold. We essentially have two different kinds of estimates: one for operators that do not involve the gradient, and one for those that do. The latter are more delicate as they involve careful bounds for the parameter $q_{+}$from Section 8 in terms of the weight $w$. We also show that we get unweighted $L^{p}$ estimates for $p$ very close to 2 .

Finally, in Section 12 we describe in more detail the application of our results to $L^{2}$ boundary value problems for degenerate elliptic operators. The results in this section our the culmination of our work as they depend on all the estimates derived in previous sections.

As we were completing this project, we learned that related results had been obtained independently by other authors. In [26] Le studied (among other things) the $L^{p}(w)$ theory for some of the operators considered here and proved estimates for values of $p$ in the range $(2-\epsilon, 2+\epsilon)$. His proofs differ from ours in a number of details. In [24] Hofmann, Le and Morris established some Carleson measure estimates and considered the Dirichlet problem for degenerate elliptic operators. Also, very recently we learned that Yang and Zhang [30] proved Kato type estimates in $L^{p}(w)$ for $p$ in the range $\left(p_{0}, 2\right]$. Finally, we note that the paper [13] complements our work here as it considers the conical square functions associated to the operator $L_{w}$.

\section{Preliminaries}

Throughout $n$ will denote the dimension of the underlying space $\mathbb{R}^{n}$ and we will always assume $n \geq 2$. If we write $A \lesssim B$ we mean that there exists a constant $C$ such that $A \leq C B$. We write $A \approx B$ if $A \lesssim B$ and $B \lesssim A$. The constant $C$ in these estimates may depend on the dimension $n$ and other (fixed) parameters that should be clear from the context. All constants, explicit or implicit, may change at each appearance.

Given a ball $B$, let $r(B)$ denote the radius of $B$. Let $\lambda B$ denote the concentric ball with radius $r(\lambda B)=\lambda r(B)$.

Weights. By a weight $w$ we mean a non-negative, locally integrable function. For brevity, we will often write $d w$ for $w d x$. We will use the following notation for averages: given a set $E$ such that $0<w(E)<\infty$,

$$
f_{E} f d w=\frac{1}{w(E)} \int_{E} f d w
$$

or, if $0<|E|<\infty$,

$$
f_{E} f d x=\frac{1}{|E|} \int_{E} f d x
$$


We state some definitions and basic properties of Muckenhoupt weights. For further details, see $[20,22]$. We say that $w \in A_{p}, 1<p<\infty$, if

$$
[w]_{A_{p}}=\sup _{Q} f_{Q} w(x) d x\left(f_{Q} w(x)^{1-p^{\prime}} d x\right)^{p-1}<\infty .
$$

When $p=1$, we say that $w \in A_{1}$ if

$$
[w]_{A_{1}}=\sup _{Q} f_{Q} w(x) d x \underset{x \in Q}{\operatorname{ess} \sup } w(x)^{-1}<\infty .
$$

We say that $w \in R H_{s}, 1<s<\infty$, if

$$
[w]_{R H_{s}}=\sup _{Q}\left(f_{Q} w(x) d x\right)^{-1}\left(f_{Q} w(x)^{s} d x\right)^{1 / s}<\infty,
$$

and we say that $w \in R H_{\infty}$ if

$$
[w]_{R H_{\infty}}=\sup _{Q}\left(f_{Q} w(x) d x\right)^{-1} \operatorname{ess} \sup _{x \in Q} w(x)<\infty .
$$

Let

$$
A_{\infty}=\bigcup_{1 \leq p<\infty} A_{p}=\bigcup_{1<s \leq \infty} R H_{s}
$$

Weights in the $A_{p}$ and $R H_{s}$ classes have a self-improving property: if $w \in A_{p}$, there exists $\epsilon>0$ such that $w \in A_{p-\epsilon}$, and similarly if $w \in R H_{s}$, then $w \in R H_{s+\delta}$ for some $\delta>0$. Hereafter, given $w \in A_{p}$, let

$$
r_{w}=\inf \left\{p: w \in A_{p}\right\}, \quad s_{w}=\sup \left\{q: w \in R H_{q}\right\} .
$$

An important property of $A_{p}$ weights is that they are doubling: given $w \in A_{p}$, for all $\tau \geq 1$ and any ball $B$,

$$
w(\tau B) \leq[w]_{A_{p}} \tau^{p n} w(B) .
$$

In particular, hereafter let $D \leq p n$ be the doubling order of $w$ : that is the smallest exponent such that this inequality holds.

As a consequence of this doubling property, we have that with the ordinary Euclidean distance $|\cdot|,\left(\mathbb{R}^{n}, d w,|\cdot|\right)$ is a space of homogeneous type. In this setting we can define the new weight classes $A_{p}(w)$ and $R H_{s}(w)$ by replacing Lebesgue measure in the definitions above with $d w$ : e.g., $v \in A_{p}(w)$ if

$$
[v]_{A_{p}(w)}=\sup _{Q} f_{Q} v(x) d w\left(f_{Q} v(x)^{1-p^{\prime}} d w\right)^{p-1}<\infty .
$$

It follows at once from these definitions that there is a "duality" relationship between the weighted and unweighted $A_{p}$ and $R H_{s}$ conditions: $v=w^{-1} \in A_{p}(w)$ if and only if $w \in R H_{p^{\prime}}$ and $v=w^{-1} \in R H_{s}(w)$ if and only if $w \in A_{s^{\prime}}$.

Weighted Poincaré-Sobolev inequalities were proved in [21].

Theorem 2.1. Given $w \in A_{p}, p \geq 1$, let $p_{w}^{*}=\frac{p n r_{w}}{n r_{w}-p}$ if $p<n r_{w}$ and $p_{w}^{*}=\infty$ otherwise. Then for every $p \leq q<p_{w}^{*}$, ball $B$ and $f \in C_{0}^{\infty}(B)$,

$$
\left(f_{B}|f(x)|^{q} d w(x)\right)^{1 / q} \leq C r(B)\left(f_{B}|\nabla f(x)|^{p} d w\right)^{1 / p} .
$$


Moreover, if $f \in C^{\infty}(B)$, then

$$
\left(f_{B}\left|f(x)-f_{B, w}\right|^{q} d w(x)\right)^{1 / q} \leq C r(B)\left(f_{B}|\nabla f(x)|^{p} d w\right)^{1 / p},
$$

where $f_{B, w}=f_{B} f d w$.

Remark 2.4. In the special case when $w \in A_{1}$ and $1<p<n$ we can also take $q=p_{w}^{*}=p^{*}$, i.e., the regular Sobolev exponent. See Pérez [29, Theorem 2.5.2].

Remark 2.5. If we let $q=\frac{n p}{n-1}<p_{w}^{*}$, then we can get a sharp estimate for the constant $C$ in (2.2) and (2.3): it is of the form $C(p, n)[w]_{A_{p}}^{\kappa}$ where $\kappa=\frac{n p-1}{n p(p-1)}$. This follows from the sharp weighted estimates for the fractional integral operator due to Alberico, Cianchi and Sbordone [1] and the standard pointwise estimates used to prove Poincaré-Sobolev inequalities; see [21] for details.

Remark 2.6. By a standard density argument, once we know that (2.3) holds for smooth functions in $B$ we can easily extend that estimate to any function $f \in L^{q}(w)$ with $\nabla f \in$ $L^{p}(w)$. Details are left to the reader.

Degenerate elliptic operators. Given $w \in A_{2}$ and constants $0<\lambda \leq \Lambda<\infty$, let $\mathcal{E}_{n}(w, \lambda, \Lambda)$ denote the class of $n \times n$ matrices $A=\left(A_{i j}(x)\right)_{i, j=1}^{n}$ of complex-valued, measurable functions satisfying the degenerate ellipticity condition

$$
\lambda w(x)|\xi|^{2} \leq \operatorname{Re}\langle A \xi, \xi\rangle, \quad|\langle\mathcal{A} \xi, \eta\rangle| \leq \Lambda w(x)|\xi||\eta|, \quad \xi, \eta \in \mathbb{C}^{n} .
$$

Given $A \in \mathcal{E}_{n}(w, \lambda, \Lambda)$, we define the degenerate elliptic operator in divergence form

$$
L_{w}=-w^{-1} \operatorname{div} A \nabla .
$$

These operators were developed in [16] and we refer the reader there for complete details. Here we sketch the key ideas.

Given a weight $w \in A_{2}$, the space $H^{1}(w)$ is the weighted Sobolev space that is the completion of $C_{c}^{\infty}$ with respect to the norm

$$
\|f\|_{H^{1}(w)}=\left(\int_{\mathbb{R}^{n}}\left(|f(x)|^{2}+|\nabla f(x)|^{2}\right) d w\right)^{1 / 2} .
$$

Note that the space defined above would usually be denoted by $H_{0}^{1}(w)$. The space $H^{1}(w)$ is defined as the set of distributions for which both $f$ and $|\nabla f|$ belong to $L^{2}(w)$. However, since the underlying domain is $\mathbb{R}^{n}$ this definition implies that the "boundary" values vanish in the $L^{2}(w)$-sense, and both definitions agree [28].

Given a matrix $A \in \mathcal{E}_{n}(w, \lambda, \Lambda)$, define $\mathfrak{a}(f, g)$ to be the sesquilinear form

$$
\mathfrak{a}(f, g)=\int_{\mathbb{R}^{n}} A(x) \nabla f(x) \cdot \overline{\nabla g(x)} d x .
$$

Since $w \in A_{2}$ and $A$ satisfies (2.7), $\mathfrak{a}$ is a closed, maximally accretive, continuous sesquilinear form. Therefore, there exists an operator $L_{w}$ whose domain $\mathcal{D}\left(L_{w}\right) \subset H^{1}(w)$ is dense in $L^{2}(w)$ and such that for every $f \in \mathcal{D}\left(L_{w}\right)$ and every $g \in H^{1}(w)$,

$$
\mathfrak{a}(f, g)=\left\langle L_{w} f, g\right\rangle_{w}=\int_{\mathbb{R}^{n}} L_{w} f(x) \overline{g(x)} d w .
$$


We note that the operator $L_{w}$ is one to one. Indeed, if $u, v \in \mathcal{D}\left(L_{w}\right)$ are such that $L_{w} u=L_{w} v$, then for all $g \in H^{1}(w)$

$$
0=\int_{\mathbb{R}^{n}} A(x) \nabla(u(x)-v(x)) \cdot \overline{\nabla g(x)} d x .
$$

Taking $g=u-v$ implies $\nabla u(x)=\nabla v(x)$ and so $u=v$.

The properties of the sesquilinear form guarantee that on $L^{2}(w)$ there exists a bounded, strongly continuous semigroup $e^{-t L_{w}}$. Further, it has a holomorphic extension. Let

$$
\Sigma_{\omega}=\{z \in \mathbb{C}: z \neq 0,|\arg (z)|<\omega\}
$$

and define $\vartheta, \vartheta^{*} \in[0, \pi / 2)$ by

$$
\vartheta=\sup \left\{\left|\arg \langle L f, f\rangle_{w}\right|: f \in \mathcal{D}\left(L_{w}\right)\right\}, \quad \vartheta^{*}=\arctan \sqrt{\frac{\Lambda^{2}}{\lambda^{2}}-1} .
$$

Then there exists a complex semigroup $e^{-z L_{w}}$ on $\Sigma_{\pi / 2-\vartheta}$ of bounded operators on $L^{2}(w)$. By the weighted ellipticity condition (2.7), we have that $0 \leq \vartheta \leq \vartheta^{*}<\pi / 2$.

Holomorphic functional calculus. Our operator $L_{w}$ is "an operator of type $\omega$ " with $\omega=\vartheta$, as defined in [27]. Indeed, the ellipticity conditions imply that $L_{w}$ is closed and densely defined, its spectrum is contained in $\Sigma_{\vartheta}$, and its resolvent satisfies standard decay estimates [16]. Therefore, we can define an $L^{2}(w)$ functional calculus as in [27].

Given $\mu \in(\vartheta, \pi)$, let $\mathcal{H}^{\infty}\left(\Sigma_{\mu}\right)$ be the collection of bounded holomorphic functions on $\Sigma_{\mu}$. To define $\varphi\left(L_{w}\right)$ for $\varphi \in \mathcal{H}^{\infty}\left(\Sigma_{\mu}\right)$ we first consider a smaller class: we say that $\varphi \in \mathcal{H}_{0}^{\infty}\left(\Sigma_{\mu}\right)$ if for some $c, s>0$ it satisfies

$$
|\varphi(z)| \leq c|z|^{s}(1+|z|)^{-2 s}, \quad z \in \Sigma_{\mu}
$$

We then have an integral representation of $\varphi\left(L_{w}\right)$. Let $\Gamma_{\theta}$ be the boundary of $\Sigma_{\theta}$ with positive orientation, and let $\vartheta<\theta<\nu<\min (\mu, \pi / 2)$; then

$$
\varphi\left(L_{w}\right)=\int_{\Gamma_{\pi / 2-\theta}} e^{-z L_{w}} \eta(z) d z
$$

where

$$
\eta(z)=\frac{1}{2 \pi i} \int_{\gamma_{\nu}(z)} e^{\zeta z} \varphi(\zeta) d \zeta
$$

and $\gamma_{\nu}(z)=\mathbb{R}^{+} e^{i \operatorname{sign}(\operatorname{Im}(z)) \nu}$. Note that

$$
|\eta(z)| \lesssim \min \left(1,|z|^{-s-1}\right), \quad z \in \Gamma_{\pi / 2-\theta},
$$

so the representation $(2.10)$ converges in $L^{2}(w)$, and we have the bound

$$
\left\|\varphi\left(L_{w}\right) f\right\|_{L^{2}(w)} \leq C\|\varphi\|_{\infty}\|f\|_{L^{2}(w)}, \quad f \in \mathcal{H}_{0}^{\infty}\left(\Sigma_{\mu}\right) .
$$

Now, since $L_{w}$ is a one-to-one operator of type $\omega$, it has dense range [14, Theorem 2.3], and so the results in [27] (see also [14, Corollary 2.2]) imply that $L_{w}$ has an $H^{\infty}$ functional calculus and (2.12) extends to all of $\mathcal{H}^{\infty}\left(\Sigma_{\mu}\right)$. Moreover, in [27, Section 8] the equivalence between the existence of this $H^{\infty}$ functional calculus and square function estimates for $L_{w}$ and $L_{w}^{*}$ is established:

$$
\left\{\int_{0}^{\infty}\left\|\varphi\left(t L_{w}\right)\right\|_{L^{2}(w)}^{2} \frac{d t}{t}\right\}^{\frac{1}{2}} \leq C\|\varphi\|_{\infty}\|f\|_{L^{2}(w)}, \quad \varphi \in \mathcal{H}_{0}^{\infty}\left(\Sigma_{\mu}\right),
$$


with similar estimates for $L_{w}^{*}$.

The operators $\varphi\left(L_{w}\right)$ also have the following properties:

- If $\varphi$ and $\psi$ are bounded holomorphic functions, then we have the operator identity $\varphi(L) \psi(L)=(\varphi \psi)(L)$.

- Given any sequence $\left\{\varphi_{k}\right\}$ of bounded holomorphic functions converging uniformly on compact subsets of $\Sigma_{\mu}$ to $\varphi$, we have that $\varphi_{k}\left(L_{w}\right)$ converges to $\varphi\left(L_{w}\right)$ in the strong operator topology (of operators on $L^{2}(w)$ ).

Remark 2.14. The $H^{\infty}$ functional calculus can be extended to more general holomorphic functions, such as powers, for which the operators $\varphi\left(L_{w}\right)$ can be defined as unbounded operators: see [23, 27].

Gaffney-type estimates. The semigroup and its gradient satisfy Gaffney-type estimates on $L^{2}(w)$. Below, we will see that these are a particular case of what we will call full off-diagonal estimates: see Definition 2.33.

Theorem 2.15. Given $w \in A_{2}$ and $A \in \mathcal{E}_{n}(w, \lambda, \Lambda)$, for any closed sets $E$ and $F$, for $f \in L^{2}(w)$ and for all $z \in \Sigma_{\nu}$, where $0<\nu<\frac{\pi}{2}-\vartheta$,

(2) $\left\|\sqrt{z} \nabla e^{-z L_{w}}\left(f \chi_{E}\right) \chi_{F}\right\|_{L^{2}(w)} \leq C e^{-\frac{c d(E, F)^{2}}{|z|}}\left\|f \chi_{E}\right\|_{L^{2}(w)}$,

(3) $\left\|z L_{w} e^{-z L_{w}}\left(f \chi_{E}\right) \chi_{F}\right\|_{L^{2}(w)} \leq C e^{-\frac{c d(E, F)^{2}}{|z|}}\left\|f \chi_{E}\right\|_{L^{2}(w)}$.

Proof. The semigroup estimate (1) was proved in [16, Theorem 1.6] for real $z$, but the same proof can be readily modified to prove the analytic version. Alternatively, estimates (1) and (2) follow from the resolvent bounds

$$
\left\|\left(1+z^{2} L_{w}\right)^{-1}\left(f \chi_{E}\right) \chi_{F}\right\|_{L^{2}(w)} \leq C e^{-\frac{c d(E, F)}{|z|}}\left\|f \chi_{E}\right\|_{L^{2}(w)},
$$

and

$$
\left\|z \nabla\left(1+z^{2} L_{w}\right)^{-1}\left(f \chi_{E}\right) \chi_{F}\right\|_{L^{2}(w)} \leq C e^{-\frac{c d(E, F)}{|z|}}\left\|f \chi_{E}\right\|_{L^{2}(w)},
$$

obtained in [19, Lemma 2.10] for $z \in \Sigma_{\frac{\pi}{2}+\nu}$, together with the integral representation of the semigroup

$$
e^{-z L_{w}} f=\frac{1}{2 \pi} \int_{\Gamma} e^{z \zeta}\left(\zeta+L_{w}\right)^{-1} f d \zeta
$$

where $\Gamma$ is the boundary of $\Sigma_{\theta}$ with positive orientation and $\frac{\pi}{2}<\theta<\frac{\pi}{2}+\nu-\arg (z)$.

Finally, from (2.16) and (2.17) we obtain the estimate

$$
\left\|z^{2} L_{w}\left(1+z^{2} L_{w}\right)^{-1}\left(f \chi_{E}\right) \chi_{F}\right\|_{L^{2}(w)} \leq C e^{-\frac{c d(E, F)}{|z|}}\left\|f \chi_{E}\right\|_{L^{2}(w)},
$$

and then by the same kind of argument we get (3). 
The Kato estimate. The starting point for all of our estimates is the $L^{2}(w)$ Kato estimates for the square root operator $L_{w}^{1 / 2}$ proved in [19] (see also [9] for a different proof). This operator is the unique, maximal accretive operator such that $L_{w}^{1 / 2} L_{w}^{1 / 2}=L_{w}$. It has the integral representation

$$
L_{w}^{1 / 2}=\frac{1}{\sqrt{\pi}} \int_{0}^{\infty} \sqrt{t} L_{w} e^{-t L_{w}} \frac{d t}{t} .
$$

(For further details, see [10, 27].)

Theorem 2.18. [19, Theorem 1.1] Given $w \in A_{2}$ and $A \in \mathcal{E}_{n}(w, \lambda, \Lambda)$, the domain of $L_{w}$ is $H^{1}(w)$ and there exist constants $c$ and $C$, depending on $n, \Lambda / \lambda$ and $[w]_{A_{2}}$, such that for all $f \in H^{1}(w)$,

$$
c\|\nabla f\|_{L^{2}(w)} \leq\left\|L_{w}^{1 / 2} f\right\|_{L^{2}(w)} \leq C\|\nabla f\|_{L^{2}(w)}
$$

The Riesz transform associated to $L_{w}$ is the operator $\nabla L_{w}^{-1 / 2}$. Formally, by (2.19) we have that the Riesz transform is a bounded operator on $L^{2}\left(w, \mathbb{C}^{n}\right)$. To legitimize this, we define

$$
\nabla L_{w}^{-1 / 2}=\frac{1}{\sqrt{\pi}} \int_{0}^{\infty} \sqrt{t} \nabla e^{-t L_{w}} \frac{d t}{t} .
$$

However, it is not immediate that this integral converges at 0 or $\infty$. To rectify this, for $\epsilon>0$ define

$$
S_{\epsilon}=S_{\epsilon}\left(L_{w}\right)=\frac{1}{\sqrt{\pi}} \int_{\epsilon}^{1 / \epsilon} \sqrt{t} e^{-t L_{w}} \frac{d t}{t} .
$$

Since $S_{\epsilon}(z)$ is a uniformly bounded holomorphic function on the right half plane for all $0<\epsilon<1$, by the $L^{2}(w)$ functional calculus described above, $S_{\epsilon}\left(L_{w}\right)$ is uniformly bounded on $L^{2}(w)$ for that range of $\epsilon$. Further, for $f \in L_{c}^{\infty}, S_{\epsilon} f \in \mathcal{D}\left(L_{w}\right) \subset \mathcal{D}\left(L_{w}^{1 / 2}\right)$, and so by inequality (2.19) and the functional calculus,

$$
\left\|\nabla S_{\epsilon} f\right\|_{L^{2}(w)} \lesssim\left\|L^{1 / 2} S_{\epsilon} f\right\|_{L^{2}(w)}=\left\|\varphi_{\epsilon}\left(L_{w}\right) f\right\|_{L^{2}(w)}
$$

where

$$
\varphi_{\epsilon}(z)=\frac{1}{\sqrt{\pi}} \int_{\epsilon}^{1 / \epsilon} \sqrt{t} \sqrt{z} e^{-t z} \frac{d t}{t} .
$$

The sequence $\left\{\varphi_{\epsilon}\right\}$ is uniformly bounded and converges uniformly to 1 on compact subsets of the sector $\Sigma_{\mu}, 0<\mu<\pi / 2$. Therefore, $L^{1 / 2} S_{\epsilon} f \rightarrow f$ strongly in $L^{2}(w)$. If we combine this fact with $(2.22)$ we see that $\left\{\nabla S_{\epsilon} f\right\}$ is Cauchy and so it converges in $L^{2}(w)$. We therefore define

$$
\nabla L^{-1 / 2} f=\lim _{\epsilon \rightarrow 0} \nabla S_{\epsilon} f
$$

where the limit is in $L^{2}(w)$.

Given this definition, hereafter, when we are proving $L^{2}(w)$ estimates for the Riesz transform, we should actually prove estimates for $\nabla S_{\epsilon}$ that are independent of $\epsilon$. These arguments will remain implicit unless there are details we need to emphasize. 
Off-diagonal estimates. Off-diagonal estimates as we define them were introduced in [7] and we will refer repeatedly to this paper for further information and results. Throughout this section we will assume that given a weight $w, w \in A_{2}$.

Given a ball $B$, for $j \geq 2$ we define the annuli $C_{j}(B)=2^{j+1} B \backslash 2^{j} B$. We let $C_{1}(B)=4 B$. By a slight abuse of notation, we will define

$$
f_{C_{j}(B)} h d w=\frac{1}{w\left(2^{j+1} B\right)} \int_{C_{j}(B)} h d w .
$$

If $w \in A_{2}$ (as it will be hereafter), then $w\left(2^{j+1} B\right) \approx w\left(C_{j}(B)\right)$, so this definition is equivalent to the one given above up to a constant. Finally, for $s>0$ we set $\Upsilon(s)=\max \left\{s, s^{-1}\right\}$.

Definition 2.23. Given $1 \leq p \leq q \leq \infty$, a family $\left\{T_{t}\right\}_{t>0}$ of sublinear operators satisfies $L^{p}(w)-L^{q}(w)$ off-diagonal estimates on balls, denoted by

$$
T_{t} \in \mathcal{O}\left(L^{p}(w) \rightarrow L^{q}(w)\right),
$$

if there exist constants $\theta_{1}, \theta_{2}>0$ and $c>0$ such that for every $t>0$ and for any ball $B$, setting $r=r(B)$,

$$
\left(f_{B}\left|T_{t}\left(\chi_{B} f\right)\right|^{q} d w\right)^{\frac{1}{q}} \lesssim \Upsilon\left(\frac{r}{\sqrt{t}}\right)^{\theta_{2}}\left(f_{B}|f|^{p} d w\right)^{\frac{1}{p}}
$$

and for all $j \geq 2$,

$$
\left(f_{B}\left|T_{t}\left(\chi_{C_{j}(B)} f\right)\right|^{q} d w\right)^{\frac{1}{q}} \lesssim 2^{j \theta_{1}} \Upsilon\left(\frac{2^{j} r}{\sqrt{t}}\right)^{\theta_{2}} e^{-\frac{c 4^{j} r^{2}}{t}}\left(f_{C_{j}(B)}|f|^{p} d w\right)^{\frac{1}{p}}
$$

and

$$
\left(f_{C_{j}(B)}\left|T_{t}\left(\chi_{B} f\right)\right|^{q} d w\right)^{\frac{1}{q}} \lesssim 2^{j \theta_{1}} \Upsilon\left(\frac{2^{j} r}{\sqrt{t}}\right)^{\theta_{2}} e^{-\frac{c 4^{j} r^{2}}{t}}\left(f_{B}|f|^{p} d w\right)^{\frac{1}{p}} .
$$

If the family of sublinear operators $\left\{T_{z}\right\}_{z \in \Sigma_{\mu}}$ is defined on a complex sector $\Sigma_{\mu}$, we say that it satisfies $L^{p}(w)-L^{q}(w)$ off-diagonal estimates on balls in $\Sigma_{\mu}$ if (2.24), (2.25) and (2.26) hold for $z \in \Sigma_{\mu}$ with $t$ replaced by $|z|$ in the righthand terms. We denote this by $T_{z} \in \mathcal{O}\left(L^{p}(w) \rightarrow L^{q}(w), \Sigma_{\mu}\right)$.

We give some basic properties of off-diagonal estimates on balls as a series of lemmas taken from [7, Section 2.2]. The first follows immediately by real interpolation, the second by Hölder's inequality, and the third by duality.

Lemma 2.27. Given $1 \leq p_{i} \leq q_{i} \leq \infty, i=1,2$, if $T_{t} \in \mathcal{O}\left(L^{p_{1}}(w) \rightarrow L^{q_{1}}(w)\right)$, and $T_{t}: L^{p_{2}}(w) \rightarrow L^{q_{2}}(w)$ is uniformly bounded, then $T_{t} \in \mathcal{O}\left(L^{p_{\theta}}(w) \rightarrow L^{q_{\theta}}(w)\right), 0<\theta<1$, where

$$
\frac{1}{p_{\theta}}=\frac{\theta}{p_{1}}+\frac{1-\theta}{p_{2}}, \quad \frac{1}{q_{\theta}}=\frac{\theta}{q_{1}}+\frac{1-\theta}{q_{2}} .
$$

Lemma 2.28. If $1 \leq p \leq p_{1} \leq q_{1} \leq q \leq \infty$, then

$$
\mathcal{O}\left(L^{p}(w) \rightarrow L^{q}(w)\right) \subset \mathcal{O}\left(L^{p_{1}}(w) \rightarrow L^{q_{1}}(w)\right) .
$$

Lemma 2.29. If for some $1 \leq p \leq q \leq \infty, T_{t} \in \mathcal{O}\left(L^{p}(w) \rightarrow L^{q}(w)\right)$, and the operators $T_{t}$ are linear, then $T_{t}^{*} \in \mathcal{O}\left(L^{q^{\prime}}(w) \rightarrow L^{p^{\prime}}(w)\right)$. (Here $T_{t}^{*}$ is the dual operator for the inner product $\left.\int_{\mathbb{R}^{n}} f g d w.\right)$ 
Lemma 2.30 ([7, Theorem 2.3]).

(1) If $T_{t} \in \mathcal{O}\left(L^{p}(w) \rightarrow L^{p}(w)\right), 1 \leq p \leq \infty$, then $T_{t}: L^{p}(w) \rightarrow L^{p}(w)$ is uniformly bounded.

(2) If $1 \leq p \leq q \leq r \leq \infty, T_{t} \in \mathcal{O}\left(L^{q}(w) \rightarrow L^{r}(w)\right)$, and $S_{t} \in \mathcal{O}\left(L^{p}(w) \rightarrow L^{q}(w)\right)$, then $T_{t} \circ S_{t} \in \mathcal{O}\left(L^{p}(w) \rightarrow L^{r}(w)\right)$.

Remark 2.31. If $p<q$, then $T_{t} \in \mathcal{O}\left(L^{p}(w) \rightarrow L^{q}(w)\right)$ does not guarantee that $T_{t}$ is bounded from $L^{p}(w)$ to $L^{q}(w)$.

Remark 2.32. Since complex sectors $\Sigma_{\mu}, 0 \leq \mu<\pi$, are closed under addition, the proof of Lemma 2.30 extends to give off-diagonal estimates on complex sectors $\mathcal{O}\left(L^{p}(w) \rightarrow\right.$ $\left.L^{q}(w), \Sigma_{\mu}\right)$.

Definition 2.33. Given $1 \leq p \leq q \leq \infty$, a family of operators $\left\{T_{t}\right\}$ satisfies full off-diagonal estimates from $L^{p}(w)$ to $L^{q}(w)$, denoted by

$$
T_{t} \in \mathcal{F}\left(L^{p}(w) \rightarrow L^{q}(w)\right),
$$

if there exist constants $C, c, \theta>0$ such that given any closed sets $E, F$,

$$
\left\|T_{t}\left(f \chi_{E}\right) \chi_{F}\right\|_{L^{q}(w)} \leq C t^{-\theta} e^{-\frac{c d^{2}(E, F)}{t}}\left\|f \chi_{E}\right\|_{L^{p}(w)} .
$$

The connection between full off-diagonal estimates and off-diagonal estimates on balls is given in the following lemma from [7, Section 3.1].

Lemma 2.34. Given $1 \leq p \leq q \leq \infty$ :

(1) if $T_{t} \in \mathcal{F}\left(L^{p}(w) \rightarrow L^{q}(w)\right)$, then $T_{t}: L^{p}(w) \rightarrow L^{q}(w)$ is uniformly bounded;

(2) $T_{t} \in \mathcal{F}\left(L^{p}(w) \rightarrow L^{p}(w)\right)$ if and only if $T_{t} \in \mathcal{O}\left(L^{p}(w) \rightarrow L^{p}(w)\right)$.

The importance of off-diagonal estimates is that they will let us prove weighted norm inequalities for the operators we are interested in. To do so we will make repeated use of two results first proved in [6]; however, we will use special cases of these results as given in [5, Theorems 2.2 and 2.4].

Theorem 2.35. Given $w \in A_{2}$ and $1 \leq p_{0}<q_{0} \leq \infty$, let $T$ be a sublinear operator acting on $L^{p_{0}}(w),\left\{\mathcal{A}_{r}\right\}_{r>0}$ a family of operators acting from a subspace $\mathcal{D}$ of $L^{p_{0}}(w)$ into $L^{p_{0}}(w)$, and $S$ an operator from $\mathcal{D}$ into the space of measurable functions on $\mathbb{R}^{n}$. Suppose that for every $f \in \mathcal{D}$ and ball $B$ with radius $r$,

$$
\left(f_{B}\left|T\left(I-\mathcal{A}_{r}\right) f\right|^{p_{0}} d w\right)^{1 / p_{0}} \leq \sum_{j \geq 1} g(j)\left(f_{2^{j+1} B}|S f|^{p_{0}} d w\right)^{1 / p_{0}}
$$

and

$$
\left(f_{B}\left|T \mathcal{A}_{r} f\right|^{q_{0}} d w\right)^{1 / q_{0}} \leq \sum_{j \geq 1} g(j)\left(f_{2^{j+1} B}|T f|^{p_{0}} d w\right)^{1 / p_{0}},
$$

where $\sum g(j)<\infty$. Then for every $p, p_{0}<p<q_{0}$, and weights

$$
v \in A_{p / p_{0}}(w) \cap R H_{\left(q_{0} / p\right)^{\prime}}(w)
$$

there is a constant $C$ such that for all $f \in \mathcal{D}$,

$$
\|T f\|_{L^{p}(v d w)} \leq C\|S f\|_{L^{p}(v d w)} .
$$


Remark 2.38. In Theorem 2.35 and Theorem 2.39 below, the case $q_{0}=\infty$ is understood in the sense that the $L^{q_{0}}(w)$-average is replaced by the essential supremum. Also in Theorem 2.35, if $q_{0}=\infty$, then the condition on $v$ becomes $v \in A_{p / p_{0}}$.

Theorem 2.39. Given $w \in A_{2}$ with doubling order $D$, and $1 \leq p_{0}<q_{0} \leq \infty$, let $T$ : $L^{q_{0}}(w) \rightarrow L^{q_{0}}(w)$ be a sublinear operator, and $\left\{\mathcal{A}_{r}\right\}_{r>0}$ a family of linear operators acting from $L_{c}^{\infty}$ into $L^{q_{0}}(w)$. Suppose that for every ball $B$ with radius $r, f \in L_{c}^{\infty}$ with $\operatorname{supp}(f) \subset B$ and $j \geq 2$,

$$
\left(f_{C_{j}(B)}\left|T\left(I-\mathcal{A}_{r}\right) f\right|^{p_{0}} d w\right)^{1 / p_{0}} \leq g(j)\left(f_{B}|f|^{p_{0}} d w\right)^{1 / p_{0}} .
$$

Suppose further that for every $j \geq 1$,

$$
\left(f_{C_{j}(B)}\left|\mathcal{A}_{r} f\right|^{q_{0}} d w\right)^{1 / q_{0}} \leq g(j)\left(f_{B}|f|^{p_{0}} d w\right)^{1 / p_{0}}
$$

where $\sum g(j) 2^{D j}<\infty$. Then for all $p, p_{0}<p<q_{0}$, there exists a constant $C$ such that for all $f \in L_{c}^{\infty}$,

$$
\|T f\|_{L^{p}(w)} \leq C\|f\|_{L^{p}(w)}
$$

\section{OfF-Diagonal estimates FOR the SEMigroup $e^{-t L_{w}}$}

In this section we consider off-diagonal estimates for the semigroup associated to $L_{w}$. Throughout this and subsequent sections, let $w \in A_{2}$ and $A \in \mathcal{E}_{n}(w, \Lambda, \lambda)$ be fixed. Our goal is to characterize the set of pairs $(p, q), p \leq q$ such that these operators are in $\mathcal{O}\left(L^{p}(w) \rightarrow\right.$ $\left.L^{q}(w)\right)$. By Theorem 2.15 we have that

$$
e^{-t L_{w}} \in \mathcal{F}\left(L^{2}(w) \rightarrow L^{2}(w)\right) \subset \mathcal{O}\left(L^{2}(w) \rightarrow L^{2}(w)\right) .
$$

We will show that in the $(p, q)$-plane this set contains a right triangle: see Figure 1.

Figure 1. $(p, q)$ such that $e^{-t L_{w}} \in \mathcal{O}\left(L^{p}(w) \rightarrow L^{q}(w)\right)$

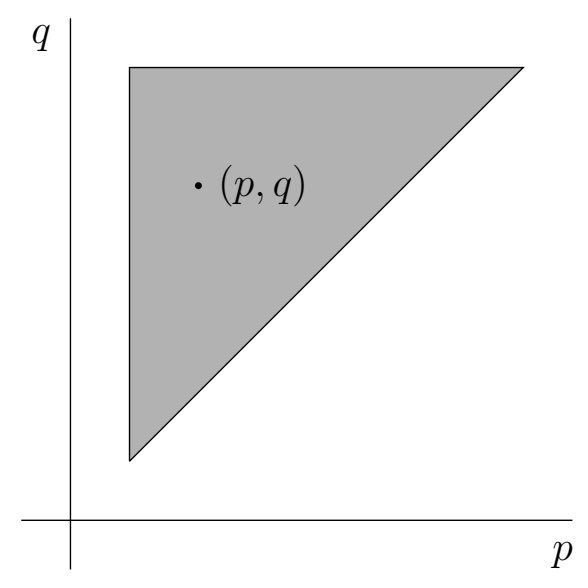

Let $\tilde{\mathcal{J}}\left(L_{w}\right) \subset[1, \infty]$ be the set of all exponents $p$ such that $e^{-t L_{w}}: L^{p}(w) \rightarrow L^{p}(w)$ is uniformly bounded for all $t>0$. By Theorem 2.15 and Lemma $2.34,2 \in \widetilde{\mathcal{J}}\left(L_{w}\right)$, and if it 
contains more than one point, then by interpolation $\widetilde{\mathcal{J}}\left(L_{w}\right)$ is an interval. The set of pairs $(p, q)$ such that $e^{-t L_{w}} \in \mathcal{O}\left(L^{p}(w) \rightarrow L^{p}(w)\right)$ is completely characterized by the next result.

Proposition 3.1. There exists an interval $\mathcal{J}\left(L_{w}\right) \subset[1, \infty]$ such that $p, q \in \mathcal{J}\left(L_{w}\right)$ if and only if $e^{-t L_{w}} \in \mathcal{O}\left(L^{p}(w) \rightarrow L^{q}(w)\right)$. Furthermore, $\mathcal{J}\left(L_{w}\right)$ has the following properties:

(1) $\mathcal{J}\left(L_{w}\right) \subset \widetilde{\mathcal{J}}\left(L_{w}\right)$;

(2) $\operatorname{Int} \mathcal{J}\left(L_{w}\right)=\operatorname{Int} \tilde{\mathcal{J}}\left(L_{w}\right)$;

(3) if $p_{-}\left(L_{w}\right)$ and $p_{+}\left(L_{w}\right)$ are respectively the left and right endpoints of $\mathcal{J}\left(L_{w}\right)$, then $p_{-}\left(L_{w}\right) \leq\left(2_{w}^{*}\right)^{\prime}$ and $p_{+}\left(L_{w}\right) \geq 2_{w}^{*}$, where $2_{w}^{*}$ is as in Theorem 2.1. In particular, $2 \in \operatorname{Int}\left(\mathcal{J}\left(L_{w}\right)\right)$.

Remark 3.2. The smaller the value of $r_{w}$, the better our bounds on the size of the set $\mathcal{J}\left(L_{w}\right)$. In the limiting case when $w \in A_{1}$, we have that $p_{-}\left(L_{w}\right) \leq \frac{2 n}{n+2}$ and $p_{+}\left(L_{w}\right) \geq \frac{2 n}{n-2}$. These values should be compared to the estimates in [2, Corollary 4.6] for the non-degenerate case that corresponds to the case $w=1$.

We get two corollaries to Proposition 3.1. The first gives us weighted off-diagonal estimates.

Corollary 3.3. Let $p_{-}\left(L_{w}\right)<p \leq q<p_{+}\left(L_{w}\right)$. If $v \in A_{p^{\prime} p_{-}\left(L_{w}\right)}(w) \cap R H_{\left(p_{+}\left(L_{w}\right) / q\right)^{\prime}}(w)$, then $e^{-t L_{w}} \in \mathcal{O}\left(L^{p}(v d w) \rightarrow L^{q}(v d w)\right)$.

Proof. By Proposition 3.1, if $p_{-}\left(L_{w}\right)<p \leq q<p_{+}\left(L_{w}\right)$, then $e^{-t L_{w}} \in \mathcal{O}\left(L^{p}(w) \rightarrow L^{q}(w)\right)$. Therefore, by [7, Proposition 2.6], if $v \in A_{p / p_{-}\left(L_{w}\right)}(w) \cap R H_{\left(p_{+}\left(L_{w}\right) / q\right)^{\prime}}(w)$, then we have that $e^{-t L_{w}} \in \mathcal{O}\left(L^{p}(v d w) \rightarrow L^{q}(v d w)\right)$.

As our second corollary we get off-diagonal estimates for the holomorphic extension of the semigroup.

Corollary 3.4. For any $\nu, 0<\nu<\frac{\pi}{2}-\vartheta$, and for any $p \leq q$ such that $e^{-t L_{w}} \in \mathcal{O}\left(L^{p}(w) \rightarrow\right.$ $\left.L^{q}(w)\right)$, then for all $m \in \mathbb{N} \cup\{0\},\left(z L_{w}\right)^{m} e^{-z L_{w}} \in \mathcal{O}\left(L^{p}(w) \rightarrow L^{q}(w), \Sigma_{\nu}\right)$.

Proof. This follows from [7, Theorem 4.3] and the fact that, by Theorem 2.15, for these values of $z, e^{-z L_{w}} \in \mathcal{F}\left(L^{2}(w) \rightarrow L^{2}(w)\right)$.

Proof of Proposition 3.1. Fix $2<q<2_{w}^{*}$ (If $w \in A_{1}$ we let $q=2_{w}^{*}=2^{*}$.) We will show that $e^{-t L_{w}} \in \mathcal{O}\left(L^{2}(w) \rightarrow L^{q}(w)\right)$. Given this, then we also have that $e^{-t L_{w}} \in \mathcal{O}\left(L^{q^{\prime}}(w) \rightarrow\right.$ $\left.L^{2}(w)\right)$. For if $L_{w}^{*}$ is the adjoint of $L_{w}$ (with respect to $L^{2}(w)$ ), then $L_{w}^{*}=-w^{-1} \operatorname{div}\left(A^{*} \nabla f\right)$ and the same estimates hold for $L_{w}^{*}$. Hence, $e^{-t L_{w}^{*}} \in \mathcal{O}\left(L^{2}(w) \rightarrow L^{q}(w)\right)$, and so by Lemma 2.29, $e^{-t L_{w}} \in \mathcal{O}\left(L^{q^{\prime}}(w) \rightarrow L^{2}(w)\right)$. Since $e^{-t L_{w}}$ is a semigroup, by Lemma 2.30 we have that $e^{-t L_{w}} \in \mathcal{O}\left(L^{q^{\prime}}(w) \rightarrow L^{q}(w)\right)$. Therefore, by [7, Proposition 4.1], we have that there exists an interval $\mathcal{J}\left(L_{w}\right)$ and Properties (1) and (2) hold. Moreover, we have that $\left[q^{\prime}, q\right] \subset \mathcal{J}\left(L_{w}\right)$, so if we let $q \rightarrow 2_{w}^{*}$, then we immediately get Property (3).

It therefore remains to prove that $e^{-t L_{w}} \in \mathcal{O}\left(L^{2}(w) \rightarrow L^{q}(w)\right)$. We first show (2.24). Fix $B$ and for brevity write $r=r(B)$ and $C_{j}=C_{j}(B)$. By our choice of $q$ the Poincaré inequality (2.3) holds. Moreover, as we noted above $e^{-t L_{w}}, \sqrt{t} \nabla e^{-t L_{w}} \in \mathcal{O}\left(L^{2}(w) \rightarrow L^{2}(w)\right)$; we 
may assume that the same exponents $\theta_{1}, \theta_{2}$ hold for both operators. We thus get that

$$
\begin{aligned}
\left(f_{B} \mid\right. & \left.\left.e^{-t L_{w}}\left(\chi_{B} f\right)\right|^{q} d w\right)^{\frac{1}{q}} \\
& \leq\left|\left(e^{-t L_{w}}\left(\chi_{B} f\right)\right)_{B, w}\right|+\left(f_{B}\left|e^{-t L_{w}}\left(\chi_{B} f\right)(x)-\left(e^{-t L_{w}}\left(\chi_{B} f\right)\right)_{B, w}\right|^{q} d w(x)\right)^{\frac{1}{q}} \\
& \lesssim\left(f_{B}\left|e^{-t L_{w}}\left(\chi_{B} f\right)\right|^{2} d w\right)^{\frac{1}{2}}+r\left(f_{B}\left|\nabla e^{-t L_{w}}\left(\chi_{B} f\right)\right|^{2} d w\right)^{\frac{1}{2}} \\
& \lesssim\left(1+\frac{r}{\sqrt{t}}\right) \Upsilon\left(\frac{r}{\sqrt{t}}\right)^{\theta_{2}}\left(f_{B}|f|^{2} d w\right)^{\frac{1}{2}} \\
& \lesssim \Upsilon\left(\frac{r}{\sqrt{t}}\right)^{1+\theta_{2}}\left(f_{B}|f|^{2} d w\right)^{\frac{1}{2}} .
\end{aligned}
$$

The proof that (2.25) holds is gotten by nearly the same argument:

$$
\begin{aligned}
\left(f_{B}\left|e^{-t L_{w}}\left(\chi_{C_{j}} f\right)\right|^{q} d w\right)^{\frac{1}{q}} & \\
& \leq\left|\left(e^{-t L_{w}}\left(\chi_{C_{j}} f\right)\right)_{B, w}\right|+\left(f_{B}\left|e^{-t L_{w}}\left(\chi_{C_{j}} f\right)(x)-\left(e^{-t L_{w}}\left(\chi_{C_{j}} f\right)\right)_{B, w}\right|^{q} d w(x)\right)^{\frac{1}{q}} \\
& \lesssim\left(f_{B}\left|e^{-t L_{w}}\left(\chi_{C_{j}} f\right)\right|^{2} d w\right)^{\frac{1}{2}}+r\left(f_{B}\left|\nabla e^{-t L_{w}}\left(\chi_{C_{j}} f\right)\right|^{2} d w\right)^{\frac{1}{2}} \\
& \lesssim 2^{j \theta_{1}}\left(1+\frac{r}{\sqrt{t}}\right) \Upsilon\left(\frac{2^{j} r}{\sqrt{t}}\right)^{\theta_{2}} e^{-\frac{c 4^{j} r^{2}}{t}}\left(f_{C_{j}}|f|^{2} d w\right)^{\frac{1}{2}} \\
& \lesssim 2^{j \theta_{1}} \Upsilon\left(\frac{2^{j} r}{\sqrt{t}}\right)^{1+\theta_{2}} e^{-\frac{c 4^{j} r^{2}}{t}}\left(f_{C_{j}}|f|^{2} d w\right)^{\frac{1}{2}}
\end{aligned}
$$

Finally, to prove that (2.26) holds we use a covering argument. Fix $j \geq 2$; then we can cover the annulus $C_{j}$ by a collection of balls $\left\{B_{k}\right\}_{k=1}^{N}, r\left(B_{k}\right)=2^{j-2} r$, with centers $x_{B_{k}} \in C_{j}$. The number of balls required, $N$, depends only on the dimension. For any such ball, since $d w$ is a doubling measure we have that

$$
\begin{aligned}
& \left(f_{B_{k}}\left|e^{-t L_{w}}\left(\chi_{B} f\right)\right|^{q} d w\right)^{\frac{1}{q}} \\
& \quad \leq\left|\left(e^{-t L_{w}}\left(\chi_{B} f\right)\right)_{B_{k}, w}\right|+\left(f_{B_{k}}\left|e^{-t L_{w}}\left(\chi_{B} f\right)(x)-\left(e^{-t L_{w}}\left(\chi_{B} f\right)\right)_{B_{k}, w}\right|^{q} d w(x)\right)^{\frac{1}{q}} \\
& \quad \lesssim\left(f_{B_{k}}\left|e^{-t L_{w}}\left(\chi_{B} f\right)\right|^{2} d w\right)^{\frac{1}{2}}+r\left(B_{k}\right)\left(f_{B_{k}}\left|\nabla e^{-t L_{w}}\left(\chi_{B} f\right)\right|^{2} d w\right)^{\frac{1}{2}} \\
& \quad \lesssim\left(f_{2^{j+2} B \backslash 2^{j-1} B}\left|e^{-t L_{w}}\left(\chi_{B} f\right)\right|^{2} d w\right)^{\frac{1}{2}}
\end{aligned}
$$




$$
+2^{j} r\left(f_{2^{j+2} B \backslash 2^{j-1} B}\left|\nabla e^{-t L_{w}}\left(\chi_{B} f\right)\right|^{2} d w\right)^{\frac{1}{2}} .
$$

If $j \geq 3$, then $2^{j+2} B \backslash 2^{j-1} B=C_{j+1} \cup C_{j} \cup C_{j-1}$; then to estimate the last two terms we use the fact that $e^{-t L_{w}}, \sqrt{t} \nabla e^{-t L_{w}} \in \mathcal{O}\left(L^{2}(w) \rightarrow L^{2}(w)\right)$ and apply (2.26) with $p=q=2$ in each annulus $C_{i}, j-1 \leq i \leq j+1$. (These annuli have comparable measure since $d w$ is a doubling measure so we can divide the average up into three averages). If $j=2$, then $2^{4} B \backslash 2 B=C_{3} \cup C_{2} \cup(4 B \backslash 2 B)$. On $C_{3}$ and $C_{2}$ we argue as before using (2.26). On $4 B \backslash B$ we apply $[7$, Lemma 6.1$]$. (We note that in the notation there $\widehat{C}_{1}(B)=4 B \backslash 2 B$.)

If we combine all of these estimates, we get that for every $j \geq 2$,

$$
\begin{aligned}
\left(f_{B_{k}}\left|e^{-t L_{w}}\left(\chi_{B} f\right)\right|^{q} d w\right)^{\frac{1}{q}} \lesssim 2^{j \theta_{1}}\left(1+\frac{2^{j} r}{\sqrt{t}}\right) \Upsilon\left(\frac{2^{j} r}{\sqrt{t}}\right)^{\theta_{2}}\left(f_{B}|f|^{2} d w\right)^{\frac{1}{2}} \\
\lesssim 2^{j \theta_{1}} \Upsilon\left(\frac{2^{j} r}{\sqrt{t}}\right)^{1+\theta_{2}} e^{-\frac{c 4^{j} r^{2}}{t}}\left(f_{B}|f|^{2} d w\right)^{\frac{1}{2}} .
\end{aligned}
$$

Since $C_{j} \subset \bigcup_{k} B_{k}$, we can sum in $k$ to get

$$
\begin{aligned}
\left(f_{C_{j}(B)}\left|e^{-t L_{w}}\left(\chi_{B} f\right)\right|^{q} d w\right)^{\frac{1}{q}} & \lesssim \sum_{k=1}^{N}\left(f_{B_{k}}\left|e^{-t L_{w}}\left(\chi_{B} f\right)\right|^{q} d w\right)^{\frac{1}{q}} \\
& \lesssim 2^{j \theta_{1}} \Upsilon\left(\frac{2^{j} r}{\sqrt{t}}\right)^{1+\theta_{2}} e^{-\frac{c 4^{j} r^{2}}{t}}\left(f_{B}|f|^{2} d w\right)^{\frac{1}{2}} .
\end{aligned}
$$

This completes the proof that $e^{-t L_{w}} \in \mathcal{O}\left(L^{2}(w) \rightarrow L^{q}(w)\right)$.

\section{The functional calculus}

In this section we show that the operator $L_{w}$ has an $L^{p}(w)$ holomorphic functional calculus. As we discussed in Section 2 above, we know already that if $\varphi$ is a bounded holomorphic function on $\Sigma_{\mu}, \mu \in(\vartheta, \pi)$, then $\varphi\left(L_{w}\right)$ is a bounded operator on $L^{2}(w)$. Recall that for any $\mu \in(\vartheta, \pi)$, we say that $\varphi \in \mathcal{H}_{0}^{\infty}\left(\Sigma_{\mu}\right)$ if for some $c, s>0$

$$
|\varphi(z)| \leq c|z|^{s}(1+|z|)^{-2 s}, \quad z \in \Sigma_{\mu} .
$$

We say that $L_{w}$ has a bounded holomorphic functional calculus on $L^{p}(w)$ if for any such $\varphi$,

$$
\left\|\varphi\left(L_{w}\right) f\right\|_{L^{p}(w)} \leq C\|\varphi\|_{\infty}\|f\|_{L^{p}(w)}, \quad f \in L^{p}(w) \cap L^{2}(w),
$$

where $C$ depends only on $p, w, \vartheta$ and $\mu$ (but not on the decay of $\varphi$ ). By a standard density argument, (4.2) implies that $\varphi\left(L_{w}\right)$ extends to a bounded operator on all of $L^{p}(w)$. Furthermore, we then have that this inequality holds if $\varphi$ is any bounded holomorphic function. For the details of this extension, see [23, 27].

Proposition 4.3. Let $p_{-}\left(L_{w}\right)<p<p_{+}\left(L_{w}\right)$ and $\mu \in(\vartheta, \pi)$. Then for any $\varphi \in \mathcal{H}_{0}^{\infty}\left(\Sigma_{\mu}\right)$,

$$
\left\|\varphi\left(L_{w}\right) f\right\|_{L^{p}(w)} \leq C\|\varphi\|_{\infty}\|f\|_{L^{p}(w)}
$$

with $C$ independent of $\varphi$ and $f$. Hence, $L_{w}$ has a bounded holomorphic functional calculus on $L^{p}(w)$. Moreover, if $v \in A_{p / p_{-}\left(L_{w}\right)}(w) \cap R H_{\left(p_{+}\left(L_{w}\right) / p\right)^{\prime}}(w)$ then $L_{w}$ also has a bounded holomorphic functional calculus on $L^{p}(v d w)$ :

$$
\left\|\varphi\left(L_{w}\right) f\right\|_{L^{p}(v d w)} \leq C\|\varphi\|_{\infty}\|f\|_{L^{p}(v d w)},
$$


with $C$ independent of $\varphi$ and $f$.

Proof. For brevity, let $p_{-}=p_{-}\left(L_{w}\right)$ and $p_{+}=p_{+}\left(L_{w}\right)$. By density it will suffice to assume that $f \in L_{c}^{\infty}$. Fix $\varphi \in \mathcal{H}_{0}^{\infty}\left(\Sigma_{\mu}\right)$; by linearity we may assume that $\|\varphi\|_{\infty}=1$.

We divide the proof into two steps. We first obtain (4.4) for $p_{-}<p<2$ by applying Theorem 2.39 and following the ideas in [2]. To do so, we will pick $q_{0}=2$ and $p_{0}>p_{-}$ arbitrarily close to $p_{-}$. In the second step, using some ideas from [5], we will use Theorem 2.35 to get (4.5); in particular this yields (4.4) for every $2<p<p_{+}$by taking $v \equiv 1$. To apply Theorem 2.35 we will choose $p_{0}>p_{-}$arbitrarily close to $p_{-}$and $q_{0}<p_{+}$arbitrarily close to $p_{+}$. We will also use the fact that $\varphi\left(L_{w}\right)$ is bounded on $L^{p_{0}}(w)$; this follows from the first step choosing $p_{-}<p_{0}<2$.

To apply Theorem 2.39, fix $p_{-}<p_{0}<p<2$ and let $q_{0}=2, T=\varphi\left(L_{w}\right)$, and

$$
\mathcal{A}_{r} f(x)=\left(I-\left(I-e^{-r^{2} L_{w}}\right)^{m}\right) f(x),
$$

where $m$ is a positive integer that will be chosen below. We first show that inequality (2.41) holds. By Proposition 3.1 we have that $e^{-t L_{w}} \in \mathcal{O}\left(L^{p_{0}}(w) \rightarrow L^{2}(w)\right)$. Using

$$
\mathcal{A}_{r}=\sum_{k=1}^{m}\left(\begin{array}{c}
m \\
k
\end{array}\right)(-1)^{k+1} e^{-k r^{2} L_{w}}
$$

and that for each fixed $m$ and $1 \leq k \leq m$

$$
\Upsilon\left(\frac{r}{\sqrt{k} t}\right) \leq \sqrt{m} \Upsilon\left(\frac{r}{t}\right) \text { and } \exp \left(-\frac{c}{k} \frac{4^{j} r^{2}}{t^{2}}\right) \leq \exp \left(-\frac{c}{m} \frac{4^{j} r^{2}}{t^{2}}\right),
$$

Proposition 3.1 implies

$$
\mathcal{A}_{r} \in \mathcal{O}\left(L^{p}(w) \rightarrow L^{q}(w)\right), \quad \text { for all } p_{-}\left(L_{w}\right)<p \leq q<p_{+}\left(L_{w}\right) .
$$

In particular, we have that $\mathcal{A}_{r} \in \mathcal{O}\left(L^{p_{0}}(w) \rightarrow L^{2}(w)\right)$. Thus, given any ball $B$ with radius $r$, if $\operatorname{supp}(f) \subset B$, then for all $j \geq 1$,

$$
\left(f_{C_{j}(B)}\left|\mathcal{A}_{r} f\right|^{2} d w\right)^{1 / 2} \lesssim 2^{j \theta_{1}} \Upsilon\left(2^{j}\right)^{\theta_{2}} e^{-c 4^{j}}\left(f_{B}|f|^{p_{0}} d w\right)^{1 / p_{0}}
$$

This establishes (2.41) with $g(j)=C 2^{j\left(\theta_{1}+\theta_{2}\right)} e^{-c 4^{j}}$, for in this case we have that

$$
\sum_{j \geq 1} 2^{j\left(\theta_{1}+\theta_{2}+D\right)} e^{-c 4^{j}}<\infty
$$

where $D$ is the doubling constant of $w$.

We next prove that $(2.40)$ holds. Since $\varphi(z)\left(1-e^{-r^{2} z}\right)^{m} \in \mathcal{H}_{0}^{\infty}\left(\Sigma_{\{\min \{\mu, \pi / 2\}\}}\right)$, by the functional calculus representation (2.10) we have that

$$
\varphi\left(L_{w}\right)\left(I-\mathcal{A}_{r}\right) f=\int_{\Gamma} e^{-z L_{w}} f \eta(z) d z,
$$

where $\Gamma=\partial \sum_{\frac{\pi}{2}-\theta}$, with $0<\vartheta<\theta<\nu<\min \{\mu, \pi / 2\}$, and we choose $\theta$ so that the hypotheses of Corollary 3.4 are satisfied for $z \in \Gamma$. Moreover, we have the estimate

$$
|\eta(z)| \lesssim \frac{r^{2 m}}{|z|^{m+1}}
$$

see [2, Section 5.1] for details. 
We can now argue as follows: given a ball $B$ with radius $r$, for each $j \geq 2$, by Minkowski's inequality and Corollary 3.4 (since $p_{0} \in \operatorname{Int} \mathcal{J}\left(L_{w}\right)$ ),

$$
\begin{aligned}
\left(f_{C_{j}(B)} \mid \varphi\right. & \left.\left.\left(L_{w}\right)\left(I-\mathcal{A}_{r(B)}\right) f\right|^{p_{0}} d w\right)^{1 / p_{0}} \\
& =\left(f_{C_{j}(B)}\left|\int_{\Gamma} e^{-z L_{w}} f \eta(z) d z\right|^{p_{0}} d w\right)^{1 / p_{0}} \\
& \lesssim \int_{\Gamma}\left(f_{C_{j}(B)}\left|e^{-z L_{w}} f\right|^{p_{0}} d w\right)^{1 / p_{0}} \frac{r^{2 m}}{|z|^{m+1}}|d z| \\
& \lesssim\left(f_{B}|f|^{p_{0}} d w\right)^{1 / p_{0}} \int_{\Gamma} \frac{r^{2 m}}{|z|^{m+1}} 2^{j \theta_{1}} \Upsilon\left(\frac{2^{j} r}{\sqrt{|z|}}\right)^{\theta_{2}} e^{-c \frac{r^{2}}{|z|} 4^{j}}|d z| \\
& =\left(f_{B}|f|^{p_{0}} d w\right)^{1 / p_{0}} 2^{j\left(\theta_{1}-2 m\right)} \int_{0}^{\infty} \sigma^{2 m} \Upsilon(\sigma)^{\theta_{2}} e^{-c \sigma^{2}} \frac{d \sigma}{\sigma} \\
& \lesssim 2^{j\left(\theta_{1}-2 m\right)}\left(f_{B}|f|^{p_{0}} d w\right)^{1 / p_{0}} ;
\end{aligned}
$$

the final inequality holds (i.e., the integral in $\sigma$ converges) provided $2 m>\theta_{2}$. Moreover, if we choose $2 m>\theta_{1}+D$, we have that $(2.40)$ holds with $g(j)=C 2^{(j-1)\left(\theta_{1}-2 m\right)}$ and

$$
\sum_{j \geq 2} g(j) 2^{j D} \lesssim \sum_{j \geq 2} 2^{j\left(\theta_{1}+D-2 m\right)}<\infty .
$$

We have shown that inequalities (2.40) and (2.41) hold, and so by Theorem 2.39 inequality (4.4) holds for all $p$ such that $p_{-}<p \leq 2$.

We will now apply Theorem 2.35 to show that (4.5) holds for $p_{-}<p<p_{+}$. (Inequality (4.4) then follows for $2<p<p_{+}$if we take $v \equiv 1$.) Fix $p, p_{-}<p<p_{+}$and $v \in A_{p / p_{-}}(w) \cap R H_{\left(p_{+} / p\right)^{\prime}}(w)$. By the openness properties of the $A_{q}$ and $R H_{s}$ classes there exist $p_{0}, q_{0}$ such that

$$
p_{-}<p_{0}<\min \{p, 2\} \leq p<q_{0}<p_{+}, \quad v \in A_{p / p_{0}}(w) \cap R H_{\left(q_{0} / p\right)^{\prime}}(w) .
$$

Let $T=\varphi\left(L_{w}\right), \mathcal{A}_{r}=I-\left(I-e^{-r^{2} L_{w}}\right)^{m}, S=I$, and fix the above values of $p_{0}$ and $q_{0}$. By the previous argument we have that $\varphi\left(L_{w}\right)$ is bounded on $L^{p_{0}}(w)$.

We first show that (2.36) holds. Fix a ball $B$ and decompose $f$ as

$$
f=\sum_{j \geq 1} f \chi_{C_{j}(B)}:=\sum_{j \geq 1} f_{j}
$$

Then, by the same functional calculus argument as given above, we have that for each $j$,

$$
\begin{aligned}
\left(f_{B} \mid\right. & \left.\left.\varphi\left(L_{w}\right)\left(I-\mathcal{A}_{r}\right) f_{j}\right|^{p_{0}} d w\right)^{\frac{1}{p_{0}}} \\
= & \left(f_{B}\left|\int_{\Gamma} e^{-z L_{w}} f_{j} \eta(z) d z\right|^{p_{0}} d w\right)^{\frac{1}{p_{0}}} \\
& \lesssim \int_{\Gamma}\left(f_{B}\left|e^{-z L_{w}} f_{j}\right|^{p_{0}} d w\right)^{\frac{1}{p_{0}}} \frac{r^{2 m}}{|z|^{m+1}}|d z|
\end{aligned}
$$




$$
\begin{aligned}
& \lesssim\left(f_{C_{j}(B)}|f|^{p_{0}} d w\right)^{\frac{1}{p_{0}}} 2^{j\left(\theta_{1}-2 m\right)} \int_{\Gamma}\left(\frac{2^{j} r}{\sqrt{|z|}}\right)^{2 m} \Upsilon\left(\frac{2^{j} r}{\sqrt{|z|}}\right)^{\theta_{2}} e^{-\frac{c 4^{j} r^{2}}{|z|}} \frac{|d z|}{|z|} \\
& \lesssim 2^{j\left(\theta_{1}-2 m\right)}\left(f_{C_{j}(B)}|f|^{p_{0}} d w\right)^{\frac{1}{p_{0}}} ;
\end{aligned}
$$

the last inequality holds provided $2 m>\theta_{2}$. Hence, since $2^{j+1} B \supset C_{j}$, by Minkowski's inequality we have (since the sum $\sum f_{j}$ is finite for $f \in L_{c}^{\infty}$ )

$$
\begin{aligned}
\left(f_{B}\left|\varphi\left(L_{w}\right)\left(I-\mathcal{A}_{r}\right) f\right|^{p_{0}} d w\right)^{\frac{1}{p_{0}}} & \leq \sum_{j \geq 1}\left(f_{B}\left|\varphi\left(L_{w}\right)\left(I-\mathcal{A}_{r}\right) f_{j}\right|^{p_{0}} d w\right)^{\frac{1}{p_{0}}} \\
& \lesssim \sum_{j \geq 1} 2^{j\left(\theta_{1}-2 m\right)}\left(f_{2^{j+1} B}|f|^{p_{0}} d w\right)^{\frac{1}{p_{0}}}
\end{aligned}
$$

This establishes $(2.36)$ with $g(j)=C 2^{j\left(\theta_{1}-2 m\right)}$. If we take $2 m>\max \left\{\theta_{1}, \theta_{2}\right\}$, then $\sum g(j)<$ $\infty$.

We now show that (2.37) holds. Fix a ball $B$ and $j \geq 1$. Since $\mathcal{A}_{r} \in \mathcal{O}\left(L^{p_{0}}(w) \rightarrow L^{q_{0}}(w)\right)$ (see (4.8)),

$$
\left(f_{B}\left|\mathcal{A}_{r}\left(\chi_{C_{j}(B)} \varphi\left(L_{w}\right) f\right)\right|^{q_{0}} d w\right)^{\frac{1}{q_{0}}} \lesssim 2^{j \theta_{1}} \Upsilon\left(2^{j}\right)^{\theta_{2}} e^{-c 4^{j}}\left(f_{C_{j}(B)}\left|\varphi\left(L_{w}\right) f\right|^{p_{0}} d \mu\right)^{\frac{1}{p_{0}}}
$$

Therefore, since $\varphi\left(L_{w}\right)$ and $\mathcal{A}_{r}$ commute, by Minkowski's inequality we obtain

$$
\left(f_{B}\left|\varphi\left(L_{w}\right) \mathcal{A}_{r} f\right|^{q_{0}} d w\right)^{\frac{1}{q_{0}}} \lesssim \sum_{j \geq 1} 2^{j\left(\theta_{1}+\theta_{2}\right)} e^{-c 4^{j}}\left(f_{C_{j}(B)}\left|\varphi\left(L_{w}\right) f\right|^{p_{0}} d \mu\right)^{\frac{1}{p_{0}}} .
$$

This establishes (2.37) with $g(j)=C 2^{j\left(\theta_{1}+\theta_{2}\right)} e^{-c 4^{j}}$; again, $\sum g(j)<\infty$. Therefore, our proof is complete.

\section{Square FunCtion estimates for the Semigroup}

In this section we prove $L^{p}(w)$ norm inequalities for the vertical square function associated to the semigroup $e^{-t L_{w}}$ :

$$
g_{L_{w}} f(x)=\left(\int_{0}^{\infty}\left|\left(t L_{w}\right)^{1 / 2} e^{-t L_{w}} f(x)\right|^{2} \frac{d t}{t}\right)^{1 / 2} .
$$

Proposition 5.1. Let $p_{-}\left(L_{w}\right)<p<p_{+}\left(L_{w}\right)$. Then

$$
\left\|g_{L_{w}} f\right\|_{L^{p}(w)} \approx\|f\|_{L^{p}(w)} .
$$

Conversely if for some $p$ the equivalence (5.2) holds, then $p \in \tilde{\mathcal{J}}\left(L_{w}\right)$-i.e., the interior of the interval on which (5.2) holds is $\left(p_{-}\left(L_{w}\right), p_{+}\left(L_{w}\right)\right)$.

Moreover, if $v \in A_{p / p_{-}\left(L_{w}\right)}(w) \cap R H_{\left(p_{+}\left(L_{w}\right) / p\right)^{\prime}}(w)$, then

$$
\left\|g_{L_{w}} f\right\|_{L^{p}(v d w)} \approx\|f\|_{L^{p}(v d w)} .
$$


We note that the upper bounds in the previous result could be obtained by combining Proposition 4.3 with the operator theory methods developed in [14]. To reach a wider audience we present a self-contained harmonic analysis proof. We will use an auxiliary Hilbert space related to square functions, following the approach in [5]. Let $\mathbb{H}$ denote the Hilbert space $L^{2}\left((0, \infty), \frac{d t}{t}\right)$ with norm

$$
\|h\|=\left(\int_{0}^{\infty}|h(t)|^{2} \frac{d t}{t}\right)^{\frac{1}{2}} .
$$

In particular, we have that

$$
g_{L_{w}} f(x)=\|\varphi(L, \cdot) f(x)\|
$$

where $\varphi(z, t)=(t z)^{1 / 2} e^{-t z}$. Furthermore, we define $L_{\mathbb{H}}^{p}(w)$ to be the space of $\mathbb{H}$-valued functions with the norm

$$
\|h\|_{L_{\mathbb{H}}^{p}(w)}=\left(\int_{\mathbb{R}^{n}}\|h(x, \cdot)\|^{p} d w(x)\right)^{\frac{1}{p}} .
$$

The following lemma lets us extend scalar valued inequalities to $\mathbb{H}$-valued inequalities. For a proof, see [5, Lemma 7.4] and the references given there.

Lemma 5.4. Given a Borel measure $\mu$ on $\mathbb{R}^{n}$, let $\mathcal{D}$ be a subspace of $\mathcal{M}$, the space of measurable functions in $\mathbb{R}^{n}$, and let $S, T$ be linear operators from $\mathcal{D}$ into $\mathcal{M}$. Fix $1 \leq p \leq$ $q<\infty$ and suppose there exists $C_{0}>0$ such that for all $f \in \mathcal{D}$,

$$
\|T f\|_{L^{q}(\mu)} \leq C_{0} \sum_{j \geq 1} \alpha_{j}\|S f\|_{L^{p}\left(F_{j}, \mu\right)},
$$

where the $F_{j}$ are measurable subsets of $\mathbb{R}^{n}$ and $\alpha_{j} \geq 0$. Then there is a $\mathbb{H}$-valued inequality with the same constant: for all $f: \mathbb{R}^{n} \times(0, \infty) \longrightarrow \mathbb{C}$ such that for almost all $t>0$, $f(\cdot, t) \in \mathcal{D}$,

$$
\|T f\|_{L_{\mathbb{H}}^{q}(\mu)} \leq C_{0} \sum_{j \geq 1} \alpha_{j}\|S f\|_{L_{\mathbb{H}}^{p}\left(F_{j}, \mu\right)} .
$$

The extension of a linear operator $T$ on $\mathbb{C}$-valued functions to $\mathbb{H}$-valued functions is defined for $x \in \mathbb{R}^{n}$ and $t>0$ by $(T h)(x, t)=T(h(\cdot, t))(x)$, that is, $t$ can be considered as a parameter and $T$ acts only on the variable in $\mathbb{R}^{n}$.

Proof of Proposition 5.1. We shall first prove the upper bound inequalities. We first claim that the upper bound inequality in (5.2) holds for $p=2$. Indeed, since $\varphi(z)=z^{1 / 2} e^{-z} \in$ $\mathcal{H}_{0}^{\infty}\left(\Sigma_{\mu}\right)$, it follows from $(2.13)$ that we have the bound

$$
\left\|g_{L_{w}} f\right\|_{L^{2}(w)} \lesssim\|f\|_{L^{2}(w)} .
$$

For brevity, let $p_{-}=p_{-}\left(L_{w}\right)$ and $p_{+}=p_{+}\left(L_{w}\right)$. As in previous proofs, we divide our proof into two steps. We will first prove the upper bound in (5.2) for $p_{-}<p<2$ by applying Theorem 2.39. Fix $p_{-}<p<q_{0}=2$, and let $\mathcal{A}_{r}=I-\left(I-e^{-r^{2} L_{w}}\right)^{m}$, where $m$ will be chosen below. Notice that, by $(4.8), \mathcal{A}_{r}$ is bounded on $L^{q_{0}}(w)$ for each $m$. Fix $f \in L_{c}^{\infty}$; the result for general $f \in L^{p}(w)$ then follows by a density argument.

We have that $\left(t L_{w}\right)^{1 / 2} e^{-t L_{w}}\left(I-\mathcal{A}_{r}\right) f=\varphi\left(L_{w}, t\right) f$, where

$$
\varphi(z, t)=(t z)^{1 / 2} e^{-t z}\left(1-e^{-r^{2} z}\right)^{m} .
$$


Moreover, since $\varphi(\cdot, t) \in \mathcal{H}_{0}^{\infty}\left(\Sigma_{\{\min \{\mu, \pi / 2\}\}}\right)$, by the functional calculus representation $(2.10)$ we have that

$$
\left(t L_{w}\right)^{1 / 2} e^{-t L_{w}}\left(I-\mathcal{A}_{r}\right) f=\int_{\Gamma} \eta(z, t) e^{-z L_{w}} f d z
$$

where $\Gamma=\partial \Sigma_{\frac{\pi}{2}-\theta}$, with $0<\vartheta<\theta<\nu<\min \{\mu, \pi / 2\}$, and we choose $\theta$ so that the hypotheses of Corollary 3.4 are satisfied for $z \in \Gamma$. Moreover, we have the estimate (see $[2,5])$

therefore,

$$
|\eta(z, t)| \lesssim \frac{t^{\frac{1}{2}} r^{2 m}}{(|z|+t)^{m+\frac{3}{2}}}, \quad z \in \Gamma
$$

$$
\|\eta(z, \cdot)\|=\left(\int_{0}^{\infty}|\eta(z, t)|^{2} \frac{d t}{t}\right)^{1 / 2} \lesssim \frac{r^{2 m}}{|z|^{m+1}} .
$$

Now let $f \in L_{c}^{\infty}$ with $\operatorname{supp}(f) \subset B$. For $j \geq 2$, we have

$$
\begin{aligned}
\left(f_{C_{j}(B)}\left|g_{L_{w}}\left(I-\mathcal{A}_{r}\right) f\right|^{p} d w\right)^{1 / p} & \left(f_{C_{j}(B)}\left|\left(\int_{0}^{\infty}\left|\int_{\Gamma_{\frac{\pi}{2}-\theta}} \eta(z, t) e^{-z L_{w}} f d z\right|^{2} \frac{d t}{t}\right)^{1 / 2}\right|^{p} d w\right)^{1 / p} \\
& \leq\left(f_{C_{j}(B)}\left|\int_{\Gamma_{\frac{\pi}{2}-\theta}}\right| e^{-z L_{w}} f|\| \eta(z, \cdot)||d| z||^{p} d w\right)^{1 / p} \\
& \lesssim \int_{\Gamma_{\frac{\pi}{2}-\theta}}\left(f_{C_{j}(B)}\left|e^{-z L_{w}} f\right|^{p} d w\right)^{1 / p} \frac{r^{2 m}}{|z|^{m+1}} d|z| \\
& \lesssim 2^{j \theta_{1}}\left(f_{B}|f|^{p} d w\right)^{1 / p} \int_{\Gamma_{\frac{\pi}{2}-\theta}} \Upsilon\left(\frac{2^{j} r}{\sqrt{|z|}}\right)^{\theta_{2}} e^{-\frac{c 4^{j} r^{2}}{|z|}} \frac{r^{2 m}}{|z|^{m}} \frac{d|z|}{|z|} \\
& \lesssim 2^{j \theta_{1}} 4^{-m j}\left(f_{B}|f|^{p} d w\right)^{1 / p} ;
\end{aligned}
$$

in the second inequality we applied (5.5) and the off-diagonal estimates for $e^{-z L_{w}}$ from Corollary 3.4, and the last inequality holds provided $2 m>\theta_{2}$. Thus, if we take $m>\theta_{1}+D$, where $D$ is the doubling order of $w$, the operator $g_{L_{w}}$ satisfies (2.40) in Theorem 2.39 with $g(j)=C 2^{j\left(\theta_{1}-2 m\right)}$. Since we already established $(2.41)$ in $(4.9)$ with $g(j)=C 2^{j\left(\theta_{1}+\theta_{2}\right)} 4^{-m j}$, the hypotheses of Theorem 2.39 are satisfied if $m>\theta_{1}+\theta_{2}+D$. Therefore, for each $p_{-}<p<2$ there exists a constant $C$ such that

$$
\left\|g_{L_{w}} f\right\|_{L^{p}(w)} \leq C\|f\|_{L^{p}(w)} .
$$

In the second part of the proof we will show that if $p_{-}<p<p_{+}$and $v \in A_{p / p_{-}}(w) \cap$ $R H_{\left(p_{+} / p\right)^{\prime}}(w)$, then the upper bound inequality in (5.3) holds. If we take $v \equiv 1$, then we immediately get (5.2). To do so, first note that if we fix $p$ and $v$, then by the openness properties of weights there exist $p_{0}, q_{0}$ such that

$$
p_{-}<p_{0}<\min \{p, 2\} \leq \max \{p, 2\}<q_{0}<p_{+}
$$

and $v \in A_{p_{0} / p_{-}}(w) \cap R H_{\left(q_{0} / p\right)^{\prime}}(w)$. 
We will apply Theorem 2.35 with $T=g_{L_{w}}, S=I$ and $\mathcal{D}=L^{p_{0}}(w)$ (again, note that by (4.8), $\mathcal{A}_{r}$ is bounded on $\left.L^{p_{0}}(w)\right)$. We first prove that inequality (2.36) holds. For each $j \geq 1$, let $f_{j}=f \chi_{C_{j}(B)}$; then we can argue exactly as we did in the proof of (5.6), exchanging the roles of $B$ and $C_{j}(B)$, to get

$$
\left(f_{B}\left|g_{L_{w}}\left(I-\mathcal{A}_{r}\right) f_{j}\right|^{p} d w\right)^{\frac{1}{p}} \lesssim 2^{j \theta_{1}} 4^{-m j}\left(f_{2^{j+1} B}|f|^{p} d w\right)^{\frac{1}{p}} .
$$

Inequality (2.36) follows if we sum over all $j$ and take $g(j)=2^{j \theta_{1}} 4^{-m j}$.

We will now show that inequality (2.37) holds. To do so, we need to prove a vector-valued version of a key inequality. By Proposition 3.1, given a ball $B$ with radius $r$, then for all $j \geq 1, g$ with $\operatorname{supp}(g) \subset C_{j}(B)$, and $1 \leq k \leq m$,

$$
\left(f_{B}\left|e^{-k r^{2} L_{w}} g\right|^{q_{0}} d w\right)^{\frac{1}{q_{0}}} \leq C_{0} 2^{j\left(\theta_{1}+\theta_{2}\right)} e^{-\alpha 4^{j}}\left(f_{C_{j}(B)}|g|^{p_{0}} d w\right)^{\frac{1}{p_{0}}} .
$$

We now apply Lemma 5.4 with $S=I$ and $T: L^{p_{0}}(w) \rightarrow L^{q_{0}}(w)$ given by

$$
T g=\left(C_{0} 2^{j\left(\theta_{1}+\theta_{2}\right)} e^{-\alpha 4^{j}}\right)^{-1} \frac{w\left(2^{j+1} B\right)^{\frac{1}{p_{0}}}}{w(B)^{\frac{1}{q_{0}}}} \chi_{B} e^{-k r^{2} L_{w}}\left(g \chi_{C_{j}(B)}\right) .
$$

This yields the $\mathbb{H}$-valued extension of (5.8): for all $g \in L_{\mathbb{H}}^{p_{0}}(w)$ with $\operatorname{supp}(g(\cdot, t)) \subset C_{j}(B)$, $t>0$, we have that

$$
\left(f_{B}\left\|e^{-k r^{2} L_{w}} g(x, \cdot)\right\|^{q_{0}} d w\right)^{\frac{1}{q_{0}}} \leq C_{0} 2^{j\left(\theta_{1}+\theta_{2}\right)} e^{-\alpha 4^{j}}\left(f_{C_{j}(B)}\|g(x, \cdot)\|^{p_{0}} d w\right)^{\frac{1}{p_{0}}} .
$$

Given an arbitrary $g \in L_{\mathbb{H}}^{p_{0}}(w)$, decompose it as

$$
g(x, t)=\sum_{j \geq 1} g(x, t) \chi_{C_{j}(B)}(x)=\sum_{j \geq 1} g_{j}(x, t) .
$$

Then inequality (5.9) yields

$$
\begin{aligned}
\left(f_{B}\left\|e^{-k r^{2} L_{w}} g(x, \cdot)\right\|^{q_{0}} d w\right)^{\frac{1}{q_{0}}} \leq \sum_{j \geq 1} & \left(f_{B}\left\|e^{-k r^{2} L_{w}} g_{j}(x, \cdot)\right\| \|^{q_{0}} d w\right)^{\frac{1}{q_{0}}} \\
& \lesssim \sum_{j \geq 1} 2^{j\left(\theta_{1}+\theta_{2}\right)} e^{-\alpha 4^{j}}\left(f_{2^{j+1} B}\|g(x, \cdot)\|^{p_{0}} d w\right)^{\frac{1}{p_{0}}} .
\end{aligned}
$$

Define $g(x, t)=\left(t L_{w}\right)^{1 / 2} e^{-t L_{w}} f(x)$. Then $g_{L_{w}} f(x)=\|g(x, \cdot)\| ;$ by our choice of $p_{0}$ and the first step of the proof we have that $g \in L_{\mathbb{H}}^{p_{0}}(w)$. Moreover, since for each $t>0,\left(t L_{w}\right)^{1 / 2} e^{-t L_{w}}$ and $e^{-k r^{2} L_{w}}$ commute,

$$
g_{L_{w}}\left(e^{-k r^{2} L_{w}} f\right)(x)=\left\|e^{-k r^{2} L_{w}} g(x, \cdot)\right\| .
$$

We can now use (4.7) and (5.10) to get that

$$
\left(f_{B}\left|g_{L_{w}} \mathcal{A}_{r} f\right|^{q_{0}} d w\right)^{\frac{1}{q_{0}}} \lesssim \sum_{k=1}^{m}\left(f_{B}\left\|e^{-k r^{2} L_{w}} g(x, \cdot)\right\|^{q_{0}} d w\right)^{\frac{1}{q_{0}}}
$$




$$
\lesssim \sum_{j \geq 1} 2^{j\left(\theta_{1}+\theta_{2}\right)} e^{-\alpha 4^{j}}\left(f_{2^{j+1} B}\left|g_{L_{w}} f\right|^{p} d w\right)^{1 / p_{0}}
$$

This proves $(2.37)$ with $g(j)=C 2^{j\left(\theta_{1}+\theta_{2}\right)} e^{-c 4^{j}}$. Therefore, by Theorem 2.35 we get that

$$
\left\|g_{L_{w}} f\right\|_{L^{p}(v d w)} \lesssim\|f\|_{L^{p}(v d w)}
$$

It remains to show the reverse inequalities. We will prove the lower bound in (5.3); then the lower bound in (5.2) holds if we take $v \equiv 1$. Fix $p_{-}<p<p_{+}$and $v \in A_{p / p_{-}\left(L_{w}\right)}(w) \cap$ $R H_{\left(p_{+}\left(L_{w}\right) / p\right)^{\prime}}(w)$. By the duality properties of weights [6, Lemma 4.4] and since $p_{ \pm}\left(L_{w}\right)^{\prime}=$ $p_{\mp}\left(L_{w}^{*}\right)$, where $L_{w}^{*}$ is the adjoint (on $\left.L^{2}(w)\right)$ of $L_{w}$,

$$
v^{1-p^{\prime}} \in A_{p^{\prime} / p_{-}\left(L^{*}\right)}(w) \cap R H_{\left(p_{+}\left(L^{*}\right) / p^{\prime}\right)^{\prime}}(w) .
$$

We now proceed as in the proof of [5, Theorem 7.3]. Given $F \in L_{\mathbb{H}}^{p}(v d w) \cap L_{\mathbb{H}}^{2}(w)$, and $x \in \mathbb{R}^{n}$ we set

$$
T_{L_{w}} F(x)=\int_{0}^{\infty}\left(t L_{w}\right)^{1 / 2} e^{-t L_{w}} F(x, t) \frac{d t}{t} .
$$

Recall that $\left(t L_{w}\right)^{1 / 2} e^{-t L_{w}} F(x, t)=\left(t L_{w}\right)^{1 / 2} e^{-t L_{w}}(F(\cdot, t))(x)$. Hence, $T_{L_{w}}$ maps $\mathbb{H}$-valued functions to $\mathbb{C}$-valued functions. For $h \in L^{p^{\prime}}\left(v^{1-p^{\prime}} d w\right) \cap L^{2}(w)$ with $\|h\|_{L^{p^{\prime}\left(v^{1-p^{\prime}} d w\right)}}=1$, we have that

$$
\begin{aligned}
\left|\int_{\mathbb{R}^{n}} T_{L_{w}} F \bar{h} d w\right| & =\left|\int_{\mathbb{R}^{n}} \int_{0}^{\infty} F(x, t) \overline{\left(t L_{w}^{*}\right)^{1 / 2} e^{-t L_{w}^{*} h(x)}} \frac{d t}{t} d w(x)\right| \\
& \leq \int_{\mathbb{R}^{n}}\|F(x, \cdot)\| g_{L_{w}^{*}} h(x) d w(x) \\
& \lesssim\|F\|_{L_{\mathbb{H}}^{p}(v d w)}\left\|g_{L_{w}^{*}} h\right\|_{L^{p^{\prime}\left(v^{1-p^{\prime}} d w\right)}} \\
& \lesssim\|F\|_{L_{\mathbb{H}}^{p}(v d w)}
\end{aligned}
$$

where the last estimate uses the fact that $g_{L_{w}^{*}}$ is bounded on $L^{p^{\prime}}\left(v^{1-p^{\prime}} d w\right)$. This follows from the upper bound in (5.3) (with $L_{w}^{*}$ in place of $L_{w}$ ), which we proved above, and (5.11). Taking the supremum over all such functions $h$ and using an standard density argument we have obtained that $T_{L_{w}}$ is bounded from $L_{\mathbb{H}}^{p}(v d w)$ to $L^{p}(v d w)$.

Next, given $f \in L^{p}(v d w) \cap L^{2}(d w)$, if we define $F(x, t)=\left(t L_{w}\right)^{1 / 2} e^{-t L_{w}} f(x)$, then $F \in$ $L_{\mathbb{H}}^{p}(v d w) \cap L_{\mathbb{H}}^{2}(w)$ since $\|F\|_{L_{\mathbb{H}}^{p}(v d w)}=\left\|g_{L_{w}} f\right\|_{L^{p}(v d w)}$ and analogously for $L^{2}(w)$. Also, by the $L^{2}(w)$ functional calculus we have that

$$
f(x)=2 \int_{0}^{\infty}\left(t L_{w}\right)^{1 / 2} e^{-t L_{w}} F(x, t) \frac{d t}{t}=2 T_{L_{w}} F(x) .
$$

Therefore,

$$
\|f\|_{L^{p}(v d w)}=2\left\|T_{L_{w}} F\right\|_{L^{p}(v d w)} \lesssim\|F\|_{L_{\mathbb{H}}^{p}(v d w)}=\left\|g_{L_{w}} f\right\|_{L^{p}(v d w)}
$$

and this completes the proof of (5.3).

To finish the proof of Proposition 5.1 we need to show that the equivalence of norms in (5.2) implies that the semigroup is uniformly bounded. However, this follows immediately from the definition of $g_{L_{w}}$ and the semigroup property: for any $s>0$,

$$
g_{L_{w}}\left(e^{-s L_{w}} f\right)(x)=\left(\int_{0}^{\infty}\left|L_{w}^{1 / 2} e^{-(s+t) L_{w}} f(x)\right|^{2} d t\right)^{1 / 2} \leq g_{L_{w}} f(x) .
$$


This completes the proof.

We conclude this section by proving a version of Proposition 5.1 for the "adjoint" of a discrete square function. We will need this estimate in the proof of Proposition 6.1 below.

Proposition 5.14. Define the holomorphic function $\psi$ on the sector $\Sigma_{\pi / 2}$ by

$$
\psi(z)=\frac{1}{\sqrt{\pi}} \int_{1}^{\infty} z e^{-t z} \frac{d t}{\sqrt{t}}
$$

If $p_{-}\left(L_{w}\right)<p<p_{+}\left(L_{w}\right)$, then for any sequence of functions $\left\{\beta_{k}\right\}_{k \in \mathbb{Z}}$,

$$
\left\|\sum_{k \in \mathbb{Z}} \psi\left(4^{k} L_{w}\right) \beta_{k}\right\|_{L^{p}(w)} \lesssim\left\|\left(\sum_{k \in \mathbb{Z}}\left|\beta_{k}\right|^{2}\right)^{\frac{1}{2}}\right\|_{L^{p}(w)} .
$$

Proof. By duality and since $p_{ \pm}\left(L_{w}\right)^{\prime}=p_{\mp}\left(L_{w}^{*}\right)$, it will suffice to show that for every $p_{-}\left(L_{w}^{*}\right)<$ $p<p_{+}\left(L_{w}^{*}\right)$

$$
\left\|\left(\sum_{k \in \mathbb{Z}}\left|\bar{\psi}\left(4^{k} L_{w}^{*}\right) h\right|^{2}\right)^{\frac{1}{2}}\right\|_{L^{p}(w)} \lesssim\|h\|_{L^{p}(w)},
$$

The function $\psi$ satisfies $|\psi(z)| \leq C|z|^{1 / 2} e^{-c|z|}$ uniformly on subsectors $\Sigma_{\mu}, 0 \leq \mu<\frac{\pi}{2}$. Thus the operator on the lefthand side of (5.17) is a discrete analog of the square function $g_{L_{w}^{*}}$ changing continuous times $t$ to discrete times $4^{k}$ and $z^{1 / 2} e^{-z}$ to $\bar{\psi}(z)$. Since $\bar{\psi}(z)$ has the same quantitative properties as $z^{1 / 2} e^{-z}$ (decay at 0 and at infinity), we can repeat the previous argument and obtain the desired estimates as in the proof of Proposition 5.1.

Remark 5.18. In Proposition 5.14 we can also get $L^{p}(v d w)$ estimates, but in the proof of Proposition 6.1 below we will only need the unweighted estimates. Further details and the precise statements are left to the interested reader.

\section{REVERSE INEQUALities}

In this section we will prove $L^{p}(w)$ estimates of the form $\left\|L_{w}^{1 / 2} f\right\|_{L^{p}(w)} \leq C\|\nabla f\|_{L^{p}(w)}$, which generalize the $L^{2}(w)$ Kato estimates in Theorem 2.18. These are referred to as reverse inequalities since if we replace $f$ by $L_{w}^{-1 / 2} f$, then formally we get a reverse-type inequality for the Riesz transform: $\|f\|_{L^{p}(w)} \leq C\left\|\nabla L_{w}^{-1 / 2} f\right\|_{L^{p}(w)}$.

Since these estimates involve the gradient, in proving them we will rely (implicitly and explicitly) on the weighted Poincaré inequality (2.3). This will require an additional assumption on $p$ when $p<2$. To state it simply, define

$$
\left(p_{-}\left(L_{w}\right)\right)_{w, *}=\frac{n r_{w} p_{-}\left(L_{w}\right)}{n r_{w}+p_{-}\left(L_{w}\right)}<p_{-}\left(L_{w}\right) .
$$

Proposition 6.1. Let $\max \left\{r_{w},\left(p_{-}\left(L_{w}\right)\right)_{w, *}\right\}<p<p_{+}\left(L_{w}\right)$. Then for all $f \in \mathcal{S}$,

$$
\left\|L_{w}^{1 / 2} f\right\|_{L^{p}(w)} \leq C\|\nabla f\|_{L^{p}(w)} .
$$

with $C$ independent of $f$. Furthermore, if $\max \left\{r_{w}, p_{-}\left(L_{w}\right)\right\}<p<p_{+}\left(L_{w}\right)$ and $v \in$ $A_{p / \max \left\{r_{w}, p_{-}\left(L_{w}\right)\right\}}(w) \cap R H_{\left(p_{+}\left(L_{w}\right) / p\right)^{\prime}}(w)$, then for all $f \in \mathcal{S}$,

$$
\left\|L_{w}^{1 / 2} f\right\|_{L^{p}(v d w)} \leq C\|\nabla f\|_{L^{p}(v d w)} .
$$


Remark 6.4. The quantity $\max \left\{r_{w},\left(p_{-}\left(L_{w}\right)\right)_{w, *}\right\}$ can be equal to either term. For instance, it equals $r_{w}$ if $p_{-}\left(L_{w}\right) \leq n^{\prime} r_{w}$. From Proposition 3.1 we know that $p_{-}\left(L_{w}\right)<\left(2_{w}^{*}\right)^{\prime}=\frac{2 n r_{w}}{n r_{w}+2}$, but this only implies the previous inequality for some values of $n$ and $r_{w}$.

Proof. As before, let $p_{-}=p_{-}\left(L_{w}\right)$ and $p_{+}=p_{+}\left(L_{w}\right)$. Fix $p, \max \left\{r_{w},\left(p_{-}\right)_{w, *}\right\}<p<2$, and $f \in \mathcal{S}$. We will first show that

$$
\left\|L_{w}^{1 / 2} f\right\|_{L^{p, \infty}(w)} \lesssim\|\nabla f\|_{L^{p}(w)}
$$

First note that since $p>r_{w}, w \in A_{p}$. Therefore, given $\alpha>0$ we can form the CalderónZygmund decomposition given in [5, Lemma 6.6]: there exist a collection of balls $\left\{B_{i}\right\}_{i}$, smooth functions $\left\{b_{i}\right\}_{i}$ and a function $g \in L_{\mathrm{loc}}^{1}(w)$ such that

$$
f=g+\sum_{i} b_{i}
$$

and the following properties hold:

$$
\begin{gathered}
|\nabla g(x)| \leq C \alpha \quad \text { for } w \text {-a.e. } x, \\
\operatorname{supp}\left(b_{i}\right) \subset B_{i} \quad \text { and } \quad \int_{B_{i}}\left|\nabla b_{i}\right|^{p} d w \leq C \alpha^{p} w\left(B_{i}\right), \\
\sum_{i} w\left(B_{i}\right) \leq \frac{C}{\alpha^{p}} \int_{\mathbb{R}^{n}}|\nabla f|^{p} d w \\
\sum_{i} \chi_{B_{i}} \leq N, \\
\left(f_{B_{i}}\left|b_{i}\right|^{q} d w\right)^{\frac{1}{q}} \lesssim C \alpha r\left(B_{i}\right) \quad \text { for } 1 \leq q \leq p_{w}^{*},
\end{gathered}
$$

where $C$ and $N$ depend only on $n, p, q$ and the doubling constant of $w$.

To prove (6.5) we will prove the corresponding weak-type estimates with $f$ replaced by $g$ and $b_{i}$. For $g$, we use the $L^{2}(w)$ Kato estimate (2.19), (6.7), and the fact that $p<2$ to get

$$
\begin{aligned}
w\left(\left\{\left|L_{w}^{1 / 2} g\right|>\alpha / 3\right\}\right) \lesssim \frac{1}{\alpha^{2}} & \int_{\mathbb{R}^{n}}\left|L_{w}^{1 / 2} g\right|^{2} d w \lesssim \frac{1}{\alpha^{2}} \int_{\mathbb{R}^{n}}|\nabla g|^{2} d w \lesssim \frac{1}{\alpha^{p}} \int_{\mathbb{R}^{n}}|\nabla g|^{p} d w \\
& \lesssim \frac{1}{\alpha^{p}} \int_{\mathbb{R}^{n}}|\nabla f|^{p} d w+\frac{1}{\alpha^{p}} \int_{\mathbb{R}^{n}}\left|\sum_{i} \nabla b_{i}\right|^{p} d w \lesssim \frac{1}{\alpha^{p}} \int_{\mathbb{R}^{n}}|\nabla f|^{p} d w
\end{aligned}
$$

where the last estimate follows from (6.10), (6.8), and (6.9).

To prove a weak-type estimate for $L_{w}^{1 / 2}\left(\sum_{i} b_{i}\right)$, let $r_{i}=2^{k}$ if $2^{k} \leq r\left(B_{i}\right)<2^{k+1}$. Then for all $i, r_{i} \sim r\left(B_{i}\right)$. Write

$$
L_{w}^{1 / 2}=\frac{1}{\sqrt{\pi}} \int_{0}^{r_{i}^{2}} L_{w} e^{-t L_{w}} \frac{d t}{\sqrt{t}}+\frac{1}{\sqrt{\pi}} \int_{r_{i}^{2}}^{\infty} L_{w} e^{-t L_{w}} \frac{d t}{\sqrt{t}}=T_{i}+U_{i} ;
$$

then we have that

$$
\begin{aligned}
w\left(\left\{\left|\sum_{i} L_{w}^{1 / 2} b_{i}\right|>\frac{2 \alpha}{3}\right\}\right) \leq w\left(\bigcup_{i} 4 B_{i}\right)+w\left(\left\{\left|\sum_{i} U_{i} b_{i}\right|>\frac{\alpha}{3}\right\}\right) \\
+w\left(\left(\mathbb{R}^{n} \backslash \bigcup_{i} 4 B_{i}\right) \bigcap\left\{\left|\sum_{i} T_{i} b_{i}\right|>\frac{\alpha}{3}\right\}\right)
\end{aligned}
$$




$$
\lesssim \frac{1}{\alpha^{p}} \int_{\mathbb{R}^{n}}|\nabla f|^{p} d w+I_{1}+I_{2}
$$

where the last inequality follows from (6.9).

We first estimate $I_{2}$. Since $p>\left(p_{-}\right)_{w, *}$ then $p_{w}^{*}>\left(\left(p_{-}\right)_{w, *}\right)_{w}^{*}=p_{-}$, and we can choose $q \in \mathcal{J}\left(L_{w}\right)$ such that $(6.11)$ is satisfied. By Corollary 3.4, $t L_{w} e^{-t L_{w}} \in \mathcal{O}\left(L^{q}(w) \rightarrow L^{q}(w)\right)$, and so

$$
\begin{aligned}
I_{2} & \lesssim \frac{1}{\alpha} \sum_{i} \sum_{j \geq 2} \int_{C_{j}\left(B_{i}\right)}\left|T_{i} b_{i}\right| d w \\
& \lesssim \frac{1}{\alpha} \sum_{i} \sum_{j \geq 2} w\left(2^{j} B_{i}\right) \int_{0}^{r_{i}^{2}} f_{C_{j}\left(B_{i}\right)}\left|t L_{w} e^{-t L_{w}} b_{i}\right| d w \frac{d t}{t^{3 / 2}} \\
& \lesssim \frac{1}{\alpha} \sum_{i} \sum_{j \geq 2} 2^{j D} w\left(B_{i}\right) \int_{0}^{r_{i}^{2}} 2^{j \theta_{1}} \Upsilon\left(\frac{2^{j} r_{i}}{\sqrt{t}}\right)^{\theta_{2}} e^{-\frac{c 4^{j} r_{i}^{2}}{t}} \frac{d t}{t^{3 / 2}}\left(f_{B_{i}}\left|b_{i}\right|^{q} d w\right)^{\frac{1}{q}} \\
& \lesssim \sum_{i} \sum_{j \geq 2} 2^{j D} e^{-c 4^{j}} w\left(B_{i}\right) \\
& \lesssim \sum_{i} w\left(B_{i}\right) \\
& \lesssim \frac{1}{\alpha^{p}} \int_{\mathbb{R}^{n}}|\nabla f|^{p} d w
\end{aligned}
$$

where we have used (6.11) and (6.9), and $D$ is the doubling order of $d w$.

We will now estimate $I_{1}$. For $q$ as above, by Proposition 4.3 we have an $L^{q}(w)$ functional calculus for $L_{w}$. Therefore, we can write $U_{i}$ as $r_{i}^{-1} \psi\left(r_{i}^{2} L_{w}\right)$ with $\psi$ defined by (5.15). Let $\beta_{k}=\sum_{i: r_{i}=2^{k}} \frac{b_{i}}{r_{i}}$; then,

$$
\sum_{i} U_{i} b_{i}=\sum_{k \in \mathbb{Z}} \psi\left(4^{k} L_{w}\right)\left(\sum_{i: r_{i}=2^{k}} \frac{b_{i}}{r_{i}}\right)=\sum_{k \in \mathbb{Z}} \psi\left(4^{k} L_{w}\right) \beta_{k} .
$$

Therefore, by Proposition 5.14, (6.10), (6.11), the fact that $r_{i} \sim r\left(B_{i}\right)$ and (6.9), we have that

$$
\begin{aligned}
I_{1} \lesssim \frac{1}{\alpha^{q}}\left\|\sum_{i} U_{i} b_{i}\right\|_{L^{q}(w)}^{q} \lesssim \frac{1}{\alpha^{q}} \| & \left(\sum_{k \in \mathbb{Z}}\left|\beta_{k}\right|^{2}\right)^{\frac{1}{2}} \|_{L^{q}(w)}^{q} \\
& \lesssim \frac{1}{\alpha^{q}} \int_{\mathbb{R}^{n}} \sum_{i} \frac{\left|b_{i}\right|^{q}}{r_{i}^{q}} d w \lesssim \sum_{i} w\left(B_{i}\right) \lesssim \frac{1}{\alpha^{p}} \int_{\mathbb{R}^{n}}|\nabla f|^{p} d w .
\end{aligned}
$$

If we combine all of the estimates we have obtained, we get (6.5) as desired.

To prove (6.2) from the weak-type estimate (6.5) we will use an interpolation argument from [5]. Fix $p$ and $r$ such that $\max \left\{r_{w},\left(p_{-}\right)_{w, *}\right\}<r<p<2$. Then by (6.5) and (2.19) we have that for every $f \in \mathcal{S}$,

$$
\left\|L_{w}^{1 / 2} f\right\|_{L^{r, \infty}(w)} \lesssim\|\nabla f\|_{L^{r}(w)}, \quad\left\|L_{w}^{1 / 2} f\right\|_{L^{2}(w)} \lesssim\|\nabla f\|_{L^{2}(w)}
$$

Formally, to apply Marcinkiewicz interpolation, we let $g=\nabla f$ to get a weak $(r, r)$ and strong $(2,2)$ inequality; this would immediately yield a strong $(p, p)$ inequality. To formalize this we must justify this substitution. 
For every $q>r_{w}$ by [5, Lemma 6.7] we have that

$$
\mathcal{E}=\left\{(-\Delta)^{1 / 2} f: f \in \mathcal{S}, \operatorname{supp} \widehat{f} \subset \mathbb{R}^{n} \backslash\{0\}\right\}
$$

is dense in $L^{q}(w)$, where $\widehat{f}$ denotes the Fourier transform of $f$. Moreover, since $r>r_{w}$, $w \in A_{r}$ and the Riesz transforms, $R_{j}=\partial_{j}(-\Delta)^{-1 / 2}$, are bounded on $L^{r}(w)$ [22]. It follows from this and the identity $-I=R_{1}^{2}+\cdots+R_{n}^{2}$ that for $g \in L^{r}(w)$,

$$
\|g\|_{L^{r}(w)} \sim\left\|\nabla(-\Delta)^{-1 / 2} g\right\|_{L^{r}(w)} .
$$

Thus, for $g \in \mathcal{E}, L_{w}^{1 / 2}(-\Delta)^{-1 / 2} g=L_{w}^{1 / 2} f$ if $f=(-\Delta)^{-1 / 2} g$ and $\|\nabla f\|_{L^{r}(w)} \sim\|g\|_{L^{r}(w)}$ for $r>r_{w}$. Thus (6.12) becomes weighted weak $(r, r)$ and strong $(2,2)$ inequalities for $T=L_{w}^{1 / 2}(-\Delta)^{-1 / 2}$, and this operator is defined a priori on $\mathcal{E}$. Since $\mathcal{E}$ is dense in each $L^{q}(w)$, we can extend $T$ by density in both cases and their restrictions to the space of simple functions agree. Hence, we can apply Marcinkiewicz interpolation and conclude, again by density, that (6.2) holds for all $p$ with $r<p<2$. Since $r$ is arbitrary, we get (6.2) in the range $\max \left\{r_{w},\left(p_{-}\right)_{w, *}\right\}<p<2$.

For the second step of the proof we will prove (6.3) using Theorem 2.35. Inequality (6.2) for its full range of exponents then follows by letting $v=1$. Define $\tilde{p}_{-}=\max \left\{r_{w}, p_{-}\right\}<2$, and fix $\tilde{p}_{-}<p<p_{+}$and $v \in A_{p / \tilde{p}_{-}}(w) \cap R H_{\left(p_{+} / p\right)^{\prime}}(w)$. By the openness properties of $A_{q}$ and $R H_{s}$ weights, there exist $p_{0}, q_{0}$ such that

$$
\tilde{p}_{-}<p_{0}<\min \{p, 2\} \leq p<q_{0}<p_{+}, \quad v \in A_{p / p_{0}}(w) \cap R H_{\left(q_{0} / p\right)^{\prime}}(w) .
$$

To apply Theorem 2.35 , let $T=L_{w}^{1 / 2}, S=\nabla$, and $\mathcal{A}_{r}=I-\left(I-e^{-r^{2} L_{w}}\right)^{m}$ where the value of $m$ will be fixed below. We will first show that (2.37) holds. By (4.8) we have that $\mathcal{A}_{r} \in \mathcal{O}\left(L^{p_{0}}(w) \rightarrow L^{q_{0}}(w)\right)$ since $p_{0}, q_{0} \in \mathcal{J}\left(L_{w}\right)$. Let $h=L_{w}^{1 / 2} f$ and decompose $h$ as we decomposed $f$ in (4.11). Then, since $L_{w}^{1 / 2}$ and $\mathcal{A}_{r}$ commute, it follows that

$$
\begin{aligned}
\left(f_{B}\left|L_{w}^{1 / 2} \mathcal{A}_{r} f\right|^{q_{0}} d w\right)^{\frac{1}{q_{0}}} & \lesssim \sum_{j \geq 1}\left(f_{B}\left|\mathcal{A}_{r} h_{j}\right|^{q_{0}} d w\right)^{\frac{1}{q_{0}}} \\
& \lesssim \sum_{j \geq 1} 2^{j \theta_{1}} \Upsilon\left(2^{j}\right)^{\theta_{2}} e^{-c 4^{j}}\left(f_{C_{j}}|h|^{p_{0}} d w\right)^{\frac{1}{p_{0}}} \\
& \leq \sum_{j \geq 1} 2^{j\left(\theta_{1}+\theta_{2}\right)} e^{-c 4^{j}}\left(f_{2^{j+1} B}\left|L_{w}^{1 / 2} f\right|^{p_{0}} d w\right)^{\frac{1}{p_{0}}}
\end{aligned}
$$

This gives us (2.37) with $g(j)=C 2^{j\left(\theta_{1}+\theta_{2}\right)} e^{-c 4^{j}}$; clearly, $\sum g(j)<\infty$.

We now prove that $(2.36)$ holds. Fix $f \in \mathcal{S}$ and let $\varphi(z)=z^{1 / 2}\left(1-e^{-r^{2} z}\right)^{m}$ so that $\varphi\left(L_{w}\right) f=L_{w}^{1 / 2}\left(I-e^{-r^{2} L_{w}}\right)^{m} f$. By the conservation property (see [19] or [2, Section 2.5]),

$$
\varphi\left(L_{w}\right) f=\varphi\left(L_{w}\right)\left(f-f_{4 B, w}\right)=\sum_{j \geq 1} \varphi\left(L_{w}\right) h_{j}
$$

where $h_{j}=\left(f-f_{4 B, w}\right) \phi_{j}, \phi_{j}=\chi_{C_{j}(B)}$ for $j \geq 3, \phi_{1}$ is a smooth function with support in $4 B, 0 \leq \phi_{1} \leq 1, \phi_{1}=1$ in $2 B$ and $\left\|\nabla \phi_{1}\right\|_{\infty} \leq C / r$, and $\phi_{2}$ is chosen so that $\sum_{j \geq 1} \phi_{j}=1$. 
We estimate each term in the righthand side of (6.13) separately. When $j=1$, since $p_{-}<p_{0}<p_{+}$, by the bounded holomorphic functional calculus on $L^{p_{0}}(w)$ (Proposition 4.3) and the fact that $\varphi\left(L_{w}\right) h_{1}=\left(I-e^{-r^{2} L_{w}}\right)^{m} L_{w}^{1 / 2} h_{1}$, we have that

$$
\left\|\varphi\left(L_{w}\right) h_{1}\right\|_{L^{p_{0}(w)}} \lesssim\left\|L_{w}^{1 / 2} h_{1}\right\|_{L^{p_{0}}(w)}
$$

uniformly in $r$. By the above argument we have that (6.2) holds for $p=p_{0}$ since $\tilde{p}_{-}<p_{0}<2$. Further, since $f \in \mathcal{S}, h_{1} \in \mathcal{S}$ by our choice of $\phi_{1}$. This, together with the $L^{p_{0}}(w)$-Poincaré inequality (2.3) (since $p_{0}>r_{w}, w \in A_{p_{0}}$ ) and the definition of $h_{1}$ yield

$$
\begin{aligned}
\left\|L_{w}^{1 / 2} h_{1}\right\|_{L^{p_{0}}(w)} & \lesssim\left\|\nabla h_{1}\right\|_{L^{p_{0}}(w)} \\
& \lesssim\left\|(\nabla f) \chi_{4 B}\right\|_{L^{p_{0}}(w)}+r^{-1}\left\|\left(f-f_{4 B, w}\right) \chi_{4 B}\right\|_{L^{p_{0}(w)}} \lesssim\left\|(\nabla f) \chi_{4 B}\right\|_{L^{p_{0}}(w)} .
\end{aligned}
$$

Therefore,

$$
\left(f_{B}\left|\varphi\left(L_{w}\right) h_{1}\right|^{p_{0}} d w\right)^{\frac{1}{p_{0}}} \lesssim\left(f_{4 B}|\nabla f|^{p_{0}} d w\right)^{\frac{1}{p_{0}}} .
$$

When $j \geq 3$, the functions $\eta$ associated with $\varphi$ by (2.11) satisfy

$$
|\eta(z)| \lesssim \frac{r^{2 m}}{|z|^{m+3 / 2}}, \quad z \in \Gamma_{\pi / 2-\theta}
$$

Since $p_{0} \in \mathcal{J}\left(L_{w}\right)$, by Corollary $3.4, e^{-z L_{w}} \in \mathcal{O}\left(L^{p_{0}}(w) \rightarrow L^{p_{0}}(w), \Sigma_{\mu}\right)$. This, together with the representation $(2.10)$, gives us that

$$
\begin{aligned}
\left(f_{B} \mid \varphi\right. & \left.\left.\left(L_{w}\right) h_{j}\right|^{p_{0}} d w\right)^{\frac{1}{p_{0}}} \\
& \leq \int_{\Gamma_{\pi / 2-\theta}}\left(f_{B}\left|e^{-z L} h_{j}\right|^{p_{0}} d w\right)^{\frac{1}{p_{0}}}|\eta(z)||d z| \\
& \lesssim 2^{j \theta_{1}} \int_{\Gamma_{\pi / 2-\theta}} \Upsilon\left(\frac{2^{j} r}{\sqrt{|z|}}\right)^{\theta_{2}} e^{-\frac{\alpha 4^{j} r^{2}}{|z|}} \frac{r^{2 m}}{|z|^{m+3 / 2}}|d z|\left(f_{C_{j}(B)}\left|h_{j}\right|^{p_{0}} d w\right)^{\frac{1}{p_{0}}} \\
& \lesssim 2^{j\left(\theta_{1}-2 m-1\right)} r^{-1}\left(f_{2^{j+1} B}\left|f-f_{4 B, w}\right|^{p_{0}} d w\right)^{\frac{1}{p_{0}}} \\
& \lesssim 2^{j\left(\theta_{1}-2 m-1\right)} \sum_{l=1}^{j} 2^{l}\left(f_{2^{l+1} B}|\nabla f|^{p_{0}} d x\right)^{\frac{1}{p_{0}}}
\end{aligned}
$$

provided $2 m+1>\theta_{2}$. The last estimate follows from $L^{p_{0}}(w)$-Poincaré inequality (2.3) (here we again use that $p_{0}>r_{w}$ and so $\left.w \in A_{p_{0}}\right)$ :

$$
\begin{aligned}
\left(f_{2^{j+1} B}\right. & \left.\left|f-f_{4 B, w}\right|^{p_{0}} d w\right)^{\frac{1}{p_{0}}} \\
& \leq\left(f_{2^{j+1} B}\left|f-f_{2^{j+1} B, w}\right|^{p_{0}} d w\right)^{\frac{1}{p_{0}}}+\sum_{l=2}^{j}\left|f_{2^{l} B, w}-f_{2^{l+1} B, w}\right| \\
& \lesssim \sum_{l=1}^{j}\left(f_{2^{l+1} B}\left|f-f_{2^{l+1} B}\right|^{p_{0}} d x\right)^{\frac{1}{p_{0}}}
\end{aligned}
$$




$$
\lesssim r \sum_{l=1}^{j} 2^{l}\left(f_{2^{l+1} B}|\nabla f|^{p_{0}} d x\right)^{\frac{1}{p_{0}}}
$$

When $j=2$ we can argue similarly, using the fact that

$$
\left|h_{2}\right| \leq\left|f-f_{4 B, w}\right| \chi_{8 B \backslash 2 B} \leq\left|f-f_{2 B, w}\right| \chi_{8 B \backslash 2 B}+\left|f_{4 B, w}-f_{2 B, w}\right| \chi_{8 B \backslash 2 B} .
$$

If we combine these estimates, then by (6.13) and Minkowski's inequality we get

$$
\left(f_{B}\left|\varphi\left(L_{w}\right) h\right|^{p_{0}} d w\right)^{\frac{1}{p_{0}}} \lesssim \sum_{j \geq 1}\left(f_{B}\left|\varphi\left(L_{w}\right) h_{j}\right|^{p_{0}} d w\right)^{\frac{1}{p_{0}}} \leq \sum_{j \geq 1} g(j)\left(f_{B}|\nabla f|^{p_{0}} d w\right)^{\frac{1}{p_{0}}}
$$

with $g(j)=C_{m} 2^{j\left(\theta_{1}-2 m\right)}$ provided $2 m+1>\theta_{2}$. If we further assume that $2 m>\theta_{1}$, then $\sum_{j} g(j)<\infty$. This proves that (2.36) holds. Therefore, by Theorem 2.35 we get (6.3) as desired.

\section{The GRAdient of The SEMigroup $\sqrt{t} \nabla e^{-t L_{w}}$}

Let $\widetilde{\mathcal{K}}\left(L_{w}\right) \subset[1, \infty]$ be the set of all exponents $p$ such that $\sqrt{t} \nabla e^{-t L_{w}}: L^{p}(w) \rightarrow L^{p}(w)$ is uniformly bounded for all $t>0$. By Theorem 2.15 and Lemma $2.34,2 \in \widetilde{\mathcal{K}}\left(L_{w}\right)$ and if it contains more than one point, then by interpolation $\widetilde{\mathcal{K}}\left(L_{w}\right)$ is an interval. In this section we give a partial description of the set of $(p, q)$ such that $\sqrt{t} \nabla e^{-t L_{w}} \in \mathcal{O}\left(L^{p}(w) \rightarrow L^{q}(w)\right)$.

Proposition 7.1. There exists an interval $\mathcal{K}\left(L_{w}\right)$ such that if $p, q \in \mathcal{K}\left(L_{w}\right), p \leq q$, then $\sqrt{t} \nabla e^{-t L_{w}} \in \mathcal{O}\left(L^{p}(w) \rightarrow L^{q}(w)\right)$. Moreover, $\mathcal{K}\left(L_{w}\right)$ has the following properties:

(1) $\mathcal{K}\left(L_{w}\right) \subset \widetilde{\mathcal{K}}\left(L_{w}\right)$;

(2) if $q_{-}\left(L_{w}\right)$ and $q_{+}\left(L_{w}\right)$ are the left and right endpoints of $\mathcal{K}\left(L_{w}\right)$, then $q_{-}\left(L_{w}\right)=$ $p_{-}\left(L_{w}\right), 2 \leq q_{+}\left(L_{w}\right) \leq\left(q_{+}\left(L_{w}\right)\right)_{w}^{*} \leq p_{+}\left(L_{w}\right)$. In particular, $2 \in \mathcal{K}\left(L_{w}\right)$ and $\mathcal{K}\left(L_{w}\right) \subset$ $\mathcal{J}\left(L_{w}\right)$;

(3) If $q \geq 2$ and $p<q$, and if $\sqrt{t} \nabla e^{-t L_{w}} \in \mathcal{O}\left(L^{p}(w) \rightarrow L^{q}(w)\right)$, then $p, q \in \mathcal{K}\left(L_{w}\right)$;

(4) $\sup \widetilde{\mathcal{K}}\left(L_{w}\right)=q_{+}\left(L_{w}\right)$.

Remark 7.2. Unlike in the unweighted case (see [7]) we are unable to give a complete characterization of $\mathcal{K}\left(L_{w}\right)$. More precisely, if we have an off-diagonal estimate and $p<q<2$, then we cannot prove that $p, q \in \mathcal{K}\left(L_{w}\right)$.

Remark 7.3. In Section 8 below we will show that $q_{+}\left(L_{w}\right)>2$; in particular, this gives that $2 \in \operatorname{Int} \mathcal{K}\left(L_{w}\right)$.

As an immediate consequence of Proposition 7.1 we get weighted inequalities for the gradient of the semigroup. The proof is identical to the proof of Corollaries 3.3 and 3.4.

Corollary 7.4. Let $q_{-}\left(L_{w}\right)<p \leq q<q_{+}\left(L_{w}\right)$. If $v \in A_{p / q_{-}\left(L_{w}\right)}(w) \cap R H_{\left(q_{+}\left(L_{w}\right) / q\right)^{\prime}}(w)$, then $\sqrt{t} \nabla e^{-t L_{w}} \in \mathcal{O}\left(L^{p}(v d w) \rightarrow L^{q}(v d w)\right)$ and $\sqrt{z} \nabla e^{-z L_{w}} \in \mathcal{O}\left(L^{p}(v d w) \rightarrow L^{q}(v d w), \Sigma_{\nu}\right)$ for all $\nu, 0<\nu<\frac{\pi}{2}-\vartheta$.

The proof of Proposition 7.1 requires two lemmas. 
Lemma 7.5. Given $w \in A_{\infty}$ and a family of sublinear operators $\left\{T_{t}\right\}_{t>0}$ such that $T_{t} \in$ $\mathcal{O}\left(L^{p}(w) \rightarrow L^{q}(w)\right)$, with $1 \leq p<q \leq \infty$, there exist $\alpha, \beta>0$ such that for any ball $B$ with radius $r$ and for any $t>0$,

$$
\left(f_{B}\left|T_{t}\left(\chi_{B} f\right)\right|^{q} d w\right)^{\frac{1}{q}} \lesssim \max \left\{\left(\frac{r}{\sqrt{t}}\right)^{\alpha},\left(\frac{r}{\sqrt{t}}\right)^{\beta}\right\}\left(f_{B}|f|^{p} d w\right)^{\frac{1}{p}} .
$$

Proof. This result is implicit in [7, Proof of Proposition 2.4, p. 306]; here we reprove it with a small improvement in the constant. There it was shown that in Definition 2.23 it is sufficient to consider the case where $r \approx \sqrt{t}$. But in this case we get that $\Upsilon(r / \sqrt{t}) \approx 1$ and for all $j \geq 2, \Upsilon\left(2^{j} r / \sqrt{t}\right) \approx 2^{j}$. The argument in [7, p. 306] shows that if we assume that $(2.24),(2.25),(2.26)$ hold when $r \approx \sqrt{t}$, then (2.24) holds in general with constant $\max \left\{1,(r / \sqrt{t})^{\alpha}\right\}$ for some $\alpha>0$ depending on $p, q$ and $w$. In this maximum the 1 occurs when $r \leq \sqrt{t}$; therefore, to prove (7.6) we need to show that if $r \leq \sqrt{t}$, then we can replace 1 by the better constant $(r / \sqrt{t})^{\beta}$ for some $\beta>0$.

Fix $r \leq \sqrt{t}$. If $B=B(x, r)$, then $B \subset B_{t}=B(x, \sqrt{t})$. As in [7, p. 306] we apply (2.24) to $T_{t}$ and $B_{t}$; this yields

$$
\begin{aligned}
\left(f_{B}\left|T_{t}\left(\chi_{B} f\right)\right|^{q} d w\right)^{\frac{1}{q}} & \leq\left(\frac{w\left(B_{t}\right)}{w(B)}\right)^{\frac{1}{q}}\left(f_{B_{t}}\left|T_{t}\left(\chi_{B} f\right)\right|^{q} d w\right)^{\frac{1}{q}} \\
& \lesssim\left(\frac{w\left(B_{t}\right)}{w(B)}\right)^{\frac{1}{q}}\left(f_{B_{t}}\left|\chi_{B} f\right|^{p} d w\right)^{\frac{1}{p}} \leq\left(\frac{w(B)}{w\left(B_{t}\right)}\right)^{\frac{1}{p}-\frac{1}{q}}\left(f_{B}|f|^{p} d w\right)^{\frac{1}{p}} .
\end{aligned}
$$

Since $w \in A_{\infty}$, we have that for some $\theta>0$,

$$
\frac{w(B)}{w\left(B_{t}\right)} \lesssim\left(\frac{|B|}{\left|B_{t}\right|}\right)^{\theta}=\left(\frac{r}{\sqrt{t}}\right)^{\theta n}
$$

Since $p<q$ we have that

$$
\left(f_{B}\left|T_{t}\left(\chi_{B} f\right)\right|^{q} d w\right)^{\frac{1}{q}} \lesssim\left(\frac{r}{\sqrt{t}}\right)^{\left(\frac{1}{p}-\frac{1}{q}\right) \theta n}\left(f_{B}|f|^{p} d w\right)^{\frac{1}{p}}
$$

Therefore, if we combine this with the argument from [7, p. 306] described above, we get that (7.6) holds with $\beta=(1 / p-1 / q) \theta n$.

The second lemma gives the close connection between off-diagonal estimates for $e^{-t L_{w}}$ and $\sqrt{t} \nabla e^{-t L_{w}}$ for $p<2$.

Lemma 7.7. Given $1 \leq p<2$ the following are equivalent:

(1) $e^{-t L_{w}} \in \mathcal{O}\left(L^{p}(w) \rightarrow L^{2}(w)\right)$.

(2) $\sqrt{t} \nabla e^{-t L_{w}} \in \mathcal{O}\left(L^{p}(w) \rightarrow L^{2}(w)\right)$.

(3) $t L_{w} e^{-t L_{w}} \in \mathcal{O}\left(L^{p}(w) \rightarrow L^{2}(w)\right)$.

Proof. We follow the proof of [7, Lemma 5.3]. To prove that (1) implies (2), note that by Theorem 2.15, $\sqrt{t} \nabla e^{-t L_{w}} \in \mathcal{O}\left(L^{2}(w) \rightarrow L^{2}(w)\right)$. If we compose this with (1), by Lemma 2.30 and the semigroup property, we get (2).

To prove that (2) implies (3), define $S_{t} \vec{f}=\sqrt{t} e^{-t L_{w}}\left(w^{-1} \operatorname{div}(A \vec{f})\right)$. By duality, we have that 


$$
\begin{aligned}
\left\langle S_{t} \vec{f}, g\right\rangle_{L^{2}(w)}=\left\langle w^{-1} \operatorname{div}(A \vec{f})\right), & \left.\left.\sqrt{t} e^{-t L_{w}^{*}} g\right\rangle_{L^{2}(w)}=\langle\operatorname{div}(A \vec{f})), \sqrt{t} e^{-t L_{w}^{*}} g\right\rangle_{L^{2}} \\
& =-\left\langle\vec{f}, A^{*} \sqrt{t} \nabla e^{-t L_{w}^{*}} g\right\rangle_{L^{2}}=\left\langle\vec{f}, w^{-1} A^{*} \sqrt{t} \nabla e^{-t L_{w}^{*}} g\right\rangle_{L^{2}(w)} .
\end{aligned}
$$

The matrix $w^{-1} A^{*}$ is uniformly elliptic, and so multiplication by it is bounded on $L^{2}(w)$. Furthermore, $\sqrt{t} \nabla e^{-t L_{w}^{*}} \in \mathcal{O}\left(L^{2}(w) \rightarrow L^{2}(w)\right)$. Therefore, it follows that $S_{t} \in \mathcal{O}\left(L^{2}(w) \rightarrow\right.$ $\left.L^{2}(w)\right)$. If we combine this with (2), we get that $-t L_{w} e^{-2 t L_{w}}=S_{t} \circ \sqrt{t} \nabla e^{-t L_{w}} \in$ $\mathcal{O}\left(L^{p}(w) \rightarrow L^{2}(w)\right)$. This proves $(3)$.

Finally we show that (3) implies (1). We first prove (2.24). Fix $B$ and $f, g$ such that $\left(f_{B}|f|^{p} d w\right)^{\frac{1}{p}}=\left(f_{B}|g|^{2} d w\right)^{\frac{1}{2}}=1$, and assume also that $f \in L^{2}(B, d w)$. Define

$$
h(t)=f_{B} e^{-t L_{w}}\left(\chi_{B} f\right)(x) g(x) d w(x) .
$$

By duality it will suffice to show that $|h(t)| \lesssim \Upsilon(r / \sqrt{t})^{\theta}$. (Note that our assumption implies that $t h^{\prime}(t)$ satisfies such a bound.) First, we claim that

$$
\lim _{t \rightarrow \infty} h(t)=0 .
$$

To see this we use the fact (discussed in Section 2) that $L_{w}$ has a bounded holomorphic functional calculus on $L^{2}(w)$. Given this, since $z \mapsto e^{-t z}$ converges to 0 uniformly on compact subsets of $\operatorname{Re} z>0$, we get the desired limit.

Hence, we can write $h(t)=-\int_{t}^{\infty} h^{\prime}(s) d s$. Notice that $\left|t h^{\prime}(t)\right| \lesssim \Upsilon(r / \sqrt{t})^{\theta_{2}}$ but this does not give a convergent integral. However, if we apply Lemma 7.5 to $t L_{w} e^{-t L_{w}} \in \mathcal{O}\left(L^{p}(w) \rightarrow\right.$ $\left.L^{2}(w)\right)$, we get that $\left|t h^{\prime}(t)\right| \lesssim \widetilde{\Upsilon}(r / \sqrt{t})$ with $\widetilde{\Upsilon}(s)=\max \left\{s^{\alpha}, s^{\beta}\right\}$. It follows from this estimate that

$$
|h(t)| \leq \int_{t}^{\infty}\left|h^{\prime}(s)\right| d s \lesssim \int_{t}^{\infty} \widetilde{\Upsilon}\left(\frac{r}{\sqrt{s}}\right) \frac{d s}{s} \approx \int_{0}^{\frac{r}{\sqrt{t}}} \widetilde{\Upsilon}(s) \frac{d s}{s} \lesssim \widetilde{\Upsilon}\left(\frac{r}{\sqrt{t}}\right) \lesssim \Upsilon\left(\frac{r}{\sqrt{t}}\right)^{\alpha+\beta} .
$$

To prove (2.25) we argue as before, but with $\left(f_{C_{j}(B)}|f|^{p} d w\right)^{\frac{1}{p}}=\left(f_{B}|g|^{2} d w\right)^{\frac{1}{2}}=1$ and

$$
h(t)=f_{B} e^{-t L_{w}}\left(\chi_{C_{j}(B)} f\right)(x) g(x) d w(x) .
$$

Since $d\left(B, C_{j}(B)\right)>0$, by Theorem 2.15 and Hölder's inequality, $h(t) \rightarrow 0$ as $t \rightarrow 0$. Therefore, $h(t)=\int_{0}^{t} h^{\prime}(s) d s$. Since $t L_{w} e^{-t L_{w}} \in \mathcal{O}\left(L^{p}(w) \rightarrow L^{2}(w)\right)$, we have that

$$
\begin{aligned}
h(t) \leq \int_{0}^{t}\left|h^{\prime}(s)\right| d s \lesssim 2^{j \theta_{1}} \int_{0}^{t} \Upsilon( & \left.\frac{2^{j} r}{\sqrt{s}}\right)^{\theta_{2}} e^{-\frac{c 4^{j} r^{2}}{s}} \frac{d s}{s} \\
& \approx 2^{j \theta_{1}} \int_{\frac{2^{j} r}{\sqrt{t}}}^{\infty} \Upsilon(s)^{\theta_{2}} e^{-c s^{2}} \frac{d s}{s} \lesssim 2^{j \theta_{1}} \Upsilon\left(\frac{2^{j} r}{\sqrt{t}}\right)^{\theta_{2}} e^{-\frac{c 4^{j} r^{2}}{t}} .
\end{aligned}
$$

This is (2.25).

Finally, the proof of (2.26) is essentially the same and we omit the details. This completes the proof that (3) implies (1).

Proof of Proposition 7.1. Define the sets $\mathcal{K}_{-}\left(L_{w}\right)$ and $\mathcal{K}_{+}\left(L_{w}\right)$ to be

$$
\mathcal{K}_{-}\left(L_{w}\right)=\left\{p \in[1,2]: \sqrt{t} \nabla e^{-t L_{w}} \in \mathcal{O}\left(L^{p}(w) \rightarrow L^{2}(w)\right)\right\}
$$




$$
\mathcal{K}_{+}\left(L_{w}\right)=\left\{p \in[2, \infty]: \sqrt{t} \nabla e^{-t L_{w}} \in \mathcal{O}\left(L^{2}(w) \rightarrow L^{p}(w)\right)\right\},
$$

and let $\mathcal{K}\left(L_{w}\right)=\mathcal{K}_{-}\left(L_{w}\right) \cup \mathcal{K}_{+}\left(L_{w}\right)$. The set is non-empty, since $2 \in \mathcal{K}\left(L_{w}\right)$. By Lemma 2.28 it is an interval. Now fix $p, q \in \mathcal{K}\left(L_{w}\right)$ with $p<q$. If $p<q \leq 2$ or $2 \leq p<q$, then by Lemma 2.28, $\sqrt{t} \nabla e^{-t L} \in \mathcal{O}\left(L^{p}(w) \rightarrow L^{q}(w)\right)$ since $p, q \in \mathcal{K}_{-}\left(L_{w}\right)$ or $p, q \in \mathcal{K}_{+}\left(L_{w}\right)$. If $p \leq$ $2<q$, then $\sqrt{t} \nabla e^{-t L} \in \mathcal{O}\left(L^{2}(w) \rightarrow L^{q}(w)\right)$ and by Lemma 7.7, $e^{-t L} \in \mathcal{O}\left(L^{p}(w) \rightarrow L^{2}(w)\right)$. Hence, by Lemma 2.30 and the semigroup property, $\sqrt{t} \nabla e^{-t L} \in \mathcal{O}\left(L^{p}(w) \rightarrow L^{q}(w)\right)$. Thus, in every case we get the desired off-diagonal estimate.

We now prove (1)-(4). By Lemma 2.30, off-diagonal estimates on balls imply uniform boundedness, and so $\mathcal{K}\left(L_{w}\right) \subset \widetilde{\mathcal{K}}\left(L_{w}\right)$. This proves $(1)$.

To prove (2), we first note that if $p<2$, then by Lemma 7.7, $p \in \mathcal{J}\left(L_{w}\right)$ if and only if $p \in$ $\mathcal{K}_{-}\left(L_{w}\right)$. Thus $\mathcal{J}\left(L_{w}\right) \cap[1,2]=\mathcal{K}_{-}\left(L_{w}\right)$ and so $q_{-}\left(L_{w}\right)=p_{-}\left(L_{w}\right)$. To show that $\left(q_{+}\left(L_{w}\right)\right)_{w}^{*} \leq$ $p_{+}\left(L_{w}\right)$, first note that if $q_{+}\left(L_{w}\right)=2$, then by Proposition 3.1 we have that $\left(q_{+}\left(L_{w}\right)\right)_{w}^{*}=$ $2_{w}^{*} \leq p_{+}\left(L_{w}\right)$. If $q_{+}\left(L_{w}\right)>2$, then we proceed as in the proof of this proposition. Let $2<p<q_{+}\left(L_{w}\right)$ and $p<q<p_{w}^{*}$. Then by $(2.3)$, $e^{-t L_{w}} \in \mathcal{O}\left(L^{2}(w) \rightarrow L^{2}(w)\right)$, and $\sqrt{t} \nabla e^{-t L_{w}} \in \mathcal{O}\left(L^{2}(w) \rightarrow L^{p}(w)\right)$, we get that

$$
\begin{aligned}
& \left(f_{B}\left|e^{-t L_{w}}\left(\chi_{B} f\right)\right|^{q} d w\right)^{\frac{1}{q}} \\
& \quad \lesssim\left(f_{B}\left|e^{-t L_{w}}\left(\chi_{B} f\right)\right|^{2} d w\right)^{\frac{1}{2}}+r\left(f_{B}\left|\nabla e^{-t L_{w}}\left(\chi_{B} f\right)\right|^{p} d w\right)^{\frac{1}{p}} \\
& \quad \lesssim \Upsilon\left(\frac{r}{\sqrt{t}}\right)^{1+\theta_{2}}\left(f_{B}|f|^{2} d w\right)^{\frac{1}{2}} .
\end{aligned}
$$

This gives us inequality (2.24). The other two inequalities in Definition 2.23 can be proved in exactly the same way. Thus $e^{-t L_{w}} \in \mathcal{O}\left(L^{2}(w) \rightarrow L^{q}(w)\right)$ which implies $q \leq p_{+}\left(L_{w}\right)$. Letting $p \nearrow q_{+}\left(L_{w}\right)$ and $q \nearrow p_{w}^{*}$ we conclude that $\left(q_{+}\left(L_{w}\right)\right)_{w}^{*} \leq p_{+}\left(L_{w}\right)$.

The last estimate implies in particular that $q_{+}\left(L_{w}\right) \leq p_{+}\left(L_{w}\right)$. If $q_{+}\left(L_{w}\right)<\infty$ we clearly have that $q_{+}\left(L_{w}\right)<p_{+}\left(L_{w}\right)$ and so $\mathcal{K}_{+}\left(L_{w}\right) \subset \mathcal{J}\left(L_{w}\right)$. Otherwise, $p_{+}(L)=\infty$ and again we have that $\mathcal{K}_{+}\left(L_{w}\right) \subset \mathcal{J}\left(L_{w}\right)$. This completes the proof of $(2)$.

To prove (3), suppose first that $2 \leq p<q$ and $\sqrt{t} \nabla e^{-t L} \in \mathcal{O}\left(L^{p}(w) \rightarrow L^{q}(w)\right)$. We will show that $p, q \in \mathcal{K}\left(L_{w}\right)$. Since we also have that $\sqrt{t} \nabla e^{-t L} \in \mathcal{O}\left(L^{2}(w) \rightarrow L^{2}(w)\right)$, by interpolation (Lemma 2.27), $\sqrt{t} \nabla e^{-t L} \in \mathcal{O}\left(L^{p_{\theta}}(w) \rightarrow L^{q_{\theta}}(w)\right)$ where $1 / p_{\theta}=(1-\theta) / p+\theta / 2$, $1 / q_{\theta}=(1-\theta) / q+\theta / 2$ and $\theta \in(0,1)$. If $p \notin \mathcal{K}_{+}\left(L_{w}\right)$, then $q>\sup \mathcal{K}_{+}\left(L_{w}\right)$. We can choose $\theta$ such that $p_{\theta}<\sup \mathcal{K}_{+}\left(L_{w}\right)<q_{\theta}$. Since $\mathcal{K}_{+}\left(L_{w}\right) \subset \mathcal{J}\left(L_{w}\right), p_{\theta} \in \mathcal{J}\left(L_{w}\right)$ : i.e., $e^{-t L} \in \mathcal{O}\left(L^{2}(w) \rightarrow L^{p_{\theta}}(w)\right)$. By composition and the semigroup property, $\sqrt{t} \nabla e^{-t L_{w}} \in$ $\mathcal{O}\left(L^{2}(w) \rightarrow L^{q_{\theta}}(w)\right)$; hence, $q_{\theta} \in \mathcal{K}_{+}\left(L_{w}\right)$, a contradiction. Therefore, $p \in \mathcal{K}_{+}\left(L_{w}\right)$. As we have $\sqrt{t} \nabla e^{-t L_{w}} \in \mathcal{O}\left(L^{p}(w) \rightarrow L^{q}(w)\right)$ by assumption and $e^{-t L_{w}} \in \mathcal{O}\left(L^{2}(w) \rightarrow L^{p}(w)\right)$ since $p \in \mathcal{J}\left(L_{w}\right)$, by composition and the semigroup property, $\sqrt{t} \nabla e^{-t L_{w}} \in \mathcal{O}\left(L^{2}(w) \rightarrow L^{q}(w)\right)$. Hence, $q \in \mathcal{K}_{+}\left(L_{w}\right)$.

The case $p<2 \leq q$ is straightforward. Since $\sqrt{t} \nabla e^{-t L_{w}} \in \mathcal{O}\left(L^{p}(w) \rightarrow L^{q}(w)\right)$, by Lemma 2.28 we have that $\sqrt{t} \nabla e^{-t L_{w}} \in \mathcal{O}\left(L^{2}(w) \rightarrow L^{q}(w)\right)$ and $\sqrt{t} \nabla e^{-t L_{w}} \in \mathcal{O}\left(L^{p}(w) \rightarrow\right.$ $\left.L^{2}(w)\right)$. Hence, $p \in \mathcal{K}_{-}\left(L_{w}\right)$ and $q \in \mathcal{K}_{+}\left(L_{w}\right)$. 
Finally, we prove (4). Suppose to the contrary that $\sup \widetilde{\mathcal{K}}\left(L_{w}\right)>q_{+}\left(L_{w}\right)$. Then there exist $p, q$ such that $q_{+}\left(L_{w}\right)<p<q<\sup \widetilde{\mathcal{K}}\left(L_{w}\right)$. Fix $r$ such that $p_{-}\left(L_{w}\right)=q_{-}\left(L_{w}\right)<r<2$. Then we have that $\sqrt{t} \nabla e^{-t L_{w}}$ is uniformly bounded on $L^{q}(w)$ and in $\mathcal{O}\left(L^{r}(w) \rightarrow L^{2}(w)\right)$. By Lemma 2.27 we can interpolate between these to get that $\sqrt{t} \nabla e^{-t L_{w}} \in \mathcal{O}\left(L^{s}(w) \rightarrow L^{p}(w)\right)$ for some $s<p$. But then by the above converse, we have that $p \in \mathcal{K}\left(L_{w}\right)$ which is a contradiction.

\section{AN UPPER BOUND FOR $\mathcal{K}\left(L_{w}\right)$}

In this section we will prove that $q_{+}\left(L_{w}\right)>2$ : that is, the set $\mathcal{K}\left(L_{w}\right)$ contains 2 in its interior. In general, all we can say is that $q_{+}\left(L_{w}\right)>2$ : as noted in [2, Section 4.5], even in the unweighted case this is the best possible bound, since given any $\epsilon>0$ it is possible to find an operator $L$ such that $q_{+}(L)<2+\epsilon$. In Section 11 below we will give some estimates for $q_{+}\left(L_{w}\right)$ in terms of $[w]_{A_{2}}$.

We have broken the proof that $q_{+}\left(L_{w}\right)>2$ into a series of discrete steps where we borrow some ideas from [3]. We first prove a reverse Hölder inequality and use Gehring's inequality to get a higher integrability estimate. We then prove that the Hodge projection is bounded on $L^{q}(w)$ for a range of $q>2$ and use this to prove the Riesz transform is also bounded for exponents greater than 2. (In Section 9 we give a more complete discussion of the Riesz transform.) From this we deduce that $q_{+}\left(L_{w}\right)>2$.

A reverse Hölder inequality. Fix a ball $B_{0}$ and let $u \in H^{1}(w)$ be any weak solution of $L_{w} u=0$ in $4 B_{0}$. Then for any ball $B$ such that $3 B \subset 4 B_{0}$, we can again prove via a standard argument a Caccioppoli inequality:

$$
\left(f_{B}|\nabla u|^{2} d w\right)^{1 / 2} \leq \frac{C_{1}}{r}\left(f_{2 B}\left|u-u_{2 B, w}\right|^{2} d w\right)^{1 / 2},
$$

where $C_{1}=C(n, \Lambda / \lambda)[w]_{A_{2}}^{1 / 2} \geq 1$. Fix $q$ such that

$$
\max \left\{\frac{2(n-1)}{n}, r_{w}, \frac{2 n r_{w}}{2+n r_{w}}\right\}<q<2 ;
$$

such a $q$ exists since $r_{w}<2$. Our choice of $q$ guarantees that $2<q_{w}^{*}$ and also that $2<$ $n q /(q-1)$. Then, by the weighted Poincaré inequality, Theorem 2.1,

$$
\frac{1}{r}\left(f_{2 B}\left|u-u_{2 B, w}\right|^{2} d w\right)^{1 / 2} \leq C_{2}\left(f_{2 B}|\nabla u|^{q} d w\right)^{1 / q},
$$

where $C_{2}=C(n)[w]_{A_{2}}^{\kappa} \geq 1$ and $\kappa=\frac{n q-1}{n q(q-1)}$. (By our choice of $q$ we can get this sharp estimate: see Remark 2.5. Since $q<2$ we could write $[w]_{A_{q}}$, but we use that $[w]_{A_{q}} \leq[w]_{A_{2}}$.) If we combine these inequalities, we get a reverse Hölder inequality:

$$
\left(f_{B}|\nabla u|^{2} d w\right)^{1 / 2} \leq C_{1} C_{2}\left(f_{2 B}|\nabla u|^{q} d w\right)^{1 / q} .
$$

We now apply Gehring's lemma in the setting of spaces of homogeneous type (see Björn and Björn [12, Theorem 3.22]) to get that there exists $p_{0}>2$ such that for every such $B$,

$$
\left(f_{B}|\nabla u|^{p_{0}} d w\right)^{1 / p_{0}} \leq C_{0}\left(f_{2 B}|\nabla u|^{2} d w\right)^{1 / 2} \text {. }
$$


Moreover, we can take the following values: $C_{0}=8 C_{1}^{2} C_{2}^{2}[w]_{A_{2}}^{31}$ and

$$
p_{0}=2+\frac{2-q}{2^{4 / q+1} C_{1}^{2} C_{2}^{2}[w]_{A_{2}}^{6 / q+17}} .
$$

In Section 11 below we will need these precise values. Here, it suffices to note that in inequality (8.3) we have $p_{0}>2$.

The Hodge projection. Define the Hodge projection operator by

$$
T=\nabla L_{w}^{-1 / 2}\left(\nabla\left(L_{w}^{*}\right)^{-1 / 2}\right)^{*},
$$

where the adjoint operators are defined with respect to the inner product in $L^{2}(w)$. As we noted in Section 2, the Riesz transform is bounded on $L^{2}(w)$; hence, the Hodge projection is also bounded. By duality, $\left(\nabla\left(L_{w}^{*}\right)^{-1 / 2}\right)^{*} \vec{f}=-L_{w}^{-1 / 2}\left(w^{-1} \operatorname{div}(w \vec{f})\right)$, and so

$$
T \vec{f}=-\nabla L_{w}^{-1 / 2} L_{w}^{-1 / 2}\left(w^{-1} \operatorname{div}(w \vec{f})=-\nabla L_{w}^{-1}\left(w^{-1} \operatorname{div}(w \vec{f})\right) .\right.
$$

Now fix $\vec{f} \in L^{2}\left(w, \mathbb{C}^{n}\right) \cap L^{p_{0}}\left(w, \mathbb{C}^{n}\right)$ such that $\operatorname{supp}(\vec{f}) \subset \mathbb{R}^{n} \backslash 4 B_{0}$. Let $u \in H^{1}(w)$ be a solution to the equation

$$
L_{w} u=w^{-1} \operatorname{div}(w \vec{f})
$$

by a standard Lax-Milgram argument because $A$ satisfies (2.7) (cf. [21, Theorem 2.2]), we know $u$ exists. Then

$$
T \vec{f}=-\nabla L_{w}^{-1} L_{w} u=-\nabla u,
$$

where equality is in the sense of distributions. In particular, since $f=0$ on $4 B_{0}, L_{w} u=0$ on $4 B_{0}$. Therefore, we can apply (8.3) to $u$ : on any ball $B$ such that $3 B \subset 4 B_{0}$,

$$
\left(f_{B}|T \vec{f}|^{p_{0}} d w\right)^{1 / p_{0}}=\left(f_{B}|\nabla u|^{p_{0}} d w\right)^{1 / p_{0}} \leq C_{0}\left(f_{2 B}|\nabla u|^{2} d w\right)^{1 / 2}=\left(f_{2 B}|T \vec{f}|^{2} d w\right)^{1 / 2} .
$$

As a consequence of this inequality, we have by [6, Theorem 3.14] (see also Section 5 of the same paper) that for all $q, 2 \leq q<p_{0}, T: L^{q}\left(w, \mathbb{C}^{n}\right) \rightarrow L^{q}\left(w, \mathbb{C}^{n}\right)$.

Boundedness of the Riesz transform. To show that the Riesz transform $\nabla L_{w}^{-1 / 2}$ is bounded, fix $q$ such that

$$
\max \left(p_{-}\left(L_{w}^{*}\right), r_{w}, p_{0}^{\prime}\right)=\max \left(p_{-}\left(L_{w}^{*}\right), r_{w}, p_{0}^{\prime}, \frac{n r_{w} p_{-}\left(L_{w}^{*}\right)}{n r_{w}+p_{-}\left(L_{w}^{*}\right)}\right)<q^{\prime}<2 .
$$

(The reason for including $p_{-}\left(L_{w}^{*}\right)$ will be made clear below.) By the above argument we have that $T^{*}$ is bounded on $L^{q^{\prime}}(w)$, where $T^{*} \vec{f}=-\nabla\left(L_{w}^{*}\right)^{-1}\left(w^{-1} \operatorname{div}(w \vec{f})\right)$. Furthermore, by Proposition 6.1, we have that

$$
\left\|\left(L_{w}^{*}\right)^{1 / 2} f\right\|_{L^{q^{\prime}}(w)} \leq C\|\nabla f\|_{L^{q^{\prime}}(w)} .
$$

Therefore,

$$
\begin{aligned}
\left\|\left(\nabla L_{w}^{-1 / 2}\right)^{*} \vec{f}\right\|_{L^{q^{\prime}}(w)} & =\left\|\left(L_{w}^{*}\right)^{-1 / 2}\left(w^{-1} \operatorname{div}(w \vec{f})\right)\right\|_{L^{q^{\prime}}(w)} \\
& =\left\|\left(L_{w}^{*}\right)^{1 / 2}\left(L_{w}^{*}\right)^{-1}\left(w^{-1} \operatorname{div}(w \vec{f})\right)\right\|_{L^{q^{\prime}}(w)} \\
& \lesssim\left\|\nabla\left(L_{w}^{*}\right)^{-1}\left(w^{-1} \operatorname{div}(w \vec{f})\right)\right\|_{L^{q^{\prime}}(w)} \\
& =\left\|T^{*} \vec{f}\right\|_{L^{q^{\prime}}(w)}
\end{aligned}
$$




$$
\lesssim\|\vec{f}\|_{L^{q^{\prime}}(w)} .
$$

Hence, by duality we have that $\nabla L_{w}^{-1 / 2}: L^{q}(w) \rightarrow L^{q}(w)$ for all $q$ such that

$$
2<q<\min \left(p_{+}\left(L_{w}\right), r_{w}^{\prime}, p_{0}\right)=q_{w} ;
$$

here we have used the fact that by duality, $p_{-}\left(L_{w}^{*}\right)^{\prime}=p_{+}\left(L_{w}\right)$.

Boundedness of the gradient of the semigroup. Finally, we show that if $2<q<q_{w}$, then $\sqrt{t} \nabla e^{-t L_{w}}: L^{q}(w) \rightarrow L^{q}(w)$. The desired estimate for $q_{+}\left(L_{w}\right)$ follows from this: by Proposition 7.1, part (4),

$$
q_{+}\left(L_{w}\right)=\sup \tilde{\mathcal{K}}\left(L_{w}\right) \geq q_{w}>2 .
$$

Fix such a $q$; then by the above estimate for the Riesz transform,

$$
\begin{array}{rl}
\left\|\sqrt{t} \nabla e^{-t L_{w}} f\right\|_{L^{q}(w)}=\| \nabla L_{w}^{-1 / 2}\left(t L_{w}\right)^{1 / 2} e^{-t L_{w}} & f \|_{L^{q}(w)} \\
& \lesssim\left\|\left(t L_{w}\right)^{1 / 2} e^{-t L_{w}} f\right\|_{L^{q}(w)}=\left\|\varphi_{t}\left(L_{w}\right) f\right\|_{L^{q}(w)},
\end{array}
$$

where $\varphi_{t}(z)=(t z)^{1 / 2} e^{-t z}$. For all $t>0$ this is a uniformly bounded holomorphic function in the right half plane. Therefore, since $2<q<p_{+}\left(L_{w}\right)$, by Proposition 4.3 we have that

$$
\left\|\sqrt{t} \nabla e^{-t L_{w}} f\right\|_{L^{q}(w)} \lesssim\left\|\varphi_{t}\right\|_{\infty}\|f\|_{L^{q}(w)} \lesssim\|f\|_{L^{q}(w)}
$$

and the bound is independent of $t$. This completes the proof that $q_{+}\left(L_{w}\right)>2$.

\section{RiESZ TRANSFORM ESTIMATES}

In this section we prove $L^{p}(w)$ norm inequalities for the Riesz transform $\nabla L_{w}^{-1 / 2}$. We have already proved such inequalities for a small range of values $q>2$ in Section 8 . Here we prove the following result.

Proposition 9.1. Let $q_{-}\left(L_{w}\right)<p<q_{+}\left(L_{w}\right)$. Then there exists a constant $C$ such that

$$
\left\|\nabla L_{w}^{-1 / 2} f\right\|_{L^{p}(w)} \leq C\|f\|_{L^{p}(w)} .
$$

Furthermore, if $v \in A_{p / q_{-}\left(L_{w}\right)}(w) \cap R H_{\left(q_{+}\left(L_{w}\right) / p\right)^{\prime}}(w)$, then

$$
\left\|\nabla L_{w}^{-1 / 2} f\right\|_{L^{p}(v d w)} \leq C\|f\|_{L^{p}(v d w)} .
$$

To prove Proposition 9.1 we would like to follow the same outline as the proof of Proposition 4.3. The first step-i.e., proving (9.2) holds when $q_{-}\left(L_{w}\right)<p<2$ - does work with the appropriate changes. However, the second step (i.e., the proof that (9.3) holds) runs into difficulties since $\nabla L_{w}^{-1 / 2}$ and the auxiliary operators $\mathcal{A}_{r}$ do not commute. One approach to overcoming this obstacle would be to adapt the proof in [5] (see also [2]). In this case we would need to use an $L^{p_{0}}(w)$-Poincaré inequality which may not hold unless we assume $w \in A_{p_{0}}$. This would yield estimates in the range $\max \left\{r_{w}, q_{-}\left(L_{w}\right)\right\}<p<q_{+}\left(L_{w}\right)$, analogous to those in Proposition 6.1.

There is, however, an alternative approach. In [8] the authors considered Riesz transforms associated with the Laplace-Beltrami operator of a complete, non-compact Riemannian manifold. Their proof avoids Poincaré inequalities for $p$ close to 1 as these may not hold. Instead, they use a duality argument based on ideas in [11]; this requires that they first prove that the Riesz transform is bounded for $p>2$ in the appropriate range of values. This reverses the order used in the proof of Proposition 4.3. 
Proof of Proposition 9.1. For brevity, let $q_{-}=q_{-}\left(L_{w}\right)$ and $q_{+}=q_{+}\left(L_{w}\right)$. To implement the approach sketched above, we divide the proof in two steps. First we will prove that (9.2) holds when $2<p<q_{+}$. We do so using Theorem 2.35 and some ideas from [2, 5]. We note that since the Riesz transform and $\mathcal{A}_{r}$ do not commute, we will use an $L^{2}(w)$-Poincaré inequality. This holds since $w \in A_{2}$ : the problem with using the Poincaré inequality only occurs with exponents less than 2. The second step is to prove that (9.3) holds by adapting the proof in [8]. Here we will use duality and a result from [6] that is based on good- $\lambda$ inequalities. Inequality (9.2) then holds when $q_{-}<p<2$ by taking $v \equiv 1$.

To apply Theorem 2.35, fix $2<p<q_{+}$and let $T=\nabla L_{w}^{-1 / 2}, S=I$ and $\mathcal{D}=L_{c}^{\infty}$. Let $p_{0}=2$ and fix $q_{0}$ such that $2<p<q_{0}<q_{+}$. As before we take $\mathcal{A}_{r}=I-\left(I-e^{-r^{2} L_{w}}\right)^{m}$, where $m$ will be chosen below. We first show that (2.36) holds. Let $f \in L_{c}^{\infty}$ and decompose it as in (4.11); then we have

$$
\left(f_{B}\left|\nabla L_{w}^{-1 / 2}\left(I-e^{-r^{2} L_{w}}\right)^{m} f\right|^{2} d w\right)^{\frac{1}{2}} \leq \sum_{j \geq 1}\left(f_{B}\left|\nabla L_{w}^{-1 / 2}\left(I-e^{-r^{2} L_{w}}\right)^{m} f_{j}\right|^{2} d w\right)^{\frac{1}{2}} .
$$

To estimate the first term, note that $\nabla L_{w}^{-1 / 2}$ and $e^{-r^{2} L_{w}}$ are bounded on $L^{2}(w)$ by Theorems 2.15 and 2.18. Hence,

$$
\left(f_{B}\left|\nabla L_{w}^{-1 / 2}\left(I-e^{-r^{2} L_{w}}\right)^{m} f_{1}\right|^{2} d w\right)^{\frac{1}{2}} \lesssim\left(f_{4 B}|f|^{2} d w\right)^{\frac{1}{2}}
$$

Fix $j \geq 2$; to get the desired $L^{2}$ estimates we will use the $L^{2}$ bounds for the gradient of the square function.. If $h \in L^{2}(w)$, by $(2.20)$

$$
\nabla L_{w}^{-1 / 2}\left(I-e^{-r^{2} L_{w}}\right)^{m} h=\frac{1}{\sqrt{\pi}} \int_{0}^{\infty} \sqrt{t} \nabla \varphi\left(L_{w}, t\right) h \frac{d t}{t}
$$

where $\varphi(z, t)=e^{-t z}\left(1-e^{-r^{2} z}\right)^{m} \in \mathcal{H}_{0}^{\infty}\left(\Sigma_{\mu}\right)$. We can therefore use the integral representation $(2.10)$ for $\varphi(\cdot, t)$. The function $\eta(\cdot, t)$ in this representation satisfies

$$
|\eta(z, t)| \lesssim \frac{r^{2 m}}{(|z|+t)^{m+1}}, \quad z \in \Gamma, t>0 .
$$

By Theorem 2.15, $\sqrt{z} \nabla e^{-z L_{w}} \in \mathcal{O}\left(L^{2}(w) \rightarrow L^{2}(w)\right)$; hence,

$$
\begin{aligned}
& \left(f_{B}\left|\int_{\Gamma} \eta(z) \sqrt{t} \nabla e^{-z L_{w}} f_{j} d z\right|^{2} d w\right)^{\frac{1}{2}} \\
& \quad \leq \int_{\Gamma}\left(f_{B}\left|\sqrt{z} \nabla e^{-z L_{w}} f_{j}\right|^{2} d w\right)^{\frac{1}{2}} \frac{\sqrt{t}}{\sqrt{|z|}}|\eta(z)||d z| \\
& \quad \lesssim 2^{j \theta_{1}} \int_{\Gamma} \Upsilon\left(\frac{2^{j} r}{\sqrt{|z|}}\right)^{\theta_{2}} e^{-\frac{\alpha 4^{j} r^{2}}{|z|}} \frac{\sqrt{t}}{\sqrt{|z|}}|\eta(z)||d z|\left(f_{C_{j}(B)}|f|^{2} d w\right)^{\frac{1}{2}} \\
& \quad \lesssim 2^{j \theta_{1}} \int_{0}^{\infty} \Upsilon\left(\frac{2^{j} r}{\sqrt{s}}\right)^{\theta_{2}} e^{-\frac{\alpha 4^{j} r^{2}}{s}} \frac{\sqrt{t}}{\sqrt{s}} \frac{r^{2 m}}{(s+t)^{m+1}} d s\left(f_{C_{j}(B)}|f|^{2} d w\right)^{\frac{1}{2}} .
\end{aligned}
$$


When $2 m>\theta_{2}$,

$$
\int_{0}^{\infty} \int_{0}^{\infty} \Upsilon\left(\frac{2^{j} r}{\sqrt{s}}\right)^{\theta_{2}} e^{-\frac{\alpha 4^{j} r^{2}}{s}} \frac{\sqrt{t}}{\sqrt{s}} \frac{r^{2 m}}{(s+t)^{m+1}} d s \frac{d t}{t}=C 4^{-j m} .
$$

If we insert this into the representation (2.10) we get

$$
\begin{aligned}
\left(f_{B}\left|\nabla e^{-t L_{w}}\left(I-e^{-r^{2} L_{w}}\right)^{m} f_{j}\right|^{2} d w\right)^{\frac{1}{2}} & \lesssim \int_{0}^{\infty}\left(f_{B}\left|\sqrt{t} \nabla \varphi\left(L_{w}, t\right) f_{j}\right|^{2} d w\right)^{\frac{1}{2}} \frac{d t}{t} \\
& \lesssim 2^{j\left(\theta_{1}-2 m\right)}\left(f_{C_{j}(B)}|f|^{2} d w\right)^{\frac{1}{2}}
\end{aligned}
$$

If we now combine (9.4) and (9.8) we get (2.36) with $g(j)=C_{m} 2^{j\left(\theta_{1}-2 m\right)}$; if we also fix $2 m>\theta_{1}$, we get that $\sum g(j)<\infty$.

We now show that (2.37) holds. As we remarked above, the Riesz transform does not commute with $\mathcal{A}_{r}$. To overcome this obstacle, we will prove an off-diagonal estimate for the gradient of the semigroup (using the $L^{2}(w)$-Poincaré inequality), and then use an approximation argument to get the desired estimate for the Riesz transform.

More precisely, we claim that for every $f \in H^{1}(w)$ and $1 \leq k \leq m$,

$$
\left(f_{B}\left|\nabla e^{-k r^{2} L_{w}} f\right|^{q_{0}} d w\right)^{\frac{1}{q_{0}}} \leq \sum_{j \geq 1} g(j)\left(f_{2^{j+1} B}|\nabla f|^{2} d w\right)^{\frac{1}{2}}
$$

where $g(j)=C_{m} 2^{j} \sum_{l \geq j} 2^{l \theta} e^{-\alpha 4^{l}}$. Assume for the moment that (9.9) holds. Then for every $\epsilon>0$ we can apply this estimate to $S_{\varepsilon} f$ (defined by (2.21)) since $S_{\varepsilon} f \in H^{1}(w)$. Moreover, we have that $\mathcal{A}_{r}$ and $S_{\varepsilon}$ commute, and so if we expand $\mathcal{A}_{r}=I-\left(I-e^{-r^{2} L}\right)^{m}$ and apply (9.9), we get

$$
\left(f_{B}\left|\nabla S_{\varepsilon} \mathcal{A}_{r} f\right|^{q_{0}} d w\right)^{\frac{1}{q_{0}}} \leq C_{m} \sum_{j \geq 1} g(j)\left(f_{2^{j+1} B}\left|\nabla S_{\varepsilon} f\right|^{2} d w\right)^{\frac{1}{2}} .
$$

If we let $\varepsilon$ go to 0 , we obtain (2.37). (The justification of this uses the observations made in Section 2 after (2.21) and is left to the reader.) Moreover, we have that $\sum_{j \geq 1} g(j)<\infty$, and so by Theorem 2.35 with $v \equiv 1$ (which trivially satisfies $v \in A_{p / 2}(w) \cap R H_{\left(q_{0} / p\right)^{\prime}}(w)$ ) we have that (9.2) holds for $f \in L_{c}^{\infty}$ and for every $2<p<q_{+}$.

To complete this step we need to prove (9.9). Fix $1 \leq k \leq m$ and $f \in H^{1}(w)$. Let $h=f-f_{4 B, w}$, where $f_{4 B, w}=f_{4 B} f d w$. Then by the conservation property (see [19], or the proof in [2, Section 2.5]), $e^{-t L_{w}} 1=1$ for all $t>0$, and so

$$
\nabla e^{-k r^{2} L_{w}} f=\nabla e^{-k r^{2} L_{w}}\left(f-f_{4 B, w}\right)=\nabla e^{-k r^{2} L_{w}} h=\sum_{j \geq 1} \nabla e^{-k r^{2} L_{w}} h_{j},
$$

where $h_{j}=h \chi_{C_{j}(B)}$. Hence,

$$
\left(f_{B}\left|\nabla e^{-k r^{2} L_{w}} f\right|^{q_{0}} d w\right)^{\frac{1}{q_{0}}} \leq \sum_{j \geq 1}\left(f_{B}\left|\nabla e^{-k r^{2} L_{w}} h_{j}\right|^{q_{0}} d w\right)^{\frac{1}{q_{0}}} .
$$

Since $2<q_{0}<q_{+}$, by Proposition 7.1, $\sqrt{t} \nabla e^{-t L_{w}} \in \mathcal{O}\left(L^{2}(w) \rightarrow L^{q_{0}}(w)\right)$. If we apply this and the $L^{2}(w)$-Poincaré inequality (see Remark 2.6 with $p=q=2$ ), then for each $j \geq 1$ we 
get

$$
\begin{aligned}
\left(f_{B} \mid\right. & \left.\left.\nabla e^{-k r^{2} L_{w}} h_{j}\right|^{q_{0}} d w\right)^{\frac{1}{q_{0}}} \\
& \lesssim \frac{2^{j\left(\theta_{1}+\theta_{2}\right)} e^{-\alpha 4^{j}}}{r}\left(f_{C_{j}(B)}\left|h_{j}\right|^{2} d w\right)^{\frac{1}{2}} \\
& \leq \frac{2^{j\left(\theta_{1}+\theta_{2}\right)} e^{-\alpha 4^{j}}}{r}\left(f_{2^{j+1} B}\left|f-f_{4 B, w}\right|^{2} d w\right)^{\frac{1}{2}} \\
& \leq \frac{2^{j\left(\theta_{1}+\theta_{2}\right)} e^{-\alpha 4^{j}}}{r}\left(\left(f_{2^{j+1} B}\left|f-f_{2^{j+1} B, w}\right|^{2} d w\right)^{\frac{1}{2}}+\sum_{l=2}^{j}\left|f_{2^{l} B, w}-f_{2^{l+1} B, w}\right|\right) \\
& \lesssim \frac{2^{j\left(\theta_{1}+\theta_{2}\right)} e^{-\alpha 4^{j}}}{r} \sum_{l=1}^{j}\left(f_{2^{l+1} B}\left|f-f_{2^{l+1} B, w}\right|^{2} d w\right)^{\frac{1}{2}} \\
& \lesssim 2^{j\left(\theta_{1}+\theta_{2}\right)} e^{-\alpha 4^{j}} \sum_{l=1}^{j} 2^{l}\left(f_{2^{l+1} B}|\nabla f|^{2} d w\right)^{\frac{1}{2}} \cdot
\end{aligned}
$$

If we combine these two estimates and exchange the order of summation we get (9.9) with $\theta=\theta_{1}+\theta_{2}$. This completes the proof that (9.2) holds when $2<p<q_{+}$.

For the second step of our proof we show that (9.3) holds for all $p, q_{-}<p<q_{+}$and $v \in A_{p / q_{-}}(w) \cap R H_{\left(q_{+} / p\right)^{\prime}}(w)$. Fix such a $p$ and $v$; then by the openness properties of $A_{q}$ and $R H_{s}$ weights, there exist $p_{0}, q_{0}$ such that

$$
q_{-}<p_{0}<\min \{p, 2\} \leq \max \{p, 2\}<q_{0}<q_{+} \quad \text { and } \quad v \in A_{p / p_{0}}(w) \cap R H_{\left(q_{0} / p\right)^{\prime}}(w) .
$$

By the duality properties of weights [6, Lemma 4.4],

$$
u=v^{1-p^{\prime}} \in A_{p^{\prime} / q_{0}^{\prime}}(w) \cap R H_{\left(p_{0}^{\prime} / p^{\prime}\right)^{\prime}}(w) .
$$

Let $T=\nabla L_{w}^{-1 / 2}$; then $T$ is bounded from $L^{p}\left(\mathbb{R}^{n}, v d w\right)$ to $L^{p}\left(\mathbb{R}^{n} ; \mathbb{C}^{n}, v d w\right)$ if and only if $T^{*}$ is bounded from $L^{p^{\prime}}\left(\mathbb{R}^{n} ; \mathbb{C}^{n}, u d w\right)$ to $L^{p^{\prime}}\left(\mathbb{R}^{n} ; u d w\right)$. (Note that $T$ takes scalar valued functions to vector functions valued and $T^{*}$ the opposite.)

Therefore, it will suffice to prove the boundedness of $T^{*}$. We will do so using a particular case of [6, Theorem 3.1]. This result is stated there in the Euclidean setting but it extends to spaces of homogeneous type. Here we give the weighted version we need: see [6, Section $5]$.

Theorem 9.10. Fix $1<q<\infty, a \geq 1$ and $u \in R H_{s^{\prime}}(w), 1<s<\infty$. Then there exists $C>1$ with the following property: suppose $F \in L^{1}(w)$ and $G$ are non-negative measurable functions such that for any ball $B$ there exist non-negative functions $G_{B}$ and $H_{B}$ with $F(x) \leq G_{B}(x)+H_{B}(x)$ for a.e. $x \in B$ and, for all $x \in B$,

$$
\left(f_{B} H_{B}^{q} d w\right)^{\frac{1}{q}} \leq a M_{w} F(x), \quad \quad f_{B} G_{B} d w \leq G(x)
$$

where $M_{w}$ is the Hardy-Littlewood maximal function with respect to dw. Then for $1<t<$ $q / s$,

$$
\left\|M_{w} F\right\|_{L^{t}(u d w)} \leq C\|G\|_{L^{t}(u d w)}
$$


To apply Theorem 9.10, fix $\vec{f} \in L_{c}^{\infty}\left(\mathbb{R}^{n} ; \mathbb{C}^{n}\right)$, and let $h=T^{*} \vec{f}$ and $F=|h|^{q_{0}^{\prime}}$. Then $F \in L^{1}(w)$ : by the argument above, since $2<q_{0}<q_{+}, T$ is bounded from $L^{q_{0}}\left(\mathbb{R}^{n}, w\right)$ to $L^{q_{0}}\left(\mathbb{R}^{n} ; \mathbb{C}^{n}, w\right)$, thus $T^{*}$ is bounded from $L^{q_{0}^{\prime}}\left(\mathbb{R}^{n} ; \mathbb{C}^{n}, w\right)$ to $L^{q_{0}^{\prime}}\left(\mathbb{R}^{n}, w\right)$.

Now let $\mathcal{A}_{r}=I-\left(I-e^{-r^{2} L_{w}}\right)^{m}$, where $m>0$ will be fixed below. Given a ball $B$ with radius $r$, we define

$$
F \leq 2^{q_{0}^{\prime}-1}\left|\left(I-\mathcal{A}_{r}\right)^{*} h\right|^{q_{0}^{\prime}}+2^{q_{0}^{\prime}-1}\left|\mathcal{A}_{r}^{*} h\right|^{q_{0}^{\prime}} \equiv G_{B}+H_{B}
$$

where, as before, the adjoint is with respect to $L^{2}(w)$. To complete the proof, suppose for the moment that we could prove (9.11) with $q=p_{0}^{\prime} / q_{0}^{\prime}$ and $G=M_{w}\left(|\vec{f}|_{0}^{\prime}\right)$. Since $u \in R H_{\left(p_{0}^{\prime} / p^{\prime}\right)^{\prime}}(w)$, by the openness property of reverse Hölder weights, $u \in R H_{s^{\prime}}(w)$ for some $s<p_{0}^{\prime} / p^{\prime}$. Then if we let $t=p^{\prime} / q_{0}^{\prime}=\left(p_{0}^{\prime} / q_{0}^{\prime}\right) /\left(p_{0}^{\prime} / p^{\prime}\right)<q / s$, we have $u \in A_{t}(w)$, and so $M_{w}$ is bounded on $L^{t}(u d w)$. Therefore, by $(9.12)$,

$$
\left\|T^{*} \vec{f}\right\|_{L^{p^{\prime}(u d w)}}^{q^{\prime}} \leq\left\|M_{w} F\right\|_{L^{t}(u d w)} \leq C\|G\|_{L^{t}(u d w)}=C\left\|M_{w}\left(|\vec{f}|^{q_{0}^{\prime}}\right)\right\|_{L^{t}(u d w)} \lesssim\|\vec{f}\|_{L^{p^{\prime}(u d w)}}^{q^{\prime}} .
$$

To complete the proof we need to show that (9.11) holds. We first estimate $H_{B}$. By duality there exists $g \in L^{p_{0}}(B, d w / w(B))$ with norm 1 such that for all $x \in B$,

$$
\begin{aligned}
\left(f_{B} H_{B}^{q} d w\right)^{\frac{1}{q q_{0}^{\prime}}} & \lesssim w(B)^{-1} \int_{\mathbb{R}^{n}}|h|\left|\mathcal{A}_{r} g\right| d w \\
& \lesssim \sum_{j=1}^{\infty} 2^{j D}\left(f_{C_{j}(B)}|h|^{q_{0}^{\prime}} d w\right)^{\frac{1}{q_{0}^{\prime}}}\left(f_{C_{j}(B)}\left|\mathcal{A}_{r} g\right|^{q_{0}} d w\right)^{\frac{1}{q_{0}}} \\
& \lesssim M_{w} F(x)^{\frac{1}{q_{0}^{\prime}}} \sum_{j=1}^{\infty} 2^{j\left(D+\theta_{1}+\theta_{2}\right)} e^{-\alpha 4^{j}}\left(f_{B}|g|^{p_{0}} d w\right)^{\frac{1}{p_{0}}} \\
& \lesssim M_{w} F(x)^{\frac{1}{q_{0}^{\prime}}}
\end{aligned}
$$

where in the second to last inequality we used the fact that by our choice of $p_{0}, q_{0}, e^{-t L_{w}} \in$ $\mathcal{O}\left(L^{p_{0}}(w) \rightarrow L^{q_{0}}(w)\right)$, and so $\mathcal{A}_{r}$ is as well.

We now estimate $G_{B}$. Again by duality there exists $g \in L^{q_{0}}(B, d w / w(B))$ with norm 1 such that for all $x \in B$,

$$
\begin{aligned}
\left(f_{B} G_{B} d w\right)^{\frac{1}{q_{0}^{\prime}}} & \lesssim w(B)^{-1} \int_{\mathbb{R}^{n}}|\vec{f}|\left|T\left(I-\mathcal{A}_{r}\right) g\right| d w \\
& \lesssim \sum_{j=1}^{\infty} 2^{j D}\left(f_{C_{j}(B)}|\vec{f}|^{q_{0}^{\prime}} d w\right)^{\frac{1}{q_{0}^{\prime}}}\left(f_{C_{j}(B)}\left|T\left(I-\mathcal{A}_{r}\right) g\right|^{q_{0}} d w\right)^{\frac{1}{q_{0}}} \\
& \leq M_{w}\left(|\vec{f}|^{q_{0}^{\prime}}\right)(x)^{\frac{1}{q_{0}^{\prime}}} \sum_{j=1}^{\infty} 2^{j D}\left(f_{C_{j}(B)}\left|T\left(I-\mathcal{A}_{r}\right) g\right|^{q_{0}} d \mu\right)^{\frac{1}{q_{0}}}
\end{aligned}
$$

To estimate each term in the sum we argue as in the first half of the proof. When $j=1$, $\nabla L_{w}^{-1 / 2}$ and $e^{-r^{2} L_{w}}$ are bounded on $L^{q_{0}}(w)$ by the first part of the proof and Theorem 2.15. Hence,

$$
\left(f_{4 B}\left|\nabla L_{w}^{-1 / 2}\left(I-e^{-r^{2} L_{w}}\right)^{m} g\right|^{q_{0}} d w\right)^{\frac{1}{q_{0}}} \lesssim\left(f_{B}|g|^{q_{0}} d w\right)^{\frac{1}{q_{0}}}=1
$$


For $j \geq 2$ we use the integral representation (9.5). If we estimate as in (9.6), with the roles of $B$ and $C_{j}(B)$ switched and using the fact that $\sqrt{z} \nabla e^{-z L_{w}} \in \mathcal{O}\left(L^{q_{0}}(w) \rightarrow L^{q_{0}}(w)\right)$ since $2<q_{0}<q_{+}$, we see that

$$
\begin{aligned}
& \left(f_{C_{j}(B)}\left|\int_{\Gamma} \eta(z) \sqrt{t} \nabla e^{-z L_{w}} g d z\right|^{q_{0}} d w\right)^{\frac{1}{q_{0}}} \\
& \quad \leq \int_{\Gamma}\left(f_{C_{j}(B)}\left|\sqrt{z} \nabla e^{-z L_{w}} g\right|^{q_{0}} d w\right)^{\frac{1}{q_{0}}} \frac{\sqrt{t}}{\sqrt{|z|}}|\eta(z)||d z| \\
& \quad \lesssim 2^{j \theta_{1}} \int_{\Gamma} \Upsilon\left(\frac{2^{j} r}{\sqrt{|z|}}\right)^{\theta_{2}} e^{-\frac{\alpha 4^{j} r^{2}}{|z|}} \frac{\sqrt{t}}{\sqrt{|z|}}|\eta(z)||d z|\left(f_{B}|g|^{q_{0}} d w\right)^{\frac{1}{2}} \\
& \lesssim 2^{j \theta_{1}} \int_{0}^{\infty} \Upsilon\left(\frac{2^{j} r}{\sqrt{s}}\right)^{\theta_{2}} e^{-\frac{\alpha 4^{j} r^{2}}{s}} \frac{\sqrt{t}}{\sqrt{s}} \frac{r^{2 m}}{(s+t)^{m+1}} d s .
\end{aligned}
$$

If we take $2 m>\theta_{2}$, we can combine this with (9.7). We can then insert this estimate into the representation (2.10) to get that for every $j \geq 2$,

$$
\begin{aligned}
\left(f_{C_{j}(B)}\left|\nabla e^{-t L_{w}}\left(I-e^{-r^{2} L_{w}}\right)^{m} g\right|^{q_{0}} d w\right)^{\frac{1}{q_{0}}} & \\
& \lesssim \int_{0}^{\infty}\left(f_{C_{j}(B)}\left|\sqrt{t} \nabla \varphi\left(L_{w}, t\right) g\right|^{q_{0}} d w\right)^{\frac{1}{q_{0}}} \frac{d t}{t} \lesssim 2^{j\left(\theta_{1}-2 m\right)} .
\end{aligned}
$$

Taken together, (9.13), (9.14) and (9.15) yield

$$
\left(f_{B} G_{B} d w\right)^{\frac{1}{q_{0}^{\prime}}} \lesssim M_{w}\left(|\vec{f}|^{q_{0}^{\prime}}\right)(x)^{\frac{1}{q_{0}^{\prime}}} \sum_{j=1}^{\infty} 2^{j\left(D+\theta_{1}-2 m\right)} \lesssim M_{w}\left(|\vec{f}|^{q_{0}^{\prime}}\right)(x)^{\frac{1}{q_{0}^{\prime}}}=G(x)^{\frac{1}{q_{0}^{\prime}}}
$$

provided we take $m$ large enough so that $D+\theta_{1}-2 m<0$. This completes the estimate of $H_{B}$ and $G_{B}$ and so completes our proof.

\section{SquARE FunCtion ESTimates FOR the GRADiEnt of THE SEMigroup}

In this section we prove $L^{p}(w)$ estimates for the vertical square function

$$
G_{L_{w}} f(x)=\left(\int_{0}^{\infty}\left|t^{1 / 2} \nabla e^{-t L_{w}} f(x)\right|^{2} \frac{d t}{t}\right)^{1 / 2} .
$$

Proposition 10.1. Let $q_{-}\left(L_{w}\right)<p<q_{+}\left(L_{w}\right)$. Then

$$
\left\|G_{L_{w}} f\right\|_{L^{p}(w)} \lesssim\|f\|_{L^{p}(w)} .
$$

Furthermore, if $v \in A_{p / q_{-}\left(L_{w}\right)}(w) \cap R H_{\left(q_{+}\left(L_{w}\right) / p\right)^{\prime}}(w)$, then

$$
\left\|G_{L_{w}} f\right\|_{L^{p}(v d w)} \lesssim\|f\|_{L^{p}(v d w)} .
$$

We can also prove a reverse inequality for $G_{L_{w}}$. To do so we need to introduce an auxiliary operator. Define the weighted Laplacian by $\Delta_{w}=-w^{-1} \operatorname{div} w \nabla$ : i.e., $\Delta_{w}$ is the operator $L_{w}$ if we take the matrix $A$ to be $w I$, where $I$ is the identity matrix. 
Proposition 10.4. Let $q_{+}\left(\Delta_{w}\right)^{\prime}<p<\infty$. Then

$$
\|f\|_{L^{p}(w)} \lesssim\left\|G_{L_{w}} f\right\|_{L^{p}(w)} .
$$

Furthermore, if $v \in A_{p / q_{+}\left(\Delta_{w}\right)^{\prime}}(w)$, then

$$
\|f\|_{L^{p}(v d w)} \lesssim\left\|G_{L_{w}} f\right\|_{L^{p}(v d w)} .
$$

Proof of Proposition 10.1. The proof could be done in a way similar to those for the square function $g_{L_{w}}$ in Section 5. However, we will give a shorter proof that uses the Riesz transform estimates from Section 9.

Let $q_{-}=q_{-}\left(L_{w}\right)$ and $q_{+}=q_{+}\left(L_{w}\right)$. Fix $p$,

$$
q_{-}=p_{-}\left(L_{w}\right)<p<q_{+} \leq p_{+}\left(L_{w}\right)
$$

and $v \in A_{p / q_{-}}(w) \cap R H_{\left(q_{+} / p\right)^{\prime}}(w)$. Then by Proposition 9.1, the Riesz transform is bounded on $L^{p}(v d w)$, and so by Lemma 5.4 it has a bounded extension to $L_{\mathbb{H}}^{p}(v d w)$ : i.e., if $g(x, t) \in$ $L_{\mathbb{H}}^{p}(v d w)$, then $\left\|\nabla L_{w}^{-1 / 2} g\right\|_{L_{\mathbb{H}}^{p}(v d w)} \lesssim\|g\|_{L_{\mathbb{H}}^{p}(v w)}$, where the extension of $\nabla L_{w}^{-1 / 2}$ to $\mathbb{H}$-valued functions is defined for $x \in \mathbb{R}^{n}$ and $t>0$ by $\left(\nabla L_{w}^{-1 / 2} g\right)(x, t)=\nabla L_{w}^{-1 / 2}(g(\cdot, t))(x)$.

Define $g_{f}(x, t)=\left(t L_{w}\right)^{1 / 2} e^{-t L_{w}} f(x)$ and $G_{f}(x, t)=t^{1 / 2} \nabla e^{-t L_{w}} f(x)$; then we clearly have $\left\|g_{L_{w}} f\right\|_{L^{p}(v d w)}=\left\|g_{f}\right\|_{L_{\mathbb{H}}^{p}(v d w)}$ and $\left\|G_{L_{w}} f\right\|_{L^{p}(v d w)}=\left\|G_{f}\right\|_{L_{\mathbb{H}}^{p}(v d w)}$. Furthermore, $G_{f}(x, t)=$ $\nabla L_{w}^{-1 / 2}\left(g_{f}(\cdot, t)\right)(x)=\left(\nabla L_{w}^{-1 / 2} g_{f}\right)(x, t)$. Hence,

$$
\begin{aligned}
\left\|G_{L_{w}} f\right\|_{L^{p}(v d w)}=\left\|G_{f}\right\|_{L_{\mathbb{H}}^{p}(v d w)}=\| \nabla L_{w}^{-1 / 2} & g_{f} \|_{L_{\mathbb{H}}^{p}(v d w)} \\
& \lesssim\left\|g_{f}\right\|_{L_{\mathbb{H}}^{p}(v d w)}=\left\|g_{L_{w}} f\right\|_{L^{p}(v d w)} \lesssim\|f\|_{L^{p}(v d w)} .
\end{aligned}
$$

To prove the last inequality we used Proposition 5.1; we also used the fact that $q_{-}=$ $p_{-}\left(L_{w}\right)<p<q_{+} \leq p_{+}\left(L_{w}\right)$ and $v \in A_{p / q_{-}}(w) \cap R H_{\left(q_{+} / p\right)^{\prime}}(w)$, which together imply that $v \in A_{p / p_{-}\left(L_{w}\right)}(w) \cap R H_{\left(p_{+}\left(L_{w}\right) / p\right)^{\prime}}(w)$. This proves (10.3). To prove inequality (10.2), we take $v \equiv 1$.

To prove Proposition 10.4 we need the following identity relating $G_{L_{w}}$ and $\Delta_{w}$. It is a straightforward extension of a similar unweighted result given in [2, Section 7.1]. For completeness we include the proof.

Lemma 10.7. If $f, g \in L_{c}^{\infty}(w)$ then

$$
\left|\int_{\mathbb{R}^{n}} f(x) \overline{g(x)} d w\right| \leq(\Lambda+1) \int_{\mathbb{R}^{n}} G_{L_{w}} f(x) \overline{G_{\Delta_{w}} g(x)} d w .
$$

Proof. By the definition and properties of the operators $L_{w}$ and $\Delta_{w}$ we have that

$$
\begin{aligned}
\int_{\mathbb{R}^{n}} f(x) \overline{g(x)} d w & \\
& =\lim _{\varepsilon \downarrow 0} \int_{\mathbb{R}^{n}} e^{-\varepsilon L_{w}} f(x) \overline{e^{-\varepsilon \Delta_{w}} g(x)} d w-\lim _{R \uparrow \infty} \int_{\mathbb{R}^{n}} e^{-R L_{w}} f(x) \overline{e^{-R \Delta_{w}} g(x)} d w \\
& =-\int_{0}^{\infty} \frac{d}{d t} \int_{\mathbb{R}^{n}} e^{-t L_{w}} f(x) \overline{e^{-t \Delta_{w}} g(x)} d w d t \\
& =\int_{0}^{\infty} \int_{\mathbb{R}^{n}}\left(L_{w} e^{-t L_{w}} f(x) \overline{e^{-t \Delta_{w}} g(x)}+e^{-t L_{w}} f(x) \overline{\Delta_{w} e^{-t \Delta_{w}} g(x)}\right) d w d t
\end{aligned}
$$




$$
=\int_{0}^{\infty} \int_{\mathbb{R}^{n}}\left(A(x) w(x)^{-1}+I\right)\left(\nabla e^{-t L_{w}} f(x) \overline{\nabla e^{-t \Delta_{w}} g(x)}\right) d w d t .
$$

Since $\left\|A w^{-1}\right\|_{\infty} \leq \Lambda$, if we apply Hölder's inequality in the $t$ variable we get the desired result.

Proof of Proposition 10.4. As a consequence of the Gaussian estimate for weighted operators with real symmetric coefficients that were proved in $[16,18]$, we have that $\Delta_{w} \in \mathcal{O}\left(L^{1}(w) \rightarrow\right.$ $\left.L^{\infty}(w)\right)$. In particular, $q_{-}\left(\Delta_{w}\right)=p_{-}\left(L_{\Delta_{w}}\right)=1$. Further, by the results in Section 8 we have that $q_{+}\left(\Delta_{w}\right)>2$.

Therefore, by Proposition 10.1, if $1<p^{\prime}<q_{+}\left(\Delta_{w}\right)$, and

$$
u \in A_{p^{\prime}}(w) \cap R H_{\left(q_{+}\left(\Delta_{w}\right) / p^{\prime}\right)^{\prime}}(w),
$$

then

$$
\left\|G_{\Delta_{w}} f\right\|_{L^{p^{\prime}(u d w)}} \lesssim\|f\|_{L^{p^{\prime}(u d w)}} \cdot
$$

We want to apply inequality (10.9) with $u=v^{1-p^{\prime}}$. By [6, Lemma 4.4], the condition (10.8) is equivalent to $v \in A_{p / q_{+}(w)^{\prime}}(w)$.

Now fix $f, g \in L_{c}^{\infty}$, and a weight $v \in A_{p / q_{+}(w)^{\prime}}(w)$. Then by Lemma 10.7, for $q_{+}\left(\Delta_{w}\right)^{\prime}<$ $p<\infty$,

$$
\begin{aligned}
\left|\int_{\mathbb{R}^{n}} f(x) g(x) d w\right| & \leq(\Lambda+1) \int_{\mathbb{R}^{n}} G_{L_{w}} f(x) G_{\Delta_{w}} g(x) d w \\
& =(\Lambda+1) \int_{\mathbb{R}^{n}} G_{L_{w}} f(x) G_{\Delta_{w}} g(x) v^{1 / p} v^{-1 / p} d w \\
& \leq(\Lambda+1)\left\|G_{L_{w}} f\right\|_{L^{p}(v d w)}\left\|G_{\Delta_{w}} g\right\|_{L^{p^{\prime}\left(v^{1-p^{\prime}} d w\right)}} \\
& \lesssim\left\|G_{L_{w}} f\right\|_{L^{p}(v d w)}\|g\|_{L^{p^{\prime}\left(v^{1-p^{\prime}} d w\right)}} ;
\end{aligned}
$$

the last inequality follows from (10.9). If we take $g=\operatorname{sign}(f)|f|^{p-1} v$, we get

$$
\|f\|_{L^{p}(v d w)}^{p} \lesssim\left\|G_{L_{w}} f\right\|_{L^{p}(v w)}\left\||f|^{p-1} v\right\|_{L^{p^{\prime}\left(v^{1-p^{\prime}} d w\right)}}=\left\|G_{L_{w}} f\right\|_{L^{p}(v d w)}\|f\|_{L^{p}(v d w)}^{p / p^{\prime}} .
$$

This immediately gives us the desired inequality.

\section{UnWeighted $L^{2}$ Kato estimates}

In this section we prove unweighted $L^{2}$ estimates for the operators we have considered in the previous sections. These will all be consequences of the weighted $L^{p}(v d w)$ estimates we have already proved: it will only be necessary to find further conditions on $w \in A_{2}$ so that the weight $v=w^{-1}$ satisfies the requisite conditions.

We are particularly interested in power weights and we recall some well-known facts about them. Define $w_{\alpha}(x)=|x|^{\alpha}, \alpha>-n$; this restriction guarantees that $w_{\alpha}$ is locally integrable. We can exactly determine the Muckenhoupt $A_{p}$ and reverse Hölder $R H_{s}$ classes of these weights in terms of $\alpha$ : if $-n<\alpha \leq 0$, then $w \in A_{1}$; for $1<p<\infty, w \in A_{p}$ if $-n<\alpha<n(p-1)$. Furthermore, if $0 \leq \alpha<\infty, w \in R H_{\infty}$; for $1<q<\infty, w \in R H_{q}$. if $-n / q<\alpha<\infty$. Hence, we easily see that

$$
r_{w_{\alpha}}=\max \{1,1+\alpha / n\}, \quad s_{w_{\alpha}}=\left(\max \left\{1,(1+\alpha / n)^{-1}\right\}\right)^{\prime} .
$$


We first consider the semigroup $e^{-t L_{w}}$, the functional calculus, and the square function $g_{L_{w}}$, since these estimates will depend on $p_{-}\left(L_{w}\right)$ and $p_{+}\left(L_{w}\right)$ and we have good estimates for these quantities.

Theorem 11.2. Given a weight $w \in A_{2}$, suppose $1 \leq r_{w}<1+\frac{2}{n}$ and $s_{w}>\frac{n}{2} r_{w}+1$. Then $e^{-t L_{w}}: L^{2} \rightarrow L^{2}$ is uniformly bounded for all $t>0$. Similarly, $\varphi\left(L_{w}\right): L^{2} \rightarrow L^{2}$, where $\varphi$ is any bounded holomorphic function on $\Sigma_{\mu}, \mu \in(\vartheta, \pi)$, and $g_{L_{w}}: L^{2} \rightarrow L^{2}$.

In particular, these $L^{2}$ estimates hold if we assume that $w \in A_{1} \cap R H_{1+\frac{n}{2}}$, or more generally if $w \in A_{r} \cap R H_{\frac{n}{2} r+1}$ for $1<r \leq 1+\frac{2}{n}$, or if we take the power weights

$$
w_{\alpha}(x)=|x|^{\alpha}, \quad-\frac{2 n}{n+2}<\alpha<2 .
$$

Proof. Let $p=q=2, p_{0}=\left(2_{w}^{*}\right)^{\prime}, q_{0}=2_{w}^{*}$, and let $v=w^{-1}$. Then by Proposition 3.1, Corollary 3.3 and the nesting properties of weights, $e^{-t L_{w}} \in \mathcal{O}\left(L^{2} \rightarrow L^{2}\right)$ if $w^{-1} \in A_{2 / p_{0}}(w) \cap$ $R H_{\left(q_{0} / 2\right)^{\prime}}(w)$; in particular, by Lemma $2.30, e^{-t L_{w}}: L^{2} \rightarrow L^{2}$ is uniformly bounded. However, this weight condition is equivalent to

$$
w \in R H_{\left(2 / p_{0}\right)^{\prime}} \cap A_{q_{0} / 2} .
$$

A straightforward computation shows that

$$
\frac{q_{0}}{2}=\frac{n r_{w}}{n r_{w}-2}, \quad\left(\frac{2}{p_{0}}\right)^{\prime}=\frac{n}{2} r_{w}+1 .
$$

Since $r_{w}<1+\frac{2}{n}$, we have that $r_{w}<\frac{n r_{w}}{n r_{w}-2}$, so we automatically have that $w \in A_{q_{0} / 2}$. Therefore, the desired bounds hold if we have $s_{w}>\frac{n}{2} r_{w}+1$. If $w \in A_{r} \cap R H_{\frac{n}{2} r+1}$ with $1 \leq r \leq 1+\frac{2}{n}$, then $r_{w} \leq r$ and $s_{w}>\frac{n}{2} r+1 \geq \frac{n}{2} r_{w}+1$. The desired conclusion for power weights follows at once from (11.1).

The same argument holds for $\varphi\left(L_{w}\right)$ and $g_{L_{w}}$, using Proposition 4.3 or Proposition 5.1, respectively.

It is straightforward to construct weights more general than power weights that satisfy the conditions on $r_{w}$ and $s_{w}$ in the above theorems. For instance, $w \in A_{1+\frac{2}{n}} \cap R H_{2+\frac{n}{2}}$ (which corresponds to the choice $r=1+\frac{2}{n}$ ) if and only if there exist $u_{1}, u_{2} \in A_{1}$ such that

$$
w=u_{1}^{\frac{2}{n+4}} u_{2}^{-\frac{2}{n}} \text {. }
$$

This follows from the Jones factorization theorem and the properties of $A_{1}$ weights: cf. [15].

Remark 11.3. We can modify the proof of Theorem 11.2 to get unweighted $L^{p}$ estimates for values of $p$ close to 2 . We leave the details to the interested reader.

For the reverse inequalities we need to take into account the slightly stronger hypotheses in Proposition 6.1; otherwise, the proof of the following result follows exactly as in the proof of Theorem 11.2.

Theorem 11.4. Given a weight $w \in A_{2}$, suppose that

Then

$$
1 \leq r_{w}<1+\frac{2}{n} \quad \text { and } s_{w}>\max \left\{\left(\frac{2}{r_{w}}\right)^{\prime}, \frac{n}{2} r_{w}+1\right\} \text {. }
$$

$$
\left\|L_{w}^{1 / 2} f\right\|_{L^{2}} \leq C\|\nabla f\|_{L^{2}}, \quad f \in \mathcal{S}
$$


In particular, this is the case if we either assume that $w \in A_{1} \cap R H_{1+\frac{n}{2}}$, or more generally that $w \in A_{r} \cap R H_{\max \left\{\left(\frac{2}{r}\right)^{\prime}, \frac{n}{2} r+1\right\}}$, with $1<r \leq 1+\frac{2}{n}$, or for power weights if we take

$$
w_{\alpha}(x)=|x|^{\alpha}, \quad-\frac{2 n}{n+2}=-\min \left\{\frac{n}{2}, \frac{2 n}{n+2}\right\}<\alpha<2 .
$$

Remark 11.6. Note that $\max \left\{\left(\frac{2}{r}\right)^{\prime}, \frac{n}{2} r+1\right\}=\frac{n}{2} r+1$ provided $r \leq 2-\frac{2}{n}$ and this always holds if $n \geq 4$ as $1+\frac{2}{n} \leq 2-\frac{2}{n}$. In this case, the conditions in the second part of Theorem 11.4 simplify to the same conditions as in Theorem 11.2.

Remark 11.7. We note that in Theorems 11.2 and 11.4 we can replace $1 \leq r_{w}<1+\frac{2}{n}$ with the possibly weaker condition $1 \leq r_{w}<\frac{p_{+}\left(L_{w}\right)}{2}$. The proof only requires us to take $q_{0}=p_{+}\left(L_{w}\right)$.

For the gradient of the semigroup $\sqrt{t} \nabla e^{-t L_{w}}$, the Riesz transform $\nabla L_{w}^{-1 / 2}$, and the square function $G_{L_{w}}$, our estimates depend on $q_{+}\left(L_{w}\right)$.

Theorem 11.8. Given a weight $w \in A_{2}$, suppose $1 \leq r_{w}<\frac{q_{+}\left(L_{w}\right)}{2}$ and $s_{w}>\frac{n}{2} r_{w}+1$. Then $\sqrt{t} \nabla e^{-t L_{w}}: L^{2} \rightarrow L^{2}$ is uniformly bounded for all $t>0$. Similarly, we have that $\nabla L_{w}^{-1 / 2}: L^{2} \rightarrow L^{2}$ and $G_{L_{w}}: L^{2} \rightarrow L^{2}$.

In particular, this is the case if we assume that $w \in A_{1} \cap R H_{\frac{n}{2}+1}$. Furthermore, these $L^{2}$ estimates hold if the following is true: given $\Theta \geq 1$ there exists $\epsilon_{0}=\epsilon_{0}(\Theta, n, \Lambda / \lambda)$, $0<\epsilon_{0} \leq \frac{1}{2 n}$, such that $w \in A_{1+\epsilon} \cap R H_{\frac{n}{2}(1+\epsilon)+1}, 0 \leq \epsilon<\epsilon_{0}$, and $[w]_{A_{2}} \leq \Theta$. for

For power weights, there exists $\epsilon_{1}=\epsilon_{1}(n, \Lambda / \lambda), 0<\epsilon_{1} \leq \frac{1}{2}$, such that these estimate holds

$$
w_{\alpha}(x)=|x|^{\alpha}, \quad-\frac{2 n}{n+2}<\alpha<\epsilon_{1} .
$$

Proof. We will prove this result for $\sqrt{t} \nabla e^{-t L_{w}}$ using Proposition 7.1. The proof for $\nabla L_{w}^{-1 / 2}$ or $G_{L_{w}}$ is exactly the same, using Proposition 9.1 or Proposition 10.1.

By Proposition 7.1, $\sqrt{t} \nabla e^{-t L_{w}}: L^{2} \rightarrow L^{2}$ if $w^{-1}=v \in A_{2 / q_{-}\left(L_{w}\right)}(w) \cap R H_{\left.q_{+}\left(L_{w}\right) / 2\right)^{\prime}}(w)$, which is equivalent to

$$
w \in R H_{\left(2 / q_{-}\left(L_{w}\right)\right)^{\prime}} \cap A_{q_{+}\left(L_{w}\right) / 2} .
$$

Therefore, we need $r_{w}<q_{+}\left(L_{w}\right) / 2$. Furthermore, since we have that $q_{-}\left(L_{w}\right)=p_{-}\left(L_{w}\right) \leq$ $\left(2_{w}^{*}\right)^{\prime}$, we can take

$$
s_{w}>\left(\frac{2}{\left(2_{w}^{*}\right)^{\prime}}\right)^{\prime}=\frac{n}{2} r_{w}+1 .
$$

To get the particular examples stated in the theorem, note first that if we let $r_{w}=1$, then it clearly suffices to assume $w \in A_{1} \cap R H_{\frac{n}{2}+1}$, since we showed in Section 8 that $q_{+}\left(L_{w}\right)>2$ for every $w \in A_{2}$.

We now prove the condition for weights $w \in A_{1+\epsilon}$. In this case it is more difficult to satisfy the condition $r_{w}<q_{+}\left(L_{w}\right) / 2$ since the righthand side can be very close to 1 depending on $w$. Assume then that $w \in A_{1+\epsilon} \cap R H_{\frac{n}{2}(1+\epsilon)+1}$, with $0 \leq \epsilon<\epsilon_{0} \leq \frac{1}{2 n},[w]_{A_{2}} \leq \Theta$, and with $\epsilon_{0}>0$ to be fixed below. Then we have that

$$
s_{w}>\frac{n}{2}(1+\epsilon)+1 \geq \frac{n}{2} r_{w}+1
$$


Therefore, in order to apply the first half of the theorem we need to show that we can choose $\epsilon_{0}$ sufficiently small so that $r_{w}<q_{+}\left(L_{w}\right) / 2$. To do so we will use the notation and computations from Section 8. There we showed that $q_{+}\left(L_{w}\right) \geq q_{w}$, and so it will suffice to show that

$$
2 r_{w}<q_{w}=\min \left(r_{w}^{\prime}, p_{+}\left(L_{w}\right), p_{0}\right) .
$$

We will compare $r_{w}$ to each term in the minimum in turn.

The first two terms are straightforward. First, we have that $r_{w}<1+\epsilon<1+\frac{1}{2 n}<\frac{3}{2}$ and so $2 r_{w}<r_{w}^{\prime}$. Second, $r_{w}<1+\frac{1}{2 n}<1+\frac{2}{n}$, and it follows at once from this that $2 r_{w}<2_{w}^{*}$. By Proposition 3.1, $2_{w}^{*} \leq p_{+}\left(L_{w}\right)$ and so $2 r_{w}<p_{+}\left(L_{w}\right)$.

Finally, we estimate $p_{0}$, the exponent from the higher integrability condition (8.3). We will use the formula (8.4). First, we need to fix the exponent $q$ from the Poincaré inequality (8.2). Let $q=2-1 / n$; this value satisfies (8.1) since $r_{w}<1+\frac{1}{2 n}<1+\frac{1}{n}$. With this choice of $q$ (that only depends on $n$ ), we have that

$$
p_{0}=2+\frac{2-q}{2^{4 / q+1} C_{1}^{2} C_{2}^{2}[w]_{A_{2}}^{6 / q+17}}=2+\frac{1}{n C(n, \Lambda / \lambda)[w]_{A_{2}}^{\theta_{n}}}
$$

where $C(n, \Lambda / \lambda) \geq 1$ depends only on $n$ and the ratio $\Lambda / \lambda$ of the ellipticity constants of the matrix $A$ used to define $L_{w}$, and where $\theta_{n} \geq 1$ depends only on $n$. Then, since we also assumed that $[w]_{A_{2}} \leq \Theta$, we get that

$$
p_{0}=2+\frac{1}{n C(n, \Lambda / \lambda)[w]_{A_{2}}^{\theta_{n}}} \geq 2+\frac{1}{n C(n, \Lambda / \lambda) \Theta^{\theta_{n}}}=2+2 \epsilon_{0},
$$

and $\epsilon_{0}=\left(2 n C(n, \Lambda / \lambda) \Theta^{\theta_{n}}\right)^{-1}$ is such that $0<\epsilon_{0} \leq \frac{1}{2 n}$. Thus $2 r_{w}<2(1+\epsilon)<2\left(1+\epsilon_{0}\right) \leq$ $p_{0}$ and so $2 r_{w}<p_{0}$. This completes the proof that (11.9) is satisfied, and so the $L^{2}$ estimates hold for weights that satisfy $w \in A_{1+\epsilon} \cap R H_{\frac{n}{2}(1+\epsilon)+1}$.

Finally, we consider power weights. First, it is easy to see that

$$
w_{\alpha}(x)=|x|^{\alpha}, \quad \frac{-2 n}{n+2}<\alpha \leq 0
$$

yields the desired estimates, since in this case $r_{w}=1$ and $s_{w}>\frac{n}{2}+1=\frac{n}{2} r_{w}+1$.

Now consider the case $\alpha>0$. If we assume that $\alpha<\frac{1}{2}$, then $w \in A_{1+\frac{1}{2 n}} \cap R H_{\infty}$. Moreover, it is straightforward to show that for all such $\alpha$, there exists $\Theta$, depending only on $n$, such that $\left[w_{\alpha}\right]_{A_{2}} \leq \Theta$. Now apply the above argument to find $\epsilon_{0} \in\left(0, \frac{1}{2 n}\right]$; this value will only depend on $n$ and the ratio $\Lambda / \lambda$. If we let $\epsilon_{1}=n \epsilon_{0}$ and assume that $0<\alpha<\epsilon_{1}$, then $\alpha<\frac{1}{2}$ and $w_{\alpha} \in A_{1+\epsilon}$ for some $\epsilon<\epsilon_{0}$ as desired.

To find examples of weights other than power weights to which Theorem 11.8 apply, we argue as before. If $u_{1} \in A_{1}$, then

$$
w=u_{1}^{\frac{2}{n+2}} \in A_{1} \cap R H_{\frac{n}{2}+1} .
$$

To get weights that are not in $A_{1}$, take $u \in A_{2}$ and let $w=u^{\theta}$. If $\theta$ is sufficiently small (depending on $n$, the ratio $\Lambda / \lambda$ and $[u]_{A_{2}}$ ) we can show that $w$ satisfies the final conditions given in Theorem 11.8. Details are left to the interested reader. 
Remark 11.10. To get the unweighted lower estimate

$$
\|f\|_{L^{2}} \leq C\left\|G_{L_{w}} f\right\|_{L^{2}}
$$

we note that by $(10.6)$ we need $w^{-1} \in A_{2 / q_{+}\left(\Delta_{w}\right)^{\prime}}(w)$, or equivalently, $w \in R H_{\left(2 / q_{+}\left(\Delta_{w}\right)^{\prime}\right)^{\prime}}$. Hence, it suffices to assume

$$
s_{w}>1+\frac{q_{+}\left(\Delta_{w}\right)}{q_{+}\left(\Delta_{w}\right)-2} .
$$

Arguing as above we can construct weights that satisfy this condition; details are left to the interested reader.

If we combine Theorems 11.4, 11.8, and Remark 11.7 we solve the Kato square root problem for degenerate elliptic operators.

Theorem 11.11. Let $L_{w}=-w^{-1} \operatorname{div} A \nabla$ be a degenerate elliptic operator with $w \in A_{2}$. If

$$
1 \leq r_{w}<\frac{q_{+}\left(L_{w}\right)}{2} \quad \text { and } \quad s_{w}>\max \left\{\left(\frac{2}{r_{w}}\right)^{\prime}, \frac{n}{2} r_{w}+1\right\} .
$$

then the Kato problem can be solved for $L_{w}$ : that is, for every $f \in H^{1}\left(\mathbb{R}^{n}\right)$.

$$
\left\|L_{w}^{1 / 2} f\right\|_{L^{2}\left(\mathbb{R}^{n}\right)} \approx\|\nabla f\|_{L^{2}\left(\mathbb{R}^{n}\right)}
$$

where the implicit constants depend only on the dimension, the ellipticity constants $\lambda, \Lambda$, and $w$.

In particular, (11.12) holds if $w \in A_{1} \cap R H_{\frac{n}{2}+1}$. Further, (11.12) holds if the following is true: given $\Theta \geq 1$ there exists $\epsilon_{0}=\epsilon_{0}(\Theta, n, \Lambda / \lambda), 0<\epsilon_{0} \leq \frac{1}{2 n}$, such that $w \in A_{1+\epsilon} \cap$ $R H_{\max \left\{\left(\frac{2}{1+\epsilon}\right)^{\prime}, \frac{n}{2}(1+\epsilon)+1\right\}}, 0 \leq \epsilon<\epsilon_{0}$, and $[w]_{A_{2}} \leq \Theta$.

For power weights, there exists $\epsilon_{1}=\epsilon_{1}(n, \Lambda / \lambda), 0<\epsilon_{1} \leq \frac{1}{2}$, such that inequality (11.12) holds (with $w_{\alpha}$ in place of $w$ ) if

$$
w_{\alpha}(x)=|x|^{\alpha}, \quad-\frac{2 n}{n+2}<\alpha<\epsilon_{1} .
$$

We can restate the final part of Theorem 11.11 as follows: consider the family of operators $L_{\gamma}=-|x|^{\gamma} \operatorname{div}\left(|x|^{-\gamma} B(x) \nabla\right)$, where $B$ is an $n \times n$ complex-valued matrix that satisfies the uniform ellipticity condition

$$
\lambda|\xi|^{2} \leq \operatorname{Re}\langle B(x) \xi, \xi\rangle, \quad|\langle B(x) \xi, \eta\rangle| \leq \Lambda|\xi||\eta|, \quad \xi, \eta \in \mathbb{C}^{n} \text {, a.e. } x \in \mathbb{R}^{n} .
$$

Then,

$$
\left\|L_{\gamma}^{1 / 2} f\right\|_{L^{2}\left(\mathbb{R}^{n}\right)} \approx\|\nabla f\|_{L^{2}\left(\mathbb{R}^{n}\right)}, \quad-\epsilon_{1}<\gamma<\frac{2 n}{n+2} .
$$

When $\gamma=0$ we get the classical Kato square root problem solved by Auscher, Hofmann, Lacey, McIntosh, and Tchamitchian [4]. Inequality (11.13) shows that we can find an open interval containing 0 such that if $\gamma$ is in this interval, the same estimate holds. 


\section{Applications to $L^{2}$ Boundary value problems}

In this section we apply the results from the previous section to some $L^{2}$ boundary value problems involving the degenerate elliptic operator $L_{w}$. We follow the ideas in [10] and consider semigroup solutions: for the Dirichlet or Regularity problems we let $u(x, t)=$ $e^{-t L_{w}^{1 / 2}} f(x)$; for the Neumann problem we let $u(x, t)=-L_{w}^{-1 / 2} e^{-t L_{w}^{1 / 2}} f(x)$. In each case, for $t>0$ fixed $L_{w} u(\cdot, t)$ makes sense in a weak sense since $u(\cdot, t)$ is in the domain of $L_{w}$. Further, derivatives in $t$ are well defined because of the semigroup properties. Finally, note that by the strong continuity of the semigroup and the off-diagonal estimates, in the context of the following results we have that $e^{-t L_{w}^{1 / 2}} f \rightarrow f$ as $t \rightarrow 0^{+}$in $L^{2}$; see [7, Section 4.2]. Further details are left to the interested reader.

We first consider the Dirichlet problem on $\mathbb{R}_{+}^{n+1}=\mathbb{R}^{n} \times[0, \infty)$ :

$$
\begin{cases}\partial_{t}^{2} u-L_{w} u=0, & \text { on } \mathbb{R}^{n} \\ \left.u\right|_{\partial \mathbb{R}_{+}^{n+1}}=f & \text { on } \partial \mathbb{R}_{+}^{n+1}=\mathbb{R}^{n}\end{cases}
$$

Theorem 12.2. Given a weight $w \in A_{2}$, suppose $1 \leq r_{w}<1+\frac{2}{n}$ and $s_{w}>\frac{n}{2} r_{w}+1$. Then for any $f \in L^{2}\left(\mathbb{R}^{n}\right), u(x, t)=e^{-t L_{w}^{1 / 2}} f(x)$ is a solution of $(12.1)$ with convergence to the boundary data as $t \rightarrow 0^{+}$in the $L^{2}$-sense. Furthermore, we have that

$$
\sup _{t>0}\|u(\cdot, t)\|_{L^{2}} \leq C\|f\|_{L^{2}}
$$

In particular, this is the case if we assume that $w \in A_{1} \cap R H_{1+\frac{n}{2}}$, or $w \in A_{r} \cap R H_{\frac{n}{2} r+1}$ with $1<r \leq 1+\frac{2}{n}$, or if we take the power weights

$$
w_{\alpha}(x)=|x|^{\alpha}, \quad-\frac{2 n}{n+2}<\alpha<2 .
$$

Proof. Formally, it is clear that $u$ is a solution to (12.1), and this formalism can be justified by appealing to the theory of maximal accretive operators: see Kato [25]. Alternatively, the weighted estimates for the functional calculus in Proposition 4.3 show that both $\frac{\partial^{2}}{\partial t^{2}} u(\cdot, t)$ and $L_{w} u(\cdot, t)$ belong to $L^{2}$ for each $t>0$ and that they are equal in the $L^{2}$-sense. To see that inequality $(12.3)$ holds, it suffices to let $\varphi_{t}(z)=e^{-t \sqrt{z}}$. Then $\varphi_{t}$ is a bounded holomorphic function on $\Sigma_{\mu}$, and so by Theorem 11.2 we get the desired bound.

Remark 12.4. Note that as observed in Remark 11.7, in the previous result we can replace $1 \leq r_{w}<1+\frac{2}{n}$ with the possibly weaker condition $1 \leq r_{w}<\frac{p_{+}\left(L_{w}\right)}{2}$. Also, by Proposition 4.3 we also have that for $u$ as in Theorem 12.2 and all $k \geq 1$

$$
\sup _{t>0}\left\|t^{k} \frac{\partial^{k}}{\partial t^{k}} u(\cdot, t)\right\|_{L^{2}}=\sup _{t>0}\left\|\left(t^{k} L_{w}^{1 / 2}\right)^{k} e^{-t L_{w}^{1 / 2}} f(\cdot)\right\|_{L^{2}} \leq C\|f\|_{L^{2}} .
$$

For the regularity problem we have the following.

Theorem 12.6. Given a weight $w \in A_{2}$, suppose

$$
1 \leq r_{w}<\frac{q_{+}\left(L_{w}\right)}{2} \quad \text { and } \quad s_{w}>\max \left\{\left(\frac{2}{r_{w}}\right)^{\prime}, \frac{n}{2} r_{w}+1\right\} .
$$


Then for any $f \in H^{1}\left(\mathbb{R}^{n}\right), u(x, t)=e^{-t L_{w}^{1 / 2}} f(x)$ is a solution of (12.1) with convergence to the boundary data as $t \rightarrow 0^{+}$in the $L^{2}$-sense. Furthermore, we have that

$$
\sup _{t>0}\left\|\nabla_{x, t} u(\cdot, t)\right\|_{L^{2}} \leq C\|\nabla f\|_{L^{2}} .
$$

In particular, (12.7) holds if we assume that $w \in A_{1} \cap R H_{1+\frac{n}{2}}$. Furthermore, it holds if the following is true: given $\Theta \geq 1$ there exists $\epsilon_{0}=\epsilon_{0}(\Theta, n, \Lambda / \lambda), 0<\epsilon_{0} \leq \frac{1}{2 n}$, such that $w \in A_{1+\epsilon} \cap R H_{\max \left\{\left(\frac{2}{1+\epsilon}\right)^{\prime}, \frac{n}{2}(1+\epsilon)+1\right\}}, 0 \leq \epsilon<\epsilon_{0}$, and $[w]_{A_{2}} \leq \Theta$.

For power weights, there exists $\epsilon_{1}=\epsilon_{1}(n, \Lambda / \lambda), 0<\epsilon_{1} \leq \frac{1}{2}$, such that (12.7) holds if

$$
w_{\alpha}(x)=|x|^{\alpha}, \quad-\frac{n}{2}<\alpha<\epsilon_{1} .
$$

Proof. Arguing as before, it suffices to prove that (12.7) holds. For any $t>0$ we have, by Theorem 11.11

$$
\begin{aligned}
\left\|\nabla_{x, t} u(\cdot, t)\right\|_{L^{2}} \leq \| \nabla L_{w}^{-1 / 2} & L_{w}^{1 / 2} e^{-t L_{w}^{1 / 2}} f\left\|_{L^{2}}+\right\| L_{w}^{1 / 2} e^{-t L_{w}^{1 / 2}} f \|_{L^{2}} \\
& \lesssim\left\|L_{w}^{1 / 2} e^{-t L_{w}^{1 / 2}} f\right\|_{L^{2}}=\left\|e^{-t L_{w}^{1 / 2}} L_{w}^{1 / 2} f\right\|_{L^{2}} \lesssim\left\|L_{w}^{1 / 2} f\right\|_{L^{2}} \lesssim\|\nabla f\|_{L^{2}} .
\end{aligned}
$$

Note that under the hypothesis of Theorem 12.6, and as observed in Remark 12.4, we have that $u(\cdot, t)=e^{-t L_{w}^{1 / 2}} f$ satisfies (12.3) and (12.5). Additionally, from the functional calculus estimates on $L^{2}$ it follows that

$$
\sup _{t>0}\left\|t \nabla_{x, t} u(\cdot, t)\right\|_{L^{2}} \lesssim\left\|t L_{w}^{1 / 2} e^{-t L_{w}^{1 / 2}} f\right\|_{L^{2}} \lesssim\|f\|_{L^{2}}
$$

Finally, we consider the Neumann problem

$$
\begin{cases}\partial_{t}^{2} u-L_{w} u=0, & \text { on } \mathbb{R}^{n} \\ \left.\partial_{t} u\right|_{\partial \mathbb{R}_{+}^{n+1}}=f & \text { on } \partial \mathbb{R}_{+}^{n+1}=\mathbb{R}^{n} .\end{cases}
$$

Theorem 12.10. Given a weight $w \in A_{2}$, suppose $1 \leq r_{w}<\frac{q_{+}\left(L_{w}\right)}{2}$ and $s_{w}>\frac{n}{2} r_{w}+1$. Then for any $f \in L^{2}\left(\mathbb{R}^{n}\right), u(x, t)=-L_{w}^{-1 / 2} e^{-t L_{w}^{1 / 2}} f(x)$ is a solution of (12.9) with convergence of $\partial_{t} u(\cdot, t) \rightarrow f$ as $t \rightarrow 0^{+}$in the $L^{2}$-sense. Furthermore, we have that

$$
\sup _{t>0}\left\|\nabla_{x, t} u(\cdot, t)\right\|_{L^{2}} \leq C\|f\|_{L^{2}} .
$$

In particular, (12.11) holds if we assume that $w \in A_{1} \cap R H_{1+\frac{n}{2}}$. Furthermore, it holds if the following is true: given $\Theta \geq 1$ there exists $\epsilon_{0}=\epsilon_{0}(\Theta, n, \Lambda / \lambda), 0<\epsilon_{0} \leq \frac{1}{2 n}$, such that $w \in A_{1+\epsilon} \cap R H_{\frac{n}{2}(1+\epsilon)+1}, 0 \leq \epsilon<\epsilon_{0}$, and $[w]_{A_{2}} \leq \Theta$.

For power weights, there exists $\epsilon_{1}=\epsilon_{1}(n, \Lambda / \lambda), 0<\epsilon_{1} \leq \frac{1}{2}$, such that (12.11) holds if

$$
w_{\alpha}(x)=|x|^{\alpha}, \quad-\frac{2 n}{n+2}<\alpha<\epsilon_{1} .
$$

Proof. Again, $u$ is clearly a formal solution of (12.9); see [25]. The proof that (12.11) holds is similar to the proof of (12.7):

$$
\left\|\nabla_{x, t} u(\cdot, t)\right\|_{L^{2}} \leq\left\|\nabla L_{w}^{-1 / 2} e^{-t L_{w}^{1 / 2}} f\right\|_{L^{2}}+\left\|e^{-t L_{w}^{1 / 2}} f\right\|_{L^{2}} \lesssim\left\|e^{-t L_{w}^{1 / 2}} f\right\|_{L^{2}} \lesssim\|f\|_{L^{2}},
$$

where we have used Theorem 11.8 (for the Riesz transform) and Theorem 11.2 (for the functional calculus with $\left.\varphi(z)=e^{-t \sqrt{z}}\right)$. 
Remark 12.12. As we noted in Remark 11.3, we can also get unweighted $L^{p}$ bounds for these operators for values of $p$ close to 2 . As a consequence we can also get estimates $L^{p}$ boundary value problems for the same values of $p$. Details are left to the reader.

\section{REFERENCES}

[1] T. Alberico, A. Cianchi, and C. Sbordone. Fractional integrals and $A_{p}$-weights: a sharp estimate. $C$. R. Math. Acad. Sci. Paris, 347(21-22):1265-1270, 2009.

[2] P. Auscher. On necessary and sufficient conditions for $L^{p}$-estimates of Riesz transforms associated to elliptic operators on $\mathbb{R}^{n}$ and related estimates. Mem. Amer. Math. Soc., 186(871):xviii+75, 2007.

[3] P. Auscher and T. Coulhon. Riesz transform on manifolds and Poincaré inequalities. Ann. Sc. Norm. Super. Pisa Cl. Sci. (5), 4(3):531-555, 2005.

[4] P. Auscher, S. Hofmann, M. Lacey, A. McIntosh, and P. Tchamitchian. The solution of the Kato square root problem for second order elliptic operators on $\mathbb{R}^{n}$. Ann. of Math. (2), 156(2):633-654, 2002.

[5] P. Auscher and J. M. Martell. Weighted norm inequalities, off-diagonal estimates and elliptic operators. III. Harmonic analysis of elliptic operators. J. Funct. Anal., 241(2):703-746, 2006.

[6] P. Auscher and J. M. Martell. Weighted norm inequalities, off-diagonal estimates and elliptic operators. I. General operator theory and weights. Adv. Math., 212(1):225-276, 2007.

[7] P. Auscher and J. M. Martell. Weighted norm inequalities, off-diagonal estimates and elliptic operators. II. Off-diagonal estimates on spaces of homogeneous type. J. Evol. Equ., 7(2):265-316, 2007.

[8] P. Auscher and J. M. Martell. Weighted norm inequalities, off-diagonal estimates and elliptic operators. IV. Riesz transforms on manifolds and weights. Math. Z., 260(3):527-539, 2008.

[9] P. Auscher, A. Rosén, and D. Rule. Boundary value problems for degenerate elliptic equations and systems. Ann. Sci. Éc. Norm. Supér. (4), 48(4):951-1000, 2015.

[10] P. Auscher and P. Tchamitchian. Square root problem for divergence operators and related topics. Astérisque, (249):viii+172, 1998.

[11] F. Bernicot and J. Zhao. New abstract Hardy spaces. J. Funct. Anal., 255(7):1761-1796, 2008.

[12] A. Björn and J. Björn. Nonlinear potential theory on metric spaces, volume 17 of EMS Tracts in Mathematics. European Mathematical Society (EMS), Zürich, 2011.

[13] L. Chen, J. M. Martell, and C. Prisuelos-Arribas. Conical square functions for degenerate elliptic operators. To appear in Adv. Calc. Var., 2017. arXiv:1610.05952.

[14] M. Cowling, I. Doust, A. McIntosh, and A. Yagi. Banach space operators with a bounded $H^{\infty}$ functional calculus. J. Austral. Math. Soc. Ser. A, 60(1):51-89, 1996.

[15] D. Cruz-Uribe and C. J. Neugebauer. The structure of the reverse Hölder classes. Trans. Amer. Math. Soc., 347(8):2941-2960, 1995.

[16] D. Cruz-Uribe and C. Rios. Gaussian bounds for degenerate parabolic equations. J. Funct. Anal., 255(2):283-312, 2008.

[17] D. Cruz-Uribe and C. Rios. The solution of the Kato problem for degenerate elliptic operators with Gaussian bounds. Trans. Amer. Math. Soc., 364(7):3449-3478, 2012.

[18] D. Cruz-Uribe and C. Rios. Corrigendum to "Gaussian bounds for degenerate parabolic equations" [J. Funct. Anal. 255 (2) (2008) 283-312] [mr2419963]. J. Funct. Anal., 267(9):3507-3513, 2014.

[19] D. Cruz-Uribe and C. Rios. The Kato problem for operators with weighted ellipticity. Trans. Amer. Math. Soc., 367(7):4727-4756, 2015.

[20] J. Duoandikoetxea. Fourier analysis, volume 29 of Graduate Studies in Mathematics. American Mathematical Society, Providence, RI, 2001.

[21] E. B. Fabes, C. Kenig, and R. Serapioni. The local regularity of solutions of degenerate elliptic equations. Comm. Partial Differential Equations, 7(1):77-116, 1982.

[22] J. García-Cuerva and J. L. Rubio de Francia. Weighted norm inequalities and related topics, volume 116 of North-Holland Mathematics Studies. North-Holland Publishing Co., Amsterdam, 1985.

[23] M. Haase. The functional calculus for sectorial operators, volume 169 of Operator Theory: Advances and Applications. Birkhäuser Verlag, Basel, 2006.

[24] S. Hofmann, P. Le, and A. Morris. Carleson measure estimates and the Dirichlet problem for degenerate elliptic equations. Preprint, 2015. 
[25] T. Kato. Perturbation theory for linear operators. Die Grundlehren der mathematischen Wissenschaften, Band 132. Springer-Verlag New York, Inc., New York, 1966.

[26] P. Le. $L^{p}$ bounds of Riesz transform and vertical square functions for degenerate elliptic operators. Preprint, 2015.

[27] A. McIntosh. Operators which have an $H_{\infty}$ functional calculus. In Miniconference on operator theory and partial differential equations (North Ryde, 1986), volume 14 of Proc. Centre Math. Anal. Austral. Nat. Univ., pages 210-231. Austral. Nat. Univ., Canberra, 1986.

[28] N. Miller. Weighted Sobolev spaces and pseudodifferential operators with smooth symbols. Trans. Amer. Math. Soc., 269(1):91-109, 1982.

[29] C. Pérez. Calderón-Zygmund theory related to Poincaré-Sobolev inequalities, fractional integrals and singular integral operators. In J. Lukes and L. Pick, editors, Function spaces, nonlinear analysis and applications, Lectures notes of the spring lectures in analysis. Charles University and Academy of Sciences, 1999. Available at http://grupo.us.es/anaresba/trabajos/carlosperez/31.pdf.

[30] D. Yang and J. Zhang. Weighted $L^{p}$ estimates of Kato quare roots associated to degenerate elliptic operators. Publ. Mat., 61(2):395-444, 2017.

David Cruz-Uribe, OfS, Department of Mathematics, University of Alabama, Tuscaloosa, AL 35487-0350, USA

E-mail address: dcruzuribe@ua.edu

José María Martell, Instituto de Ciencias Matemáticas CSiC-UAM-UC3M-UCM, Consejo Superior de Investigaciones Científicas, C/ Nicolás Cabrera, 13-15, E-28049 Madrid, Spain E-mail address: chema.martell@icmat.es

AND

Department of Mathematics, University of Missouri, Columbia, MO 65211, USA

E-mail address: martellj@missouri.edu

Cristian Rios, Department of Mathematics and Statistics, University of Calgary, 2500

University Drive NW, Calgary, AB, T2N-1N4, Canada

E-mail address: crios@ucalgary.ca 